\title{
Compressible, Multiphase Semi-Implicit Method with Moment of Fluid Interface Representation *
}

\author{
Matthew Jemison, Mark Sussman \\ Department of Applied \& Computational Mathematics \\ Florida State University \\ Marco Arienti \\ Sandia National Laboratories
}

September 6, 2014

\begin{abstract}
A unified method for simulating multiphase flows using an exactly mass, momentum, and energy conserving Cell-Integrated SemiLagrangian advection algorithm is presented. The deforming material boundaries are represented using the moment-of-fluid method. The new algorithm uses a semi-implicit pressure update scheme that asymptotically preserves the standard incompressible pressure projection method in the limit of infinite sound speed. The asymptotically preserving attribute makes the new method applicable to compressible and incompressible flows including stiff materials; enabling large time

${ }^{*}$ Work supported in part by the National Science Foundation under contract DMS 1016381. M. Arienti acknowledges the support by Sandia National Laboratories via the Laboratory Directed Research and Development program. Sandia National Laboratories is a multi-program laboratory managed and operated by Sandia Corporation, a wholly owned subsidiary of Lockheed Martin Corporation, for the U. S. Department of Energy's National Nuclear Security Administration under contract DE-AC04-94AL85000. The technical assistance from M. Shashkov regarding the multimaterial moment-of-fluid method is
\end{abstract} great-fully acknowledged. 
steps characteristic of incompressible flow algorithms rather than the small time steps required by explicit methods. Shocks are captured and material discontinuities are tracked, without the aid of any approximate or exact Riemann solvers. Simulations of underwater explosions and fluid jetting in one, two, and three dimensions are presented which illustrate the effectiveness of the new algorithm at efficiently computing multiphase flows containing shock waves and material discontinuities with large "impedance mismatch."

\section{Introduction}

There are many applications in science and industry that necessitate the understanding of compressible, multiphase flows. Examples include underwater explosions and implosions $[55,54,17,32]$, bubble dynamics $[44,35,11,1,46]$, shock wave lithotripsy [42, 30], atomization and spray in internal-combustion engines [4, 9], aerobreakup [51], and laser induced melting [61, 43].

Since the development of the Implicit Continuous-Fluid Eulerian (ICE) method [23] in the late 1960's, there have been many new algorithms developed for simulating compressible multiphase flow $[23,18,54,44,35,60,41$, $32,39,19,9,22,17,11,1,12]$. A summary of the key properties of algorithms that have been developed for simulating compressible multiphase flows are given chronologically in Table 1. 


\begin{tabular}{|c|c|c|c|c|}
\hline Authors & $\begin{array}{c}\text { Interface } \\
\text { Representation }\end{array}$ & $\begin{array}{c}\text { Interface } \\
\text { Treatment }\end{array}$ & $\begin{array}{c}\text { Mass of Each } \\
\text { Material Conserved }\end{array}$ & $\begin{array}{c}\text { Asymptotically } \\
\text { Preserving }\end{array}$ \\
\hline $\begin{array}{l}\text { Harlow and } \\
\text { Amsden[23] }\end{array}$ & $\begin{array}{c}\text { Lagrangian } \\
\text { Particles }\end{array}$ & $\begin{array}{l}\text { Finite Difference } \\
\text { Method }\end{array}$ & No & Yes \\
\hline $\begin{array}{l}\text { Fedkiw, } \\
\text { et. al. }[18]\end{array}$ & Level-Set & $\begin{array}{l}\text { Ghost Fluid } \\
\text { Method }\end{array}$ & No & No \\
\hline $\begin{array}{l}\text { Wardlaw and } \\
\text { Mair [54] }\end{array}$ & $\begin{array}{c}\text { Arbitrary Lagrangian } \\
\text { Eulerian+Remap }\end{array}$ & Front-Tracking & Yes & No \\
\hline $\begin{array}{l}\text { Saurel and } \\
\text { Abgrall [44] }\end{array}$ & Diffuse Interface & $\begin{array}{c}\text { Riemann Solver/ } \\
\text { Godunov }\end{array}$ & No & No \\
\hline $\begin{array}{l}\text { Koren, } \\
\text { et. al. }[35]\end{array}$ & Level Set & $\begin{array}{c}\text { Finite Volume, } \\
\text { Ghost Fluid }\end{array}$ & No & No \\
\hline $\begin{array}{l}\text { Yabe, } \\
\text { et. al. }[60]\end{array}$ & Diffuse Interface & $\begin{array}{l}\text { Cubic Interpolated } \\
\text { Profile }\end{array}$ & No & Yes \\
\hline $\begin{array}{l}\text { Nourgaliev, } \\
\text { et. al. }[41]\end{array}$ & Level Set & $\begin{array}{l}\text { Characteristics } \\
\text { Based Matching }\end{array}$ & No & No \\
\hline $\begin{array}{l}\text { Kadioglu, } \\
\text { et. al. }[32]\end{array}$ & Level Set & $\begin{array}{l}\text { Ghost Fluid } \\
\text { Method }\end{array}$ & No & Yes \\
\hline $\begin{array}{l}\text { Galera, } \\
\text { et.al. }[19]\end{array}$ & Moment-of-Fluid & $\begin{array}{c}\text { Arbitrary Lagrangian } \\
\text { Eulerian }\end{array}$ & Yes & No \\
\hline $\begin{array}{l}\text { Bo, } \\
\text { et. al. }[9]\end{array}$ & Front Tracking & $\begin{array}{l}\text { Ghost Fluid } \\
\text { Method }\end{array}$ & No & No \\
\hline $\begin{array}{l}\text { Gretarsson, } \\
\text { et. al. }[22]\end{array}$ & Front Tracking & $\begin{array}{l}\text { Ghost Fluid } \\
\text { Method }\end{array}$ & Yes & Yes \\
\hline $\begin{array}{l}\text { Farhat, } \\
\text { et. al. }[17]\end{array}$ & $\begin{array}{c}\text { Level Set, Two Phase } \\
\text { Riemann Solver }\end{array}$ & $\begin{array}{c}\text { Finite Volume - } \\
\text { Ghost Fluid Method }\end{array}$ & No & No \\
\hline $\begin{array}{l}\text { Chang, } \\
\text { et. al. [11] }\end{array}$ & Level Set & $\begin{array}{l}\text { Riemann Solver/ } \\
\text { Finite Volume }\end{array}$ & Yes & No \\
\hline $\begin{array}{l}\text { Gretarsson and } \\
\text { Fedkiw [21] }\end{array}$ & Front Tracking & $\begin{array}{l}\text { Ghost Fluid } \\
\text { Method }\end{array}$ & Yes & Yes \\
\hline $\begin{array}{l}\text { Aanjaneya, } \\
\text { et. al. }[1]\end{array}$ & Level Set & $\begin{array}{l}\text { Ghost Fluid } \\
\text { Method }\end{array}$ & No & Yes \\
\hline $\begin{array}{l}\text { Cheng and } \\
\text { Shu [12] }\end{array}$ & Lagrangian & $\begin{array}{l}\text { positivity preserving, } \\
\text { approx. Riemann solve }\end{array}$ & Yes & No \\
\hline
\end{tabular}

Table 1: Chronological listing of methods in compressible multiphase flows.

Asymptotic preservation is defined in the sense of Degond, et. al. [14]. If the method is expressed as a perturbation in terms of the sound speed, such that in the limit as sound speed $c^{2} \rightarrow \infty$, the incompressible pressure projection method is recovered, then the method is said to be "asymptotically preserving."

It is demonstrated in articles as early as $1968[23,60,32,31,22,21,1]$ 
that numerical algorithms for multiphase flow that have the asymptotically preserving property are robust for computing solutions in which materials with widely varying properties neighbor each other. This is because the "monolithic" semi-implicit discretization for pressure leads to strong coupling between all the materials. For example we validate our asymptotically preserving method in section 6.8 for the buoyancy driven motion of a gas bubble in water in which both the gas and water are governed by realistic equations of state and realistic physical properties. We are not aware of a non-asymptotically preserving method that can compute this problem. Other important properties of our asymptotically preserving algorithm are that our algorithm discretely enforces the divergence free constraint $\nabla \cdot \boldsymbol{u}=0$ in the infinite sound speed limit $c \rightarrow \infty$ (i.e. our method is a volume preserving method in the infinite sound speed limit), enables larger time steps, and eliminates the need for customized Riemann solvers in order to track the speed of discontinuities.

Often explicit numerical methods for computing compressible multiphase flows implement a Riemann solver in order to determine the velocity of material interfaces. The Riemann solver finds the interface velocity necessary so that the pressure and normal velocity on either side of a material interface is continuous at the new time step. In our formulation, we solve for the new pressure and velocity by solving the Helmholtz equation for pressure; solutions to the Helmholtz equation are sufficiently smooth so that the new pressure and advective velocity will be continuous across different materials. As we shall describe later in the paper, we apply a geometric constant contact condition when interpolating pressure from cell centers to cell faces which ensures that the new cell velocity is also continuous across material interfaces at each time step.

Besides having the asymptotically preserving property, our method is cast locally in conservation form in order to accurately compute the correct speed of shock waves[11, 19, 54]. We find (see section 6.1) that our asymptotically preserving algorithm captures shock waves with comparable accuracy as modern high order shock capturing schemes.

We note from Table 1 that few compressible multiphase flow methods are asymptotically preserving. Those that are asymptotically preserving may not conserve mass of each material individually, or may cast the problem in non-conservative form.

There have been a number of recent single-phase methods that exhibit the asymptotic preserving property in the limit of infinite sound speed $[56,58$, 
$59,40,36,6]$. The method introduced by Kwatra et al[36] stands out in that they split, in a stable fashion, the Euler equations for gas-dynamics into an advection part and pressure correction part without sacrificing conservation or the asymptotically preserving property.

In our new algorithm, we are hybridizing the recent multimaterial, conservative, semi-Lagrangian developments presented by Galera et. al. [20, 19] with the asymptotic preserving developments recently introduced by Kwatra et. al. [36] in order to produce a novel method that simultaneously captures correct shock speed and magnitude, sharply tracks material discontinuities without the aid of Riemann solvers or mixed material pressure formulations, and performs robustly in compressible and nearly incompressible flow regimes. In our new algorithm, material discontinuities are represented by the moment-of-fluid method $[2,16]$ making our algorithm well-suited to deformational, multi-material flows.

We remark that Gretarsson et al[22, 21] describe conservative, asymptotically preserving algorithms for fluid-structure interaction. Our work is different in that we implemented the Cell Integrated Semi-Lagrangian (CISL) approach for discretizing the advective terms and we represent deforming boundaries by the moment-of-fluid method. We have taken the CISL/Moment-of-Fluid approach because this approach should enable us to extend our present algorithm to multiphase flows with any number of materials by making use of the volume preserving multimaterial moment-of-fluid interface reconstruction algorithm[2, 16].

Our paper is organized as follows: Section 2 defines the governing equations, Section 3 presents the Multiphase Cell Integrated Semi-Lagrangian (CISL) advection scheme, Section 3.1 discusses the Moment-of-Fluid interface reconstruction algorithm, Section 4 discusses the multiphase pressure correction scheme, Section 5 discusses the scheme used in order to stably trace backwards along characteristics, and Section 6 presents various benchmark tests and novel numerical results. 


\section{Governing Equations}

We are solving the Navier-Stokes equations for multimaterial flows in which there are $M$ materials, $m=1, \ldots M$.

$$
\left(\begin{array}{c}
F^{m} \\
\rho^{m} F^{m} \\
\rho \boldsymbol{u} \\
\rho^{m} E^{m} F^{m}
\end{array}\right)_{t}+\nabla \cdot\left(\begin{array}{c}
F^{m} \boldsymbol{u} \\
\rho^{m} F^{m} \boldsymbol{u} \\
\boldsymbol{u} \otimes \rho \boldsymbol{u}+p \mathbb{I}-\tau(\boldsymbol{u}) \\
\left(\left(\rho^{m} E^{m}+p\right) \boldsymbol{u}-\boldsymbol{u} \cdot \tau(\boldsymbol{u})-k \nabla T\right) F^{m}
\end{array}\right)=\left(\begin{array}{c}
F^{m} \nabla \cdot \boldsymbol{u} \\
0 \\
\mathbf{F}_{b o d y} \\
\boldsymbol{u} \cdot \mathbf{F}_{\text {body }} F^{m}
\end{array}\right)
$$

$F^{m}$ is the volume fraction of material $m, \rho^{m}$ is the density of material $m$, $\rho^{m} E^{m}$ is the energy of material $m$, and $\boldsymbol{u}$ is the velocity. Tensor $\tau$ represents the stress tensor (2.2):

$$
\tau(\boldsymbol{u})=2 \mu\left(D-\frac{\mathrm{TR}(D) \mathbb{I}}{D I M}\right) \quad D=\frac{(\nabla \boldsymbol{u})+(\nabla \boldsymbol{u})^{T}}{2} \quad D I M=2 \text { or } 3
$$

Force $\mathbf{F}_{\text {body }}$ represents the body force. The thermal diffusivity, $k$, is taken to be zero for this work.

The combined density is,

$$
\rho=\sum_{m=1}^{M} \rho^{m} F^{m}
$$

Here, energy $E^{m}$ and internal energy $e_{i n t}^{m}$ are related such that Eqn. 2.3 holds.

$$
\rho^{m} E^{m}=\rho^{m} e_{i n t}^{m}+\frac{1}{2} \rho^{m}|\boldsymbol{u}|^{2}
$$

\section{Directionally-Split Multiphase Advection}

Following the work of Kwatra et al [36], we split the solution of (2.1) into two parts: (1) advection and (2) semi-implicit pressure correction for momentum and energy.

In contrast to Kwatra et al [36], we have developed a directionally split Cell Integrated Semi-Lagrangian algorithm (CISL) for advection. Our CISL algorithm, coupled with moment-of-fluid interface reconstruction, enables sharp preservation of material interfaces, conservation of mass for each material, and conservation of overall momentum and energy. We use CISL 
advection for multimaterial flows rather than alternative methods for its inherent conservation of flow variables, straightforward manner of computing multiphase flows, and robustness in the presence of deformational flows $[20,19]$.

Directionally split advection is performed using either a backtracing or combined Eulerian-Implicit/Lagrangian-Explicit (EI-LE) [45] advection scheme.

\subsection{Interface Reconstruction}

Materials are separated by an interface $\Gamma$, which is represented with a piecewiselinear interface reconstruction in each cell, $\Gamma_{i}$. After advection, a cell may contain multiple materials. If this is the case, the location of the interface must be reconstructed. Multiple methods exist for this procedure. The Level Set Method is one such technique that tracks the location of an interface via a signed distance function, with the zero contour denoting the material interface. However, the method does not preserve volume in highly deformational flows [8].

The Volume of Fluid Method corrects this problem by reconstructing a material interface such that the volume fraction of material in the departure region is equal to the reconstructed volume fraction. The drawback of volume-of-fluid style methods is that non-local data must be considered in determining the orientation of the interface. In two dimensions, this may involve all points in the surrounding $3 \times 3$ stencil, depending on the method used [8]. Additionally, reconstruction of more than two materials relies on the "onion skin" model. In the moment-of-fluid method, the first interface $\Gamma_{1}$ in cell $i$ denotes the boundary for material $\Omega_{i}^{1}$. The second interface $\Gamma_{2}$ is reconstructed such that materials $\Omega_{i}^{1}$ and $\Omega_{i}^{2}$ are contained in the region. In general, $\Gamma_{m}$ is reconstructed such that material regions $\Omega_{i}^{1}$ through $\Omega_{i}^{m}$ are contained in the cut region. The moment-of-fluid technique allows for the possibility of intersecting material interfaces. We note that the moment of fluid reconstruction algorithm, while capable of reconstructing exactly multimaterial planar intersecting interfaces in an element, fails to reconstruct most triple point configurations.

The Moment of Fluid Method tracks volume fractions (3.1) through advection, as does the volume of fluid method, but uses the centroid (3.2) of a material region (i.e. the first moment) in determining the orientation of the interface [16]. The interface is chosen as the piece-wise linear reconstruction, $\Gamma_{i}^{m}(3.3)$, that exactly captures the volume fraction and minimizes error in 
the centroid. Centroid error can be interpreted as curvature in the interface, which can be used as a condition for mesh refinement in AMR algorithms $[3]$.

$$
\begin{gathered}
F_{i}^{m}=\frac{\left|\Omega_{i}^{m}\right|}{\left|\Omega_{i}\right|} \\
\boldsymbol{x}_{i}^{m}=\frac{\int_{\Omega_{i}^{m}} \boldsymbol{x} d \Omega}{\left|\Omega_{i}^{m}\right|} \\
\Gamma_{i}^{m}=\left\{\boldsymbol{x} \mid \hat{n}_{i}^{m} \cdot\left(\boldsymbol{x}-\boldsymbol{x}_{i}\right)+b_{i}^{m}=0 \text { and } \boldsymbol{x} \in \Omega_{i}\right\}
\end{gathered}
$$

Using the directionally split backtracing method in Section 3.3, a departure volume for a cell is calculated using face velocities. Material in the departure region is mapped into the cell of interest, undergoing expansion or compression based on the derivative of the face velocity. Reference volume fractions $\left(F_{r e f}\right)$ and reference centroids $\left(\boldsymbol{x}_{r e f}\right)$ are then calculated in the cell of interest. An interface $\Gamma(\hat{n}, b)$ cutting the cell will yield an actual volume fraction $\left(F_{a c t}\right)$ and actual centroid $\left(\boldsymbol{x}_{a c t}\right)$, which are functions of the parameters $\hat{n}$ and $b$. The optimal interface will satisfy (3.4):

$$
(\hat{n}, b)=\underset{\hat{n}, b,\left|F_{a c t}(\hat{n}, b)-F_{r e f}\right|=0}{\arg \min }\left|\boldsymbol{x}_{a c t}(\hat{n}, b)-\boldsymbol{x}_{r e f}\right|
$$

To limit numerical errors, volume fractions within a cut-off tolerance of zero or one are truncated as in (3.5). The tolerance for our multidimensional test problems was taken to be $\epsilon=10^{-8}$. Also the volume fractions are normalized to add to one (3.6).

$$
\begin{gathered}
F^{m} \leftarrow \begin{cases}0, & F^{m}<\epsilon \\
F^{m}, & \epsilon<F^{m}<1-\epsilon \quad m=1, \ldots, M \\
1, & F^{m}>1-\epsilon\end{cases} \\
F=\sum_{m=1}^{M} F^{m} \\
F^{m} \leftarrow \frac{F^{m}}{F}
\end{gathered}
$$

In $1 D$, results using the moment of fluid method and volume of fluid method will be identical. However, in higher dimension, the moment of fluid 
method has been shown to out perform the volume of fluid method on a number of benchmark tests [16], [29]. In problems with more than two materials, the moment-of-fluid method has the ability to capture a triple point, as well as more complex material configurations, and has been extended to perform reconstruction in the presence of an arbitrary number of materials, without overlapping interfaces [2]. Robustness and accuracy in the presence of multiple materials can be improved by the Symmetric Moment-of-Fluid interface reconstruction algorithm [25], which uses the moment information of both the extracted and remaining material to find the optimal interface configuration.

\subsection{Reconstruction of conserved variables $\rho, \rho u$ and $\rho E$.}

In each cell, $\Omega_{i}$, and for each material $m$, we are given the density, momentum, and energy,

$$
\boldsymbol{U}_{i}^{m} \equiv\left(\rho_{i}^{m}, \rho_{i}^{m} \boldsymbol{u}_{i}, \rho_{i}^{m} E_{i}^{m}\right)
$$

$\rho_{i}^{m}$ and $E_{i}^{m}$ are given at material $m$ 's centroid within the cell $\Omega_{i}: \boldsymbol{x}_{i}^{m} . \boldsymbol{x}_{i}^{m}$ is defined in (3.2). The velocity $\boldsymbol{u}_{i}$ is given at the centroid of cell $\Omega_{i}, \boldsymbol{x}_{i}$, which is defined as (3.8):

$$
\boldsymbol{x}_{i}=\frac{\int_{\Omega_{i}} \boldsymbol{x} d \Omega}{\left|\Omega_{i}\right|} .
$$

In our method, we define the reconstruction of $\boldsymbol{U}_{i}^{m}$ as a piecewise linear reconstruction. In order that our Semi-Lagrangian transport scheme exactly preserve conservation of mass, momentum, and energy, we require that the piecewise linear reconstruction of conserved quantities have the following form:

$$
\mathcal{U}_{i}^{m}(x)=\left(\boldsymbol{U}^{\prime}\right)_{i}^{m}\left(x-x_{i}^{m}\right)+\boldsymbol{U}_{i}^{m} .
$$

The slope in (3.9) is defined to be the MINMOD slope; the MINMOD slope for the linear reconstruction of a scalar variable is (3.10):

$$
U_{i}^{\prime}=\left\{\begin{array}{cc}
0 & \text { if }\left(U_{i+1}-U_{i}\right)\left(U_{i}-U_{i-1}\right) \leq 0 \\
\frac{U_{i+1}-U_{i}}{\Delta x} & \text { if }\left|U_{i+1}-U_{i}\right|<\left|U_{i}-U_{i-1}\right| \\
\frac{U_{i}-y_{i-1}}{\Delta x} & \text { otherwise }
\end{array}\right.
$$


To perform backtracing advection, the departure region $\Omega_{i}^{D}$ for a cell $\Omega_{i}$ is found. The departure region will intersect $\Omega_{i}$ and can intersect neighboring cells, $\left\{\Omega_{i^{\prime}}\right\}$. Figure 1 illustrates the reconstruction and CISL advection of density. If density, momentum, and energy have a piecewise linear reconstruction, $\mathcal{U}_{i}^{m}(x)$ (3.9), and the time step is chosen such that no departure region collapses to have zero volume, then the following $1 \mathrm{D}$ advection scheme preserves overall mass, momentum, and energy:

$$
\begin{array}{r}
F_{i}^{m, n+1}=\frac{\sum_{i^{\prime}} \int_{\Omega_{i}^{D} \cap \Omega_{i^{\prime}}} H_{i^{\prime}}^{m, n, R}(x) d \Omega}{\sum_{i^{\prime}}\left|\Omega_{i}^{D} \cap \Omega_{i^{\prime}}\right|} \\
\boldsymbol{U}_{i}^{m, n+1}=\frac{\sum_{i^{\prime} \Omega_{i}^{D} \cap \Omega_{i^{\prime}}} H_{i^{\prime}}^{m, n, R}(x) \mathcal{U}_{i^{\prime}}^{m, n}(x) d \Omega}{F_{i}^{m, n+1}\left|\Omega_{i}\right|}
\end{array}
$$

$H_{i}^{m, n, R}(x)$ is a material indicator function for the moment of fluid reconstructed interface in cell $i$. Referring to (3.3), we have,

$$
H_{i}^{m, n, R}(x)=\left\{\begin{array}{cc}
1 & \hat{n}_{i}^{m} \cdot\left(\boldsymbol{x}-\boldsymbol{x}_{i}\right)+b_{i}^{m} \geq 0 \text { and } \boldsymbol{x} \in \Omega_{i} \\
0 & \text { otherwise }
\end{array}\right.
$$

Remarks:

- For a cell cut by a material interface, $F_{i}^{m}<1$ or $F_{i \pm 1}^{m}=0$, we prescribe the material $m$ slopes in cell $\Omega_{i}$ to be zero: $\left(\boldsymbol{U}^{\prime}\right)_{i}^{m}=0$.

- We have compared results when the slope for the reconstruction of conserved variables is always prescribed to be zero versus when the slopes are defined to be the MINMOD slope. See Figures 24 and 25. We do not see much of a difference in the results so that in all of our $2 \mathrm{D}$ or $3 \mathrm{D}$ simulations, we prescribe the reconstructed slopes to always be zero. 

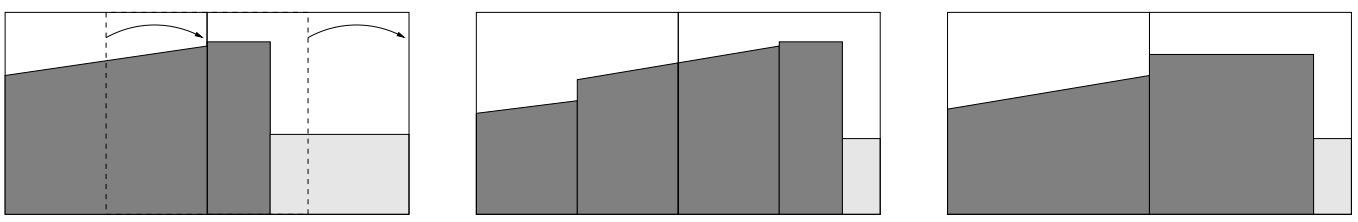

Figure 1: Illustration of 1D CISL advection of density $\rho$. Material from the departure region is advected to the target cell. The state variable and slope are then reconstructed in the target cell to conserve mass in each material. The dark and light shaded regions represent separate, immiscible materials.

\subsection{Lagrangian Characteristic Tracing}

In one dimension, we have implemented a backwards characteristic tracing cell integrated semi-Lagrangian algorithm. See Figure 2. In two and three dimensions, we have implemented a directionally split cell integrated semiLagrangian algorithm in which we alternate between the backwards characteristic tracing algorithm and the forward characteristic tracing algorithm. In $2 \mathrm{D}$, the advection algorithm follows the following pattern,

$\begin{array}{cc}R \text { sweep at } t^{n} & \text { Eulerian Implicit } \\ Z \text { sweep at } t^{n} & \text { Lagrangian Explicit } \\ Z \text { sweep at } t^{n+1} & \text { Eulerian Implicit } \\ R \text { sweep at } t^{n+1} & \text { Lagrangian Explicit } \\ R \text { sweep at } t^{n+2} & \text { Eulerian Implicit ... }\end{array}$

The backward characteristic tracing algorithm is referred to as "Eulerian Implicit" (EI) and the forward characteristic tracing algorithm is referred to as "Lagrangian Explicit" (LE). Eulerian Implicit (EI) refers to backward tracing advection which maps material from a departure region. Lagrangian Explicit (LE) refers to forward tracing advection, which maps material to a target region. We refer the reader to $[45,7]$ for the motivation of why we have implemented the alternating EI and LE directionally split method for simulating 2D and 3D multiphase flows.

We first describe the Eulerian Implicit (backwards tracing of characteristics) algorithm illustrated in Figure 2. In $1 \mathrm{D}$ coordinates, a cell $\Omega_{i}$ is defined as follows:

$$
\Omega_{i}=\left[x_{i-1 / 2}, x_{i+1 / 2}\right] .
$$


The size of $\Omega_{i}$ is

$$
\Delta x \equiv\left|\Omega_{i}\right|=x_{i+1 / 2}-x_{i-1 / 2} .
$$

We define the departure region $\Omega_{i}^{D}$ for cell $\Omega_{i}(3.17)$ as the solution to the characteristic ODE (3.16) at time $t=\Delta t$, with the initial position $x_{\text {face }}$ taken at cell faces, and face velocity $u_{\text {face }}$.

$$
\left\{\begin{array}{l}
\frac{d x}{d t}=-u_{\text {face }} \\
x(t=0)=x_{\text {face }}
\end{array}\right.
$$

Departure region $\Omega_{i}^{D}$ represents a forward Euler approximation of the region that will be mapped to cell $i$ under advection.

$$
\Omega_{i}^{D}=\left[x_{i-1 / 2}-\Delta t u_{i-1 / 2}, x_{i+1 / 2}-\Delta t u_{i+1 / 2}\right]
$$

Points in the departure region are then mapped to the cell of interest, or "target cell," under a linear mapping (3.18).

$$
\begin{aligned}
f_{i}(x) & =\mathcal{C}\left(x-\left(x_{i-1 / 2}-\Delta t u_{i-1 / 2}\right)\right)+x_{i-1 / 2} \\
\mathcal{C} & =\frac{\left|\Omega_{i}\right|}{\left|\Omega_{i}^{D}\right|}=\frac{1}{1-\frac{\Delta t}{\Delta x}\left(u_{i+1 / 2}-u_{i-1 / 2}\right)}
\end{aligned}
$$

The characteristic tracing method coupled with the mapping (3.18) from the departure region to the target cell will cause compression/expansion when the factor $\mathcal{C} \neq 1$.

We now describe the Lagrangian Explicit (forward tracing of characteristics) algorithm. For the forward sweeping advection strategy, material is distributed from the departure cell to a "target region," $\Omega_{i}^{T}(3.19)$.

$$
\Omega_{i}^{T}=\left[x_{i-1 / 2}+\Delta t u_{i-1 / 2}, x_{i+1 / 2}+\Delta t u_{i+1 / 2}\right]
$$

We refer the reader to Figure 3 for an illustration of the two dimensional, EI-LE, directionally split cell integrated semi-Lagrangian method. 


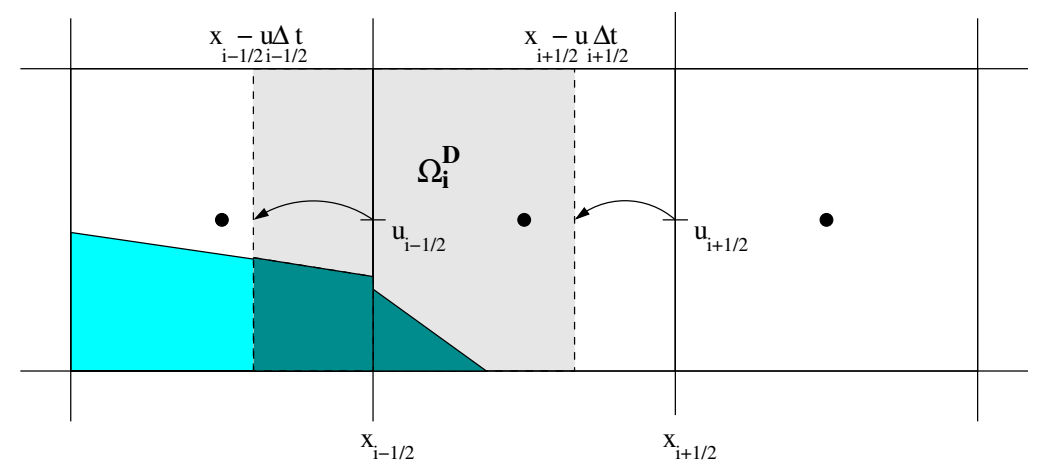

Figure 2: Characteristic backtracing, given face velocity $u_{i \pm 1 / 2}$. Departure region $\Omega_{i}^{D}$ for cell "i" is shown as the shaded region. A material region $\Omega^{m}$ is shown in light blue. The intersection of the material with the departure region $\Omega^{m} \cap \Omega_{i}^{D}$ is shown as a darker blue.
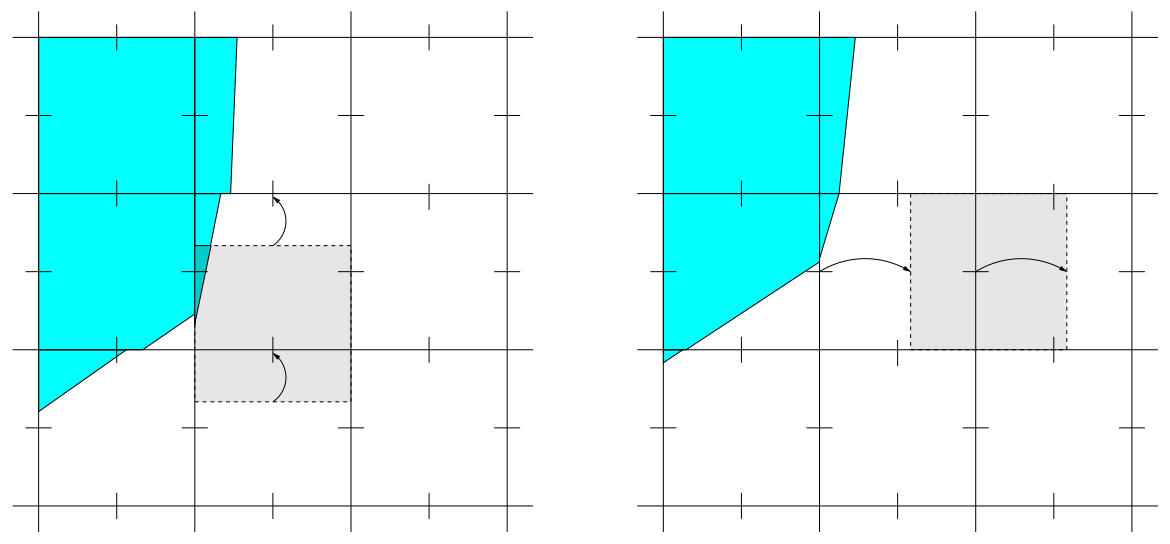

Figure 3: Euler-Implicit/Lagrangian-Explicit directionally split, two sweep scheme in 2D. Velocity is located at face centroids, shown as a dash. Left: Backtracing is performed on the first sweep, and the interface is reconstructed. Right: Forward tracing is performed on the reconstructed interface for the second sweep. Departure region is shown as the shaded region. Material $m$ is shown as blue.

\subsection{CISL Multiphase Advection}

Without loss of generality, we describe the backwards tracing, Eulerian Implicit, CISL Multiphase Advection algorithm. We refer the reader to Table 2 for the definitions of variables and symbols used in our description. Sup- 
pose that the domain contains $M$ immiscible materials, referred to with a superscript $m$. Discretely, assuming zero net flux across the boundaries, conservation of mass, momentum, and energy equates to satisfying (3.20) in each material $m$, for reconstruction $\mathcal{U}_{i}^{n}(x)$ in cell $\Omega_{i}$ at time $t_{n}$.

$$
\sum_{i=0}^{N} \int_{\Omega_{i}^{m, n}} \mathcal{U}_{i}^{m, n}(x) d \Omega=\sum_{i=0}^{N} \int_{\Omega_{i}^{m, 0}} \mathcal{U}_{i}^{m, 0}(x) d \Omega
$$

The region of material occupied by material $m$ in a cell $i$ is denoted by $\Omega_{i}^{m}$, so a cell may be written as the union of these material regions (3.21).

$$
\Omega_{j}=\bigcup_{m=1}^{M} \Omega_{j}^{m}
$$

When departure region $\Omega_{i}^{D}$ (3.17) is intersected with the mesh, it must be independently intersected with each material region. Assuming that the materials stay in constant contact, we may write a cell $\Omega_{j}$ as the union of material domains within that cell (3.21). If at least two regions $\Omega_{j}^{m_{1}}$ and $\Omega_{j}^{m_{2}}$ are non-empty, then cell $\Omega_{j}$ is referred to as a "cut cell."

This informs how to perform advection with multiple materials. A material configuration in each cell is given at time $t_{n}$. Volume fractions are advected, and a moment-of-fluid interface reconstruction is performed as described in Section 3.1. This defines the reconstructed material regions for the cell of interest, $\Omega_{j}$ at time $t_{n+1}$.

1. Advect and Update volume fractions and moments for each material, $m$. Volume fractions and centroid are computed after mapping material forward, for correct moment computation in Cartesian and curvilinear coordinates.

$$
\begin{array}{r}
F_{j}^{m, n+1}=\frac{\sum_{i^{\prime}}\left|\Omega_{i^{\prime}}^{m} \cap \Omega_{j}^{D}\right|}{\sum_{i^{\prime}}\left|\Omega_{i^{\prime}} \cap \Omega_{j}^{D}\right|} \\
\boldsymbol{x}_{j}^{m, n+1}=\frac{\sum_{i^{\prime}} \int_{\Omega_{i^{\prime}, j}^{m, T} \cap \Omega_{j}} \boldsymbol{x} d \Omega}{\sum_{i^{\prime}}\left|\Omega_{i^{\prime}, j}^{m, T} \cap \Omega_{j}\right|}
\end{array}
$$

$\Omega_{i^{\prime}, j}^{m, T}$ is the image of $\Omega_{i^{\prime}, j}^{m}$ under the action of $f_{j}$ (3.18). In other words,

$$
f_{j}: \Omega_{i, j}^{m} \rightarrow \Omega_{i, j}^{m, T}
$$




\begin{tabular}{|c|c|}
\hline$\Omega_{j}^{m}$ & Region of cell $\Omega_{j}$ occupied by material "m" \\
\hline$\Omega_{j}^{D}$ & $\begin{array}{c}\text { Lagrangian departure region (see Section 3.3) } \\
\text { for cell } \Omega_{j}\end{array}$ \\
\hline$\Omega_{i^{\prime}, i}^{m, T}$ & $\begin{array}{l}\text { The image of region "m" in cell } \Omega_{i^{\prime}} \text { under the linear } \\
\text { mapping } f_{i} \text { from the departure region } \Omega_{i}^{D} \text { to cell } \Omega_{i}\end{array}$ \\
\hline$F_{j}^{m, n+}$ & $\begin{array}{l}\text { Fraction of material "m" in cell } \Omega_{j} \text { after } \\
\text { mapping from the departure region }\end{array}$ \\
\hline $\boldsymbol{x}_{j}^{m, n+1}$ & $\begin{array}{l}\text { Centroid of the region occupied by material "m" in } \Omega_{j} \text { after } \\
\text { all material is mapped from the departure region }\end{array}$ \\
\hline $\boldsymbol{U}_{j}^{m, n}$ & $\begin{array}{c}\text { Conserved variables, } \boldsymbol{U}_{j}^{m, n} \equiv\left(\rho_{j}^{m, n},(\rho \boldsymbol{u})_{j}^{m, n},(\rho E)_{j}^{m, n}\right), \\
\text { of material "m" in cell } \Omega_{j} \text { at time } t^{n} .\end{array}$ \\
\hline $\mathcal{U}_{j}^{m, n}(x)$ & $\begin{array}{c}\text { Linear reconstruction (3.9) of conserved variables for } \\
\text { material "m" in cell } \Omega_{j} \text { at time } t^{n} \text {. }\end{array}$ \\
\hline $\boldsymbol{u}_{j}^{a}$ & $\begin{array}{l}\text { Time advanced CISL solution to }(\rho \boldsymbol{u})_{t}+\nabla \cdot(\rho \boldsymbol{u} \otimes \boldsymbol{u})=0 \\
\text { for velocity in cell } \Omega_{j}\end{array}$ \\
\hline$E_{j}^{m, a}$ & $\begin{array}{l}\text { Time advanced CISL solution to }\left(\rho E^{m}\right)_{t}+\nabla \cdot\left(\rho \boldsymbol{u} E^{m}\right)=0 \\
\text { for specific energy for material } m \text { in cell } \Omega_{j} .\end{array}$ \\
\hline$\rho_{j}^{m *}$ & $\begin{array}{c}\text { Density of the material occupying the maximum } \\
\text { volume in cell } \Omega_{j}\end{array}$ \\
\hline
\end{tabular}

Table 2: Key for notation in the Cell-Integrated Semi-Lagrangian (CISL) advection section. In general, sub-scripts refer to locations on the grid, and super-scripts refer to material ID or time level.

2. Advect and Update conserved variables, $\boldsymbol{U}=(\rho, \rho \boldsymbol{u}, \rho E)$, for Each Material, $m$

$$
\boldsymbol{U}_{j}^{m, n+1}=\frac{\sum_{i^{\prime}} \int_{\Omega_{i^{\prime}}^{m} \cap \Omega_{j}^{D}} \mathcal{U}_{i^{\prime}}^{m, n}(x) d \Omega}{\left|\Omega_{j}^{m, n+1}\right|}
$$

3. Derive the advected velocity $\boldsymbol{u}_{j}^{a}$ from the conserved variables

$$
\boldsymbol{u}_{j}^{a}=\frac{\sum_{m=1}^{M}\left|\Omega_{j}^{m, n+1}\right|(\rho \boldsymbol{u})_{j}^{m, n+1}}{\sum_{m=1}^{M}\left|\Omega_{j}^{m, n+1}\right|(\rho)_{j}^{m, n+1}}
$$


4. Derive the advected Total Energy $E_{j}^{m, a}$ and advected Internal Energy for Each Material, $m$

$$
\begin{gathered}
E_{j}^{m, a}=\frac{(\rho E)_{j}^{m, n+1}}{(\rho)_{j}^{m, n+1}} \\
e_{i n t, j}^{m, a}=E_{j}^{m, a}-1 / 2\left|\boldsymbol{u}_{j}^{a}\right|^{2}
\end{gathered}
$$

5. The "advective pressure," $p^{a}$ (3.28), and the "advective sound speed," $\left(c^{2}\right)^{a}(3.29)$, are defined using the equation of state as a function of the advective internal energy and advective density. The pressure in a cut cell is taken as the equation of state evaluated for the material $m^{*}$ that occupies the largest volume fraction in the cell. Pressure is not a conserved quantity, so it is neither necessary nor physically relevant to use a conservative Semi-Lagrangian advection strategy to find $p^{a}$. Work by Gretarsson and Fedkiw [21] indicates that use of the equation of state (EOS) reduces oscillation at a discontinuity, when compared with a third-order accurate Hamilton-Jacobi ENO scheme for finding the advective pressure.

$$
\begin{gathered}
p_{j}^{a}=\operatorname{EOS}^{m^{*}}\left(e_{i n t}^{m^{*}, a}, \rho^{m^{*}, n+1}\right) \\
c^{2}=\left(\frac{\partial p\left(\rho, e_{i n t}\right)}{\partial \rho}\right)_{e_{i n t}}+\frac{p}{\rho^{2}}\left(\frac{\partial p\left(\rho, e_{i n t}\right)}{\partial e_{i n t}}\right)_{\rho} \\
\left(c^{2}\right)^{a}=c^{2}\left(e_{i n t}^{m^{*}, a}, \rho^{m^{*}, n+1}\right)
\end{gathered}
$$

Remark: The following conditions for the backwards tracing of characteristics CISL advection algorithm enable our method to conserve mass for each material and conserve overall momentum and energy:

(a) The reconstructed materials in any given computational cell $\Omega_{i}$ form a tessellation of the cell and the reconstructed volume equals the reference volume:

$$
\Omega_{i}=\cup_{m=1}^{M} \Omega_{i}^{m}, \quad \Omega_{i}^{m_{1}} \cap \Omega_{i}^{m_{2}}=\emptyset \quad m_{1} \neq m_{2} \quad\left|\Omega_{i}^{m}\right|=|\Omega| F_{i}^{m}
$$

(b) The reconstruction of conserved variables for each material $m$ satisfies,

$$
\int_{\Omega_{i}^{m}} \mathcal{U}_{i}^{m}(\boldsymbol{x}) d \boldsymbol{x}=\boldsymbol{U}_{i}^{m}\left|\Omega_{i}^{m}\right| .
$$


(c) The mapping functions $f_{i}$ derived from the face velocity must be defined so that the regions $\Omega_{i}^{D} \cap \Omega_{i^{\prime}}^{m}$ form a tessellation of the computational domain where $i^{\prime}=i-1, i, i+1, m=1, \ldots, M$, and $i$ ranges over all the computational cells.

(d) The image of all the materially intersected regions, $\Omega_{i} \cap \Omega_{i^{\prime}, i}^{m, T}$, also form a tessellation of the computational domain where $i^{\prime}=i-1, i, i+1$, $m=1, \ldots, M$, and $i$ ranges over all the computational cells.

We impose a linear mapping and piecewise linear interface reconstruction which guarantees that materially intersected regions and their images are polyhedral regions with straight edges. For future work, a higher order CISL advection algorithm can be developed in which an unsplit advection algorithm (instead of directionally split) is used, the interface reconstruction is piecewise quadratic and the mapping functions are piecewise quadratic. The key is that conditions (a)-(d) are satisfied.

\section{Multiphase, Semi-Implicit Pressure Correc- tion}

The action of advection is separated from the pressure force terms in the Euler equations as in [36]. Our numerical algorithm first finds the advected quantities, velocity $\boldsymbol{u}^{a}$, density $\rho^{n+1}$, and total energy $E^{a}$ using the CISL advection algorithm described in section (3.4). Then our numerical method defines the advective pressure $p^{a}$ (3.28), advective internal energy, $e^{a}$ (3.27), and advective sound speed $\left(c^{2}\right)^{a}(3.29)$ as follows:

$$
\begin{array}{r}
e^{a}=E^{a}-\frac{1}{2} \boldsymbol{u}^{a} \cdot \boldsymbol{u}^{a} \\
p^{a}=p\left(\rho^{n+1}, e^{a}\right) \\
\left(c^{2}\right)^{a}=c^{2}\left(\rho^{n+1}, e^{a}\right)
\end{array}
$$


As a last step, our numerical method solves the following pressure correction equations:

$$
\begin{array}{r}
\boldsymbol{u}^{n+1}=\boldsymbol{u}^{a}-\Delta t \frac{\nabla P^{n+1}}{\rho^{n+1}} \\
E^{n+1}=E^{a}-\Delta t \frac{\nabla \cdot\left(\boldsymbol{u}^{n+1} P^{n+1}\right)}{\rho^{n+1}} \\
P^{n+1}=p\left(\rho^{n+1}, e^{a}\right)+\Delta t \rho^{n+1}\left(c^{2}\right)^{a} \nabla \cdot \boldsymbol{u}^{n+1}
\end{array}
$$

Equations (4.2) and (4.4) are decoupled by first taking the divergence of both sides of (4.2) and substituting the resulting expression for $\nabla \cdot \boldsymbol{u}^{n+1}$ into (4.4). We arrive at the following equation for $P^{n+1}$ :

$$
P^{n+1}-\rho^{n+1}\left(c^{2}\right)^{a} \Delta t^{2} \nabla \cdot\left(\frac{\nabla P^{n+1}}{\rho^{n+1}}\right)=p^{a}-\rho^{n+1}\left(c^{2}\right)^{a} \Delta t \nabla \cdot \boldsymbol{u}^{a}
$$

Once (4.5) is solved, the pressure correction is applied in (4.2-4.4).

The cell-centered advective velocity, $\boldsymbol{u}_{i}^{a}$, is an approximation to the cellintegrated average of velocity over the cell $\Omega_{i}$. In order to discretize $\nabla \cdot \boldsymbol{u}^{a}$, the face-centered advective velocity, $u_{i+1 / 2}^{a}$, is derived from $\boldsymbol{u}_{i}^{a}$ in such a way as to conserve momentum. The face centered advective velocity is the massweighted interpolation of the cell centered advective velocity (4.6):

$$
u_{i+1 / 2}^{a}=\frac{u_{i}^{a} \rho_{i, R}\left|\Omega_{i, R}\right|+u_{i+1}^{a} \rho_{i+1, L}\left|\Omega_{i+1, L}\right|}{\rho_{i+1 / 2}\left|\Omega_{i+1 / 2}\right|} .
$$

$\Omega_{i, R}$ is the right half control volume of cell $i$ and $\Omega_{i+1, L}$ is the left half control volume of cell $i+1$. The face centered control volume $\Omega_{i+1 / 2}(4.7)$ is,

$$
\Omega_{i+1 / 2}=\Omega_{i, R} \cup \Omega_{i+1, L}
$$

See Figure 4.

The density over each half control volume is:

$$
\begin{gathered}
\rho_{i, R}=\frac{1}{\left|\Omega_{i, R}\right|} \sum_{m=1}^{M}\left|\Omega_{i}^{m} \cap \Omega_{i, R}\right| \rho_{i}^{m, n+1} \\
\rho_{i+1, L}=\frac{1}{\left|\Omega_{i+1, L}\right|} \sum_{m=1}^{M}\left|\Omega_{i+1}^{m} \cap \Omega_{i+1, L}\right| \rho_{i+1}^{m, n+1}
\end{gathered}
$$




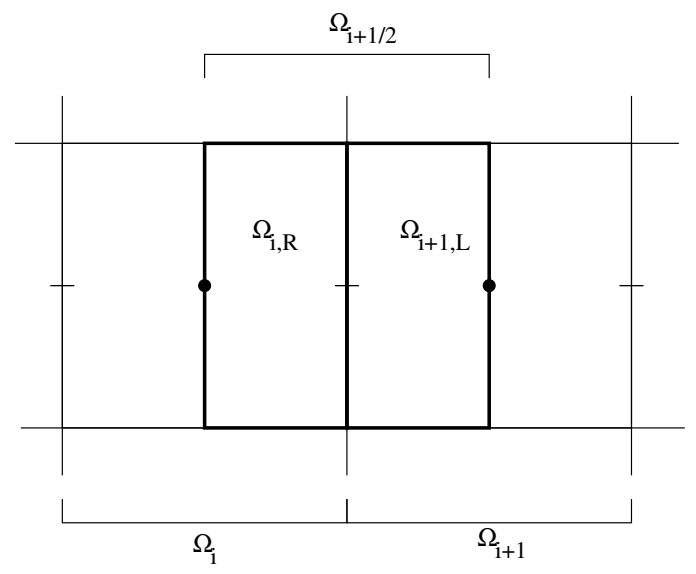

Figure 4: Left and right control volumes $\Omega_{i, R}, \Omega_{i+1, L}$, with face-centered control volume $\Omega_{i+1 / 2}$. Cell centers are shown as dots, and cell faces are shown as dashes.

The face centered density $\rho_{i+1 / 2}$ is defined as the mass in the half-cell regions $\Omega_{i, R}$ and $\Omega_{i+1, L}$ divided by the volume of the face-centered control volume $\Omega_{i+1 / 2}$, as in (4.10).

$$
\rho_{i+1 / 2}=\frac{\rho_{i, R}\left|\Omega_{i, R}\right|+\rho_{i+1, L}\left|\Omega_{i+1, L}\right|}{\left|\Omega_{i+1 / 2}\right|}
$$

See Figure 5.

A finite volume-style discretization of (4.5) is used. Divergence terms are integrated over the control volume $\Omega$ and divided by the magnitude of the control volume. For smooth solutions, this will converge to the divergence operator as the magnitude of the control volume goes to zero.

$$
\nabla \cdot\left(\frac{\nabla P}{\rho}\right) \approx \frac{\int_{\Omega} \nabla \cdot\left(\frac{\nabla P}{\rho}\right) d x}{\int_{\Omega} d x}=\frac{\int_{\partial \Omega}\left(\frac{\partial P}{\partial n} / \rho\right) d x}{\int_{\Omega} d x}
$$

The pressure gradient at the cell face is discretized using centered differencing. The $\nabla \cdot u^{a}$ term is handled similarly.

$$
\nabla \cdot u^{a} \approx \frac{\int_{\partial \Omega} u \cdot n d x}{\int_{\Omega} d x}
$$




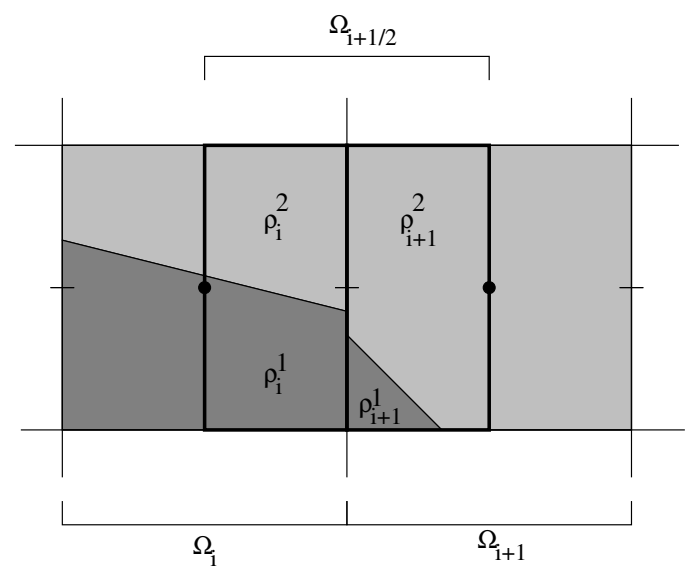

Figure 5: To compute the half cell density $\rho_{i, R}$ and $\rho_{i+1, L}$ in cut cells, the moment-of-fluid reconstructed interface is used to determine the half cell volume fractions $F_{i, R}^{m}$ and $F_{i+1, L}^{m}$, so that one can derive $\rho_{i, R}=\sum_{m} F_{i, R}^{m} \rho_{i}^{m}$ and $\rho_{i+1, L}=\sum_{m} F_{i+1, L}^{m} \rho_{i+1}^{m}$.

In $1 \mathrm{D}$, scheme (4.5) can then be written in fully discretized form (4.13):

$$
\begin{gathered}
P_{i}^{n+1}-\rho^{n+1}\left(c^{2}\right)_{i}^{a} \Delta t^{2}\left(\frac{\left(\frac{P_{i+1}^{n+1}-P_{i}^{n+1}}{\rho_{i+1 / 2}^{n+1} \Delta x}\right)-\left(\frac{P_{i}^{n+1}-P_{i-1}^{n+1}}{\rho_{i-1 / 2}^{n+1} \Delta x}\right)}{\Delta x}\right)= \\
p_{i}^{a}-\rho^{n+1}\left(c^{2}\right)_{i}^{a} \Delta t \frac{u_{i+1 / 2}^{a}-u_{i-1 / 2}^{a}}{\Delta x} \\
\rho^{n+1}\left(c^{2}\right)^{a}=\rho^{n+1} c^{2}\left(e_{i n t}^{m^{*}, a}, \rho^{m^{*}, n+1}\right)
\end{gathered}
$$

Once the linear system, (4.13), is inverted in order to determine a provisional pressure $P^{n+1}$, the pressure correction terms are computed in order to update the cell velocity $\boldsymbol{u}_{i, j}^{n+1}$ (4.2), face velocity $\left(u_{i+1 / 2, j}^{n+1}, v_{i, j+1 / 2}^{n+1}\right)(4.2)$ and energy $E_{i, j}^{n+1}$ (4.3). In 1D, (4.13) yields a tridiagonal system that is solved using the Thomas algorithm. In higher dimensions, a Multigrid-Preconditioned Conjugate Gradient method is used to solve the system [15]. 
The face velocity, $u_{i+1 / 2}^{n+1}$, is updated as follows:

$$
\frac{u_{i+1 / 2}^{n+1}-u_{i+1 / 2}^{a}}{\Delta t}=-\frac{P_{i+1}^{n+1}-P_{i}^{n+1}}{\rho_{i+1 / 2}^{n+1} \Delta x}
$$

The cell-averaged momentum and energy must be updated in a conservative fashion, so that correct shock speeds are calculated. It is required to interpolate the cell centered pressure to the cell faces. As in [36], we define the momentum equation in each half cell region, $\Omega_{i, R}$ and $\Omega_{i+1, L}$ :

$$
\frac{D u_{i, R}}{D t}=\frac{u_{i, R}^{n+1}-u_{i, R}^{a}}{\Delta t}=-\frac{P_{i+1 / 2}^{n+1}-P_{i}^{n+1}}{\rho_{i, R}^{n+1} \Delta x / 2}
$$

and

$$
\frac{D u_{i+1, L}}{D t}=\frac{u_{i+1, L}^{n+1}-u_{i+1, L}^{a}}{\Delta t}=-\frac{P_{i+1}^{n+1}-P_{i+1 / 2}^{n+1}}{\rho_{i+1, L}^{n+1} \Delta x / 2} .
$$

Applying the constraint that the interface between cells must remain in contact [36] implies $\frac{D u_{i, R}}{D t}=\frac{D u_{i+1, L}}{D t}$. Using this constraint and Eqn $(4.16-4.17)$, the pressure at the cell face $P_{i+1 / 2}$ can be found.

$$
P_{i+1 / 2}=\frac{\rho_{i, R} P_{i+1}+\rho_{i+1, L} P_{i}}{\rho_{i, R}+\rho_{i+1, L}}
$$

The derivations of the half cell densities, $\rho_{i, R}$ and $\rho_{i+1, L}$, are given by (4.8) and (4.9).

With pressure defined at cell faces, we can conservatively update the cell-averaged momentum and energy $(4.19,4.20)$ :

$$
\begin{aligned}
\frac{u_{i}^{n+1}-u_{i}^{a}}{\Delta t} & =-\frac{P_{i+1 / 2}^{n+1}-P_{i-1 / 2}^{n+1}}{\rho_{i}^{n+1} \Delta x} \\
\frac{E_{i}^{m, n+1}-E_{i}^{m, a}}{\Delta t} & =-\frac{(P u)_{i+1 / 2}^{n+1}-(P u)_{i-1 / 2}^{n+1}}{\rho_{i}^{n+1} \Delta x}
\end{aligned}
$$

Remarks:

- A one parameter family of consistent "asymptotically preserving" numerical methods for solving compressible multiphase flow problems can be defined by replacing the pressure correction equation (4.4) with the 
following generalized pressure correction formulation parameterized by $\beta \geq 1$ :

$$
P^{n+1}=p\left(\rho^{n+1}, e^{a}\right)+\beta \Delta t \rho^{n+1}\left(c^{2}\right)^{a} \nabla \cdot \boldsymbol{u}^{n+1} \quad \beta \geq 1
$$

In Figure 10 we report the sensitivity of results due to varying $\beta$ for the Sod shock tube benchmark problem; piecewise constant reconstruction of conserved variables is implemented. Increasing $\beta$ adds numerical dissipation. For future research, we would like to determine the values of $\beta$ that guarantee a monotonicity preserving[24] method. Figure 10 indicates that $\beta=1$ works the best, so that is the value that we use in all of the simulations reported in this paper.

- In the limit of infinite sound speed, i.e. $c^{2} \rightarrow \infty$, the pressure correction equations (4.2) and (4.4) reduce to the projection method for incompressible flows (see e.g. Chorin [13]).

- In contrast to the pressure equation that one must solve for incompressible flow, a solvability condition for (4.5) is unnecessary regardless of the boundary conditions for $P^{n+1}$.

- The modifications of our pressure correction algorithm in order to account for viscous and surface tension forces are as follows (validation of our algorithm for computing multiphase flows with surface tension and viscous forces are presented in sections 6.8 and 6.9):

1. Do the CISL advection step as outlined in (3.22-3.26).

2. Compute intermediate velocity $\boldsymbol{u}^{*}$ and intermediate energy $E^{m, *}$ $(m=1 \ldots M)$ :

$$
\begin{array}{r}
\frac{\boldsymbol{u}^{*}-\boldsymbol{u}^{a}}{\Delta t}=\frac{\nabla \cdot \tau\left(\boldsymbol{u}^{a}\right)}{\rho^{n+1}} \\
\frac{E^{m, *}-E^{m, a}}{\Delta t}=\frac{\nabla \cdot\left(\boldsymbol{u}^{a} \cdot \tau\left(\boldsymbol{u}^{a}\right)\right)}{\rho^{n+1}}
\end{array}
$$

The viscosity coefficient at cell faces is

$$
\mu_{i+1 / 2}=\frac{\left|\Omega_{i+1 / 2}\right|}{\sum_{m=1}^{M} \frac{1}{\mu_{m}}\left(\left|\Omega_{i}^{m} \cap \Omega_{i, R}\right|+\left|\Omega_{i+1}^{m} \cap \Omega_{i+1, L}\right|\right)}
$$


If the diffusion time step $\Delta t_{\text {diffuse }}$ associated with (4.22) is smaller than $\Delta t$, then a sub-cycling procedure is implemented for (4.22) and (4.23) in which the sub-cycling timestep is chosen to be smaller than $\Delta t_{\text {diffuse }}$.

3. Calculate the conservative pressure correction for momentum and energy the same as in (4.2-4.4) except for the following changes:

(a) replace $\boldsymbol{u}^{a}$ with $\boldsymbol{u}^{*}$ in (4.2).

(b) replace $E^{a}$ with $E^{*}$ in (4.3).

(c) construct a temporary signed distance function, $\phi . \phi_{i, j}$ is the signed distance from the cell $\boldsymbol{x}_{i, j}$ to the piecewise linear (planar in 3D) moment of fluid reconstructed interface:

$$
\phi_{i, j}=\left\{\begin{array}{cc}
+\left|\boldsymbol{x}_{i, j}-\boldsymbol{x}_{\text {closest }}\right| & \boldsymbol{x}_{i, j} \in \text { liquid } \\
-\left|\boldsymbol{x}_{i, j}-\boldsymbol{x}_{\text {closest }}\right| & \boldsymbol{x}_{i, j} \in \text { gas }
\end{array}\right.
$$

See Figure 6. The algorithm for finding the exact signed distance is explained in [50].

(d) The surface tension force is discretized similarly as in [29] in which the Ghost Fluid Method[34] is used. Below we describe our procedure in the $x$ direction, which has an analogous description in the $y$ or $z$ directions:

i. For the equation for $P_{i}^{n+1}$ in (4.13), the equation for $P_{i \pm 1 / 2}$ in (4.18) when updating $u_{i}^{n+1}$ in (4.19), the equation for $P_{i \pm 1 / 2}$ in (4.18) when updating $E_{i}^{m, n+1}$ in (4.20), and when updating $u_{i+1 / 2}^{n+1}$ in (4.15), we replace $P_{i \pm 1}^{n+1}$ with,

$$
P_{i \pm 1}^{n+1}+\sigma \kappa_{i \pm 1 / 2} \frac{H\left(\phi_{i \pm 1}\right)-H\left(\phi_{i}\right)}{\Delta x}
$$

where $\sigma$ is the surface tension coefficient, $\kappa_{i \pm 1 / 2}$ is the interfacial curvature (4.27) discretized using the height function technique, and $H(\phi)$ is the Heaviside function,

$$
H(\phi)= \begin{cases}1 & \phi \geq 0 \\ 0 & \phi<0\end{cases}
$$

ii. The level set height function method [5, 49] is used to approximate the curvature. Referring to Figure $6, \kappa_{i \pm 1 / 2}$ 
in (4.25) is approximated as follows:

$$
\begin{array}{r}
\kappa_{i \pm 1 / 2}=\left\{\begin{array}{cc}
\kappa_{i} & \left|\phi_{i}\right|<\left|\phi_{i \pm 1}\right| \\
\kappa_{i \pm 1} & \text { otherwise }
\end{array}\right. \\
h^{\prime \prime} \approx \frac{h_{i+1}-2 h_{i}+h_{i-1}}{\Delta x^{2}} \quad h^{\prime} \approx \frac{h_{i+1}-h_{i-1}}{2 \Delta x} \\
\kappa_{i}=\frac{-h^{\prime \prime}}{\left(1+\left(h^{\prime}\right)^{2}\right)^{3 / 2}}
\end{array}
$$
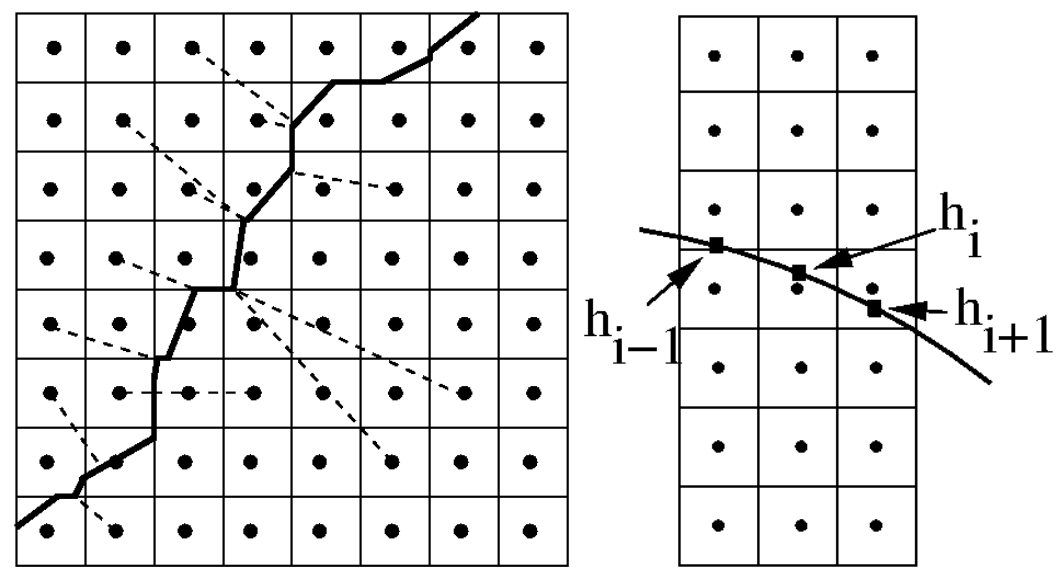

Figure 6: Left: A temporary level set function $\phi$ is the exact signed distance to the piecewise linear Moment-of-Fluid reconstructed interface. Right: The level set height function method is used to approximate the curvature of the interface. The square symbols are located at the zero crossings of $\phi$; e.g. $h_{i}=\left(1-\theta_{i, j+1 / 2}\right) y_{j}+\theta_{i, j+1 / 2} y_{j+1}$ where $\theta_{i, j+1 / 2} \equiv \frac{\left|\phi_{i, j}\right|}{\left|\phi_{i, j}\right|+\left|\phi_{i, j+1}\right|}$.

\section{$5 \quad$ Fixed-Point Face Velocity Iterations}

For a 1D single material problem, suppose the reconstructed slope is taken to be zero in all cells, face velocity in a given cell is constant $\left(u_{i \pm 1 / 2}=c\right)$, and the CFL number $C F L=c \frac{\Delta t}{\Delta x}<1 / 2$. Then, it can be shown that the Cell-Integrated Semi-Lagrangian method is equivalent to the dissipative first order Forward Euler method. However, if the face velocity is not divergence free, and we use the face velocity computed from the previous time step, $\boldsymbol{u}_{\text {face }}^{n}$, in order to define the backward-traced characteristics (i.e. in order 
to define the departure volumes), then we observe instability manifested by ringing at shocks. We have also observed instability for flows that are close to incompressible.

We have found it critical to iterate at least once through a complete advection and pressure correction cycle in order to predict the advection velocity at time $t=t^{n+1}$. We remark that in the work of Gretarsson and Fedkiw[21], they have implemented high order Runge-Kutta schemes which we think achieves the same goal of defining an accurate time advanced prediction to the advection velocity.

We have implemented a fixed point procedure in order to define the backward-traced characteristics. Let superscript $k$ denote the state variable at the $k^{\text {th }}$ fixed-point iteration. Iteration $u_{\text {face }}^{n+1,(0)}$ is defined as $u_{\text {face }}^{n}$.

1. $\boldsymbol{U}^{n+1,(k+1)}=\operatorname{ADVECT}\left(\boldsymbol{U}^{n}, \boldsymbol{u}_{\text {face }}^{n+1,(k)}\right)$

2. $\boldsymbol{u}^{a,(k)}=\frac{(\rho \boldsymbol{u})^{n+1,(k+1)}}{(\rho)^{n+1,(k+1)}}, E^{a,(k)}=\frac{(\rho E)^{n+1,(k+1)}}{(\rho)^{n+1,(k+1)}}$.

3. $p^{a,(k)}=\operatorname{EOS}\left(\rho^{n+1,(k+1)}, e_{\text {int }}^{a,(k)}\right)$

4. $\boldsymbol{u}_{\text {face }}^{n+1,(k+1)}, \boldsymbol{u}^{n+1,(k+1)}, E^{n+1,(k+1)}, P^{n+1,(k+1)}=$

$\operatorname{PRESSURE~CORRECTION~}\left(p^{a,(k)}, \rho^{n+1,(k+1)}, \boldsymbol{u}^{a,(k)}\right)$

5. Set $k=k+1$.

Our numerical tests indicate that while the diffusion associated with the fixed-point procedure causes a slight loss of sharpness at the shock front, the resulting solution exhibits much lower total variation, which is a physically desirable characteristic [37]. Convergence in the error of the fixed-point iteration is observed for shock tube problems after only two iterations. This fixed-point method is applied in multiple dimensions too, always using just two sweeps. Results for the Spherical Explosion Shock (Sec. 6.5) exhibited much less oscillatory behavior when using multiple sweeps. Results for the Spherical Explosion Shock and Oscillating Water Column demonstrate correct shock/interface speeds in both high and low Mach number problems. We demonstrate shock resolution using our first order temporal discretization that is comparable in accuracy to methods that use high order shock capturing techniques. 
The reduction in numerical oscillations by using $\boldsymbol{u}^{n+1}$ instead of $\boldsymbol{u}^{n}$ in (5.1) can be explained by looking at the leading order terms in the error for pressure for the following semi-discrete method:

$$
\begin{array}{r}
\frac{D \rho}{D t}=-\rho \nabla \cdot \boldsymbol{u}^{n+1} \\
\frac{D \boldsymbol{u}}{D t}=-\frac{\nabla p^{n+1}}{\rho} \\
\frac{D e}{D t}=-\frac{p}{\rho} \nabla \cdot \boldsymbol{u}^{n+1} \\
\frac{D p}{D t}=p_{\rho} \frac{D \rho}{D t}+p_{e} \frac{D e}{D t}=-\rho c^{2} \nabla \cdot \boldsymbol{u}^{n+1}= \\
-\rho c^{2} \nabla \cdot\left(\boldsymbol{u}^{a}-\Delta t \frac{\nabla p^{n+1}}{\rho}\right)= \\
-\rho c^{2} \nabla \cdot \boldsymbol{u}^{a}+\Delta t \rho c^{2} \nabla \cdot \frac{\nabla p^{n+1}}{\rho} .
\end{array}
$$

The term in (5.2),

$$
\Delta t \rho c^{2} \nabla \cdot \frac{\nabla p^{n+1}}{\rho}
$$

is a numerical diffusive term for the pressure equation,

$$
\frac{D p}{D t}=-\rho c^{2} \nabla \cdot \boldsymbol{u}+\Delta t \rho c^{2} \nabla \cdot \frac{\nabla p^{n+1}}{\rho} .
$$

\section{$6 \quad$ Numerical Results}

The acoustic time step $\Delta t_{u+c}$ is chosen according to the following scheme.

$$
\Delta t_{u+c}^{n+1}= \begin{cases}\Delta t^{*}=C F L \frac{\Delta x}{\max _{i}\left(\left|u_{i}\right|+c_{i}\right)}, & \Delta t^{*} \leq \operatorname{cmax} \cdot \Delta t^{n} \\ \operatorname{cmax} \cdot \Delta t^{n}, & \text { otherwise }\end{cases}
$$

The advective time step $\Delta t_{u}$ does not consider the sound speed:

$$
\Delta t_{u}^{n+1}= \begin{cases}\Delta t^{*}=C F L \frac{\Delta x}{\max _{i}\left(\left|u_{i}\right|\right)}, & \Delta t^{*} \leq \operatorname{cmax} \cdot \Delta t^{n} \\ \operatorname{cmax} \cdot \Delta t^{n}, & \text { otherwise }\end{cases}
$$


The factor cmax denotes the maximum allowed increase in the time step from one step to the next. For all tests presented, we set cmax equal to 1.025 .

The initial time step at $t=0, \Delta t^{0}$, is defined as follows:

- We calculate $\Delta t^{0}$ either using the advection time step,

$$
\Delta t_{u}^{0}=C F L \frac{\Delta x}{\max _{i}\left(\left|u_{i}\right|\right)},
$$

or the acoustic time step,

$$
\Delta t_{u+c}^{0}=C F L \frac{\Delta x}{\max _{i}\left(\left|u_{i}\right|+c_{i}\right)} .
$$

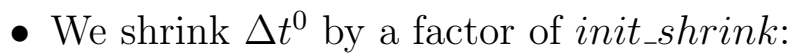

$$
\Delta t^{0} \leftarrow \text { init_shrink } \Delta t^{0}
$$

The factor init_shrink accounts for the fact that we do not know $a$ priori what the time step should be, especially in the case when an initial pressure gradient is prescribed and the initial velocity is zero. The value init_shrink $=0.1$ is used in all of our simulations.

For single dimensional problems, a uniform grid is used, but for multidimensional test problems, we use dynamic block structured adaptive mesh refinement in order to define the computational grid [48, 47] (AMR). A computational domain that is organized using AMR is made up of a hierarchy of adaptive levels $\ell=0, \ldots, \ell^{\max }$ with each level being the union of disjoint rectangular grids. Level $\ell=0$ is the coarsest level and the mesh size on each finer level is half the mesh size of the preceding level, $\Delta x^{\ell+1}=\Delta x^{\ell} / 2$.

Referring to figure (7), filled circles represent cells or faces not hidden by a finer mesh. Open circles are either hidden coarse level cells or faces or fictitious fine grid cells. The solution at cells or faces corresponding to open circles are interpolated from the solution at cells or faces corresponding to filled circles. Coarse and fine levels are synchronized by "averaging down" the fine level solution onto the coarser level. For example, the hidden coarse grid cell value at cell " 3 " is the volume weighted average of the finer level cells "5," "6," "7," and "8." It could be that the stencil for the fine level cell "6" 
includes the fictitious fine level cell "9." In this case, the fictitious cell "9" value is interpolated from the coarse level values at cells " 1, " "2," "3," and "4." The algorithm used for interpolating volume fraction and moment data from coarse levels to fine levels, and the algorithm used for averaging down volume fraction and moment data from fine level cells to hidden coarse level cells is identical to that reported in [29]. Density, momentum, and energy are interpolated from coarse to fine levels using piecewise constant interpolation. In order to maintain conservation at coarse/fine boundaries when implementing our CISL advection algorithm, we solve (3.22-3.26) in fictitious fine grid cells (dashed cells in Figure 7) and then replace the underlying coarse grid solution (cells "1" and "2") with the appropriate mass/volume weighted average of the fictitious fine grid solution.

The discretization of the pressure correction equation (4.13) requires the initialization of fine grid ghost cell values (e.g. cell "9"). This is achieved by bilinear interpolation of points "1," "2," "3," "4." Cells "3" and "4," since they are hidden by the finer level, are first initialized as the volume weighted average of the pressure from the appropriate fine level cells. $\nabla p / \rho$ at face "12" is defined as the area weighted average of $\nabla p / \rho$ at faces "10" and "11."

Let $L_{\text {bilinear }} p$ represent the linear operator on the AMR grid in which bilinear interpolation is used to define ghost pressure values at a coarse/fine grid boundary. $L_{\text {bilinear }}$ is not a symmetric operator so that we cannot directly use the multigrid preconditioned conjugate gradient for inverting

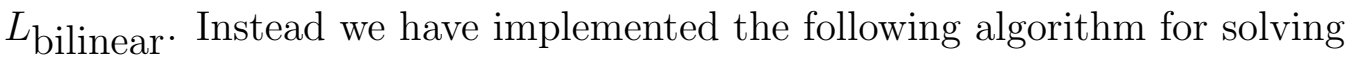

$$
L_{\text {bilinear }} p=b .
$$

Given $p^{(0)}, r^{(0)}=b-L_{\text {bilinear }} p^{(0)}, k=0$

1. solve $L_{\text {piecewise_constant }} \delta p=r^{(k)}$ using the AMR multigrid preconditioned conjugate gradient method. The matrix operator associated with piecewise constant interpolation at coarse/fine grid boundaries, $L_{\text {piecewise_constant, }}$ corresponds to a symmetric matrix so that the Preconditioned Conjugate Gradient method is guaranteed to converge.

2. $p^{(k+1)}=p^{(k)}+\delta p, r^{(k+1)}=b-L_{\text {bilinear }} p^{(k+1)}$

3. $k=k+1$, if not converged, go back to step 1 . 


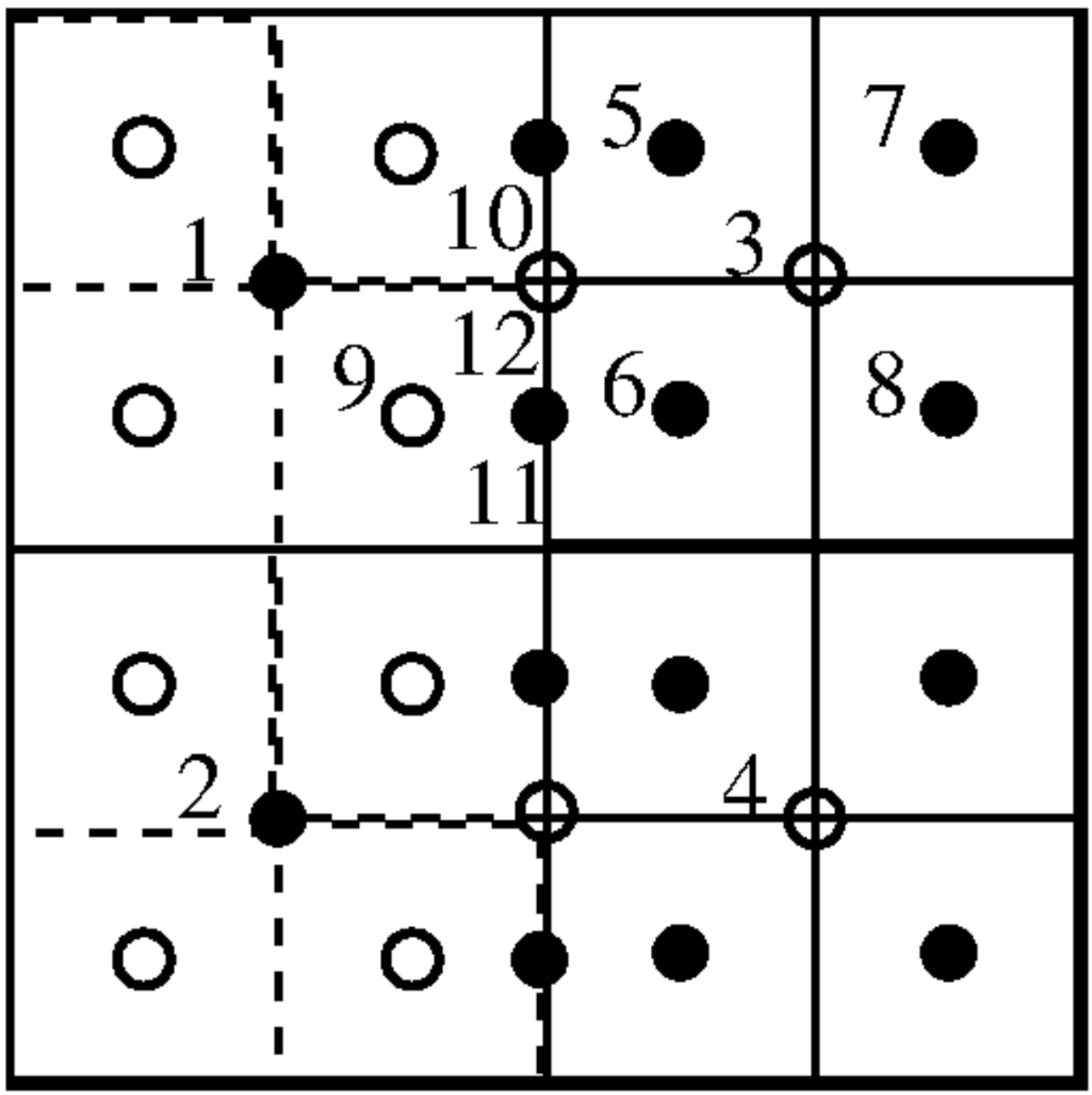

Figure 7: Illustration of a coarse/fine interface on an AMR grid. Filled circles represent cells or faces not hidden by a finer mesh. Open circles are either hidden coarse level cells or faces or fictitious fine grid cells.

\subsection{One Dimensional, Single Phase, Shock Tube and Shock-Turbulence Interaction Test Problems}

We test our new algorithm on the Sod and Strong Shock Tube test problems and on a shock/turbulence interaction test problem (see section 4.6 of [28]). In each case, the gas is governed by the ideal gas equation (6.6) with $\gamma=1.4$ 
and we prescribe zero viscosity.

$$
p=(\gamma-1) \rho e_{i n t}
$$

For the Sod and Strong Shock Tube problem, and the shock-turbulence interaction problem, the time step was determined from the acoustic time step (6.1) with $\mathrm{CFL}=1 / 2$. In other words the time step

$$
\Delta t=\frac{1}{2} \frac{\Delta x}{\max _{i}\left(\left|u_{i}\right|+c_{i}\right)}
$$

was used. For the Sod shock tube problem (6.7), we also report results when the acoustic time step with $\mathrm{CFL}=1.5$ is used.

The initial conditions for these three shock wave test problems are:

\section{Sod Shock tube}

$$
(\rho(x, 0), u(x, 0), p(x, 0))= \begin{cases}(1,0,1), & x \leq 0.5 \\ (0.125,0,0.1), & x>0.5\end{cases}
$$

Strong Shock tube

$$
(\rho(x, 0), u(x, 0), p(x, 0))= \begin{cases}\left(1,0,10^{10}\right), & x \leq 0.5 \\ (0.125,0,0.1), & x>0.5\end{cases}
$$

\section{Shock-Turbulence interaction}

$$
(\rho(x, 0), u(x, 0), p(x, 0))= \begin{cases}(3.857148,2.629369,10.333333), & 0 \leq x \leq 1 \\ (1+0.2 \sin (5 x-5), 0,1), & 1<x<10\end{cases}
$$

For the shock tube test cases, the error $E_{N}$ and rate of convergence $p_{N}$ are reported for density in Table 3; we use the analytical solution of the gas dynamics equations for a perfect gas as the reference solution to the Riemann problem. The error $E_{N}$ is the $L^{1}$ error and is defined as,

$$
\begin{array}{r}
E_{N}=\sum_{i=0}^{N-1}\left|\rho_{i}-\rho^{\operatorname{exact}}\left(x_{i}\right)\right| \Delta x . \\
x_{i}=(i+1 / 2) \Delta x
\end{array}
$$


The rate of convergence is defined as

$$
p_{N}=\log _{2}\left|\frac{E_{N / 2}}{E_{N}}\right|
$$

\begin{tabular}{|c|c|c|c|c|c|c|}
\hline $\mathrm{N}$ & $\begin{array}{c}\text { Sod } E_{N} \\
\text { CFL=0.5 }\end{array}$ & $\begin{array}{c}\text { Sod } p_{N} \\
\text { CFL=0.5 }\end{array}$ & $\begin{array}{c}\text { Sod } E_{N} \\
\text { CFL=1.5 }\end{array}$ & $\begin{array}{c}\text { Sod } p_{N} \\
\text { CFL=1.5 }\end{array}$ & $\begin{array}{c}\text { Strong } E_{N} \\
\text { CFL=0.5 }\end{array}$ & $\begin{array}{c}\text { Strong } p_{N} \\
\text { CFL=0.5 }\end{array}$ \\
\hline 50 & $1.875 \mathrm{e}-02$ & - & $2.126 \mathrm{e}-02$ & - & - & - \\
\hline 100 & $1.174 \mathrm{e}-02$ & 0.676 & $1.417 \mathrm{e}-02$ & 0.586 & $2.308 \mathrm{e}-02$ & - \\
\hline 200 & $7.291 \mathrm{e}-03$ & 0.687 & $1.031 \mathrm{e}-02$ & 0.458 & $1.326 \mathrm{e}-02$ & 0.800 \\
\hline 400 & $4.406 \mathrm{e}-03$ & 0.726 & $6.938 \mathrm{e}-03$ & 0.572 & $7.889 \mathrm{e}-03$ & 0.749 \\
\hline 800 & $2.634 \mathrm{e}-03$ & 0.743 & $4.415 \mathrm{e}-03$ & 0.652 & $4.607 \mathrm{e}-03$ & 0.776 \\
\hline 1600 & $1.592 \mathrm{e}-03$ & 0.726 & $2.785 \mathrm{e}-03$ & 0.665 & $2.761 \mathrm{e}-03$ & 0.739 \\
\hline
\end{tabular}

Table 3: $L^{1}$ error $E_{N}$ and convergence rate $p_{N}$ for density for the Sod Shock Tube problem at $t=0.15$ (6.7) and the Strong Shock Tube problem at $t=0.12$ (6.8). A linear MINMOD reconstruction for all conserved variables is used.

In Figures 8 through 14, we plot the solutions for the two shock tube problems (Sod $t=0.15$, Strong $t=2.5 E-6$ ) and for the shock-turbulence interaction problem $(t=1.8)$. We capture the correct shock speed and shock strength under grid refinement for these test problems. The errors reported in Table 3 show that the rate of convergence is about $p=3 / 4$ which is expected for a shock-capturing method.

Remarks:

- non-conservative schemes fail for this problem, due to their inability to correctly compute shock speeds [58].

- While the density jump for the Strong Shock tube problem at the material interface (see Figure 11) is not captured as sharply as in [21], less oscillation is observed at the head of the rarefaction wave.

- For the Sod shock tube problem, we illustrate the sensitivity of results due to different values for CFL; see Figure 9. Discontinuities are smeared as $\Delta t$ is increased beyond the acoustic time step constraint. 
- Also for the Sod shock tube problem, we illustrate the sensitivity of results due to different values for $\beta$ (4.21); see Figure 10. Discontinuities are smeared as $\beta$ is increased beyond $\beta=1$.

- For the shock-turbulence interaction problem, our method does not capture the shock ringing that appears downstream of the shock as well as the method developed in [28]. Although our method converges under grid refinement, the grid required to approximate the waves immediately downstream of the shock with a given accuracy is about twice as fine as in [28]. While our method performs worse in the smooth oscillatory regions, our method does capture the shock wave with a comparable number of grid points when compared to [28]. In our Figure 14 we show 3-4 cells spanning the shock transition region. In Figures 15-18 of [28], there are 2 cells spanning the shock transition region. The algorithm of [28] stores both the volume integrated average (VIA) and point value $(\mathrm{PV})$ for the conserved variables, which means there are about 4 degrees of freedom, same as with our algorithm (irrespective of grid resolution), spanning the shock transition region. 

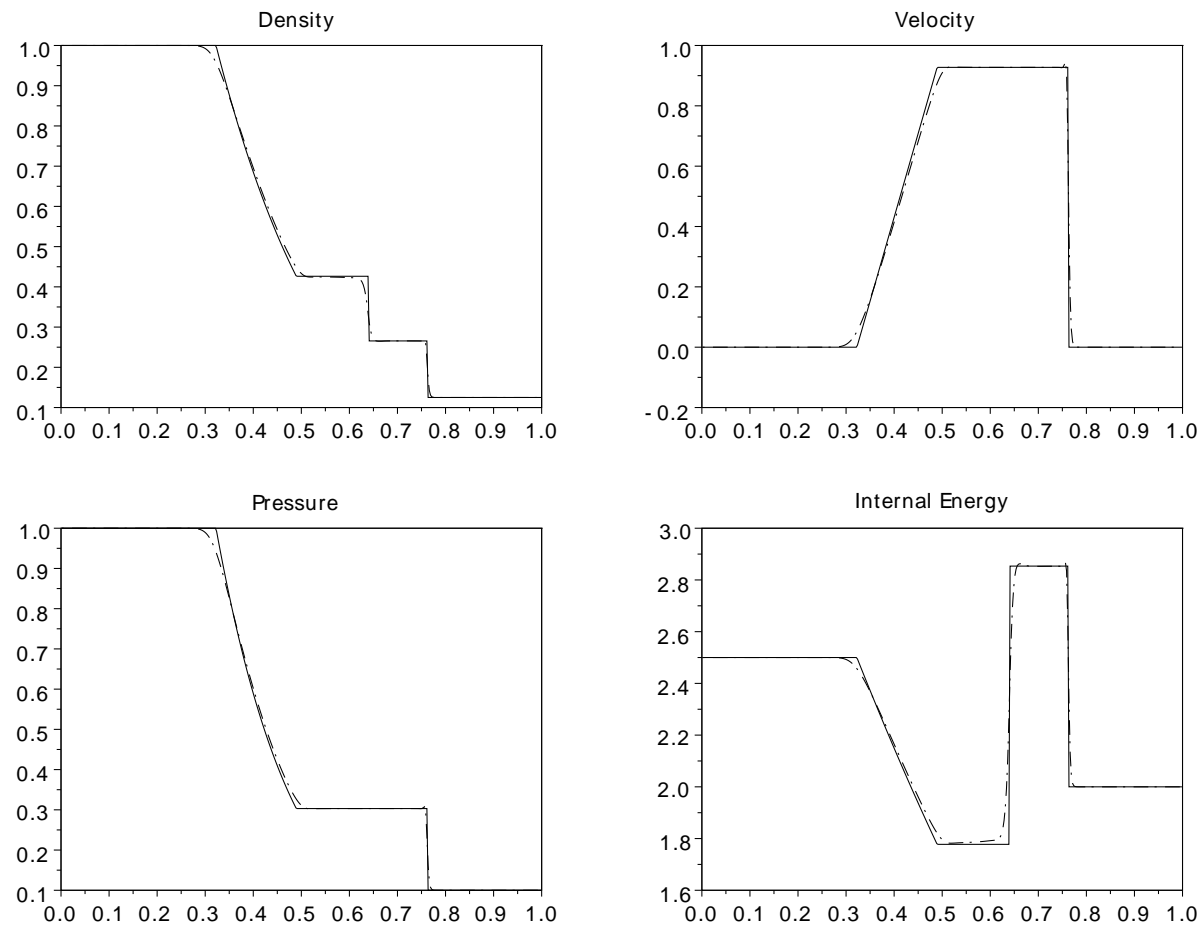

Figure 8: Sod Shock Tube at time $\mathrm{t}=0.15$. The solid line indicates the analytical solution to the Riemann problem. The dotted line is the reference solution for $\mathrm{N}=400$ cells. A linear MINMOD slope reconstruction for conserved variables is used. 


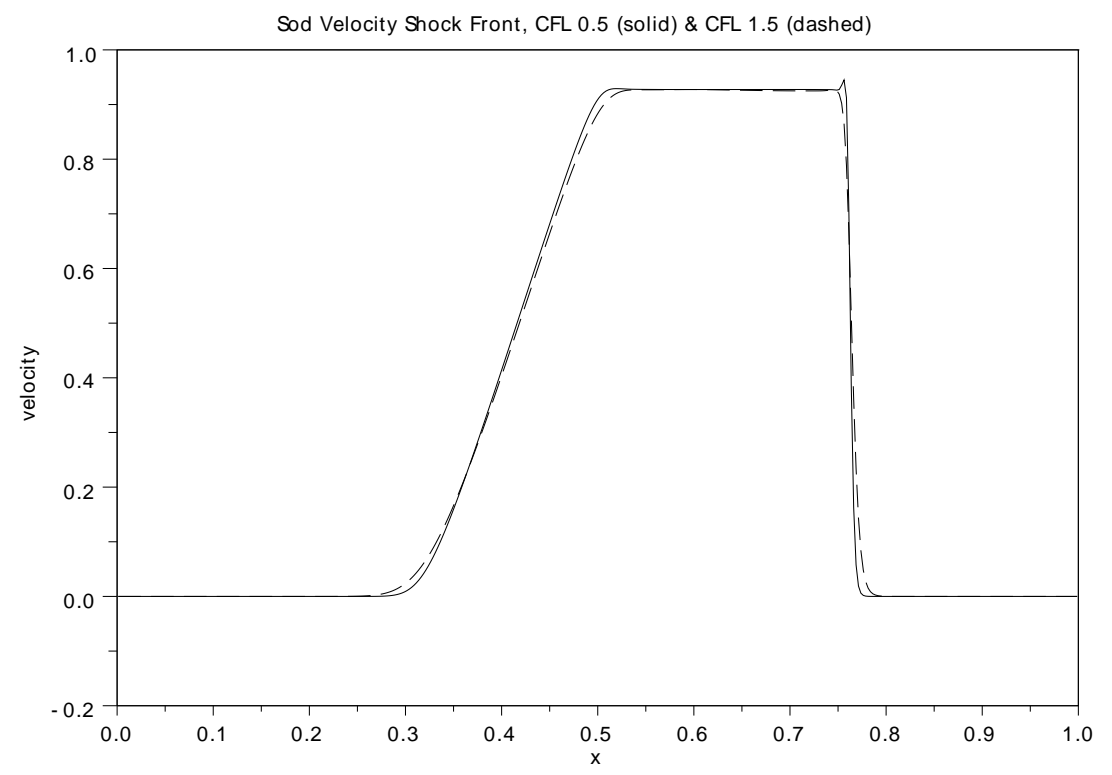

Figure 9: Velocity for the Sod Shock Tube at time $t=0.15$. The solid line indicates the numerical results using the acoustic time step constraint with $\mathrm{CFL}=0.5$ and the dashed line represent the results using $\mathrm{CFL}=1.5$. The number computational cells is $N=400$. A linear MINMOD slope reconstruction for conserved variables is used. 


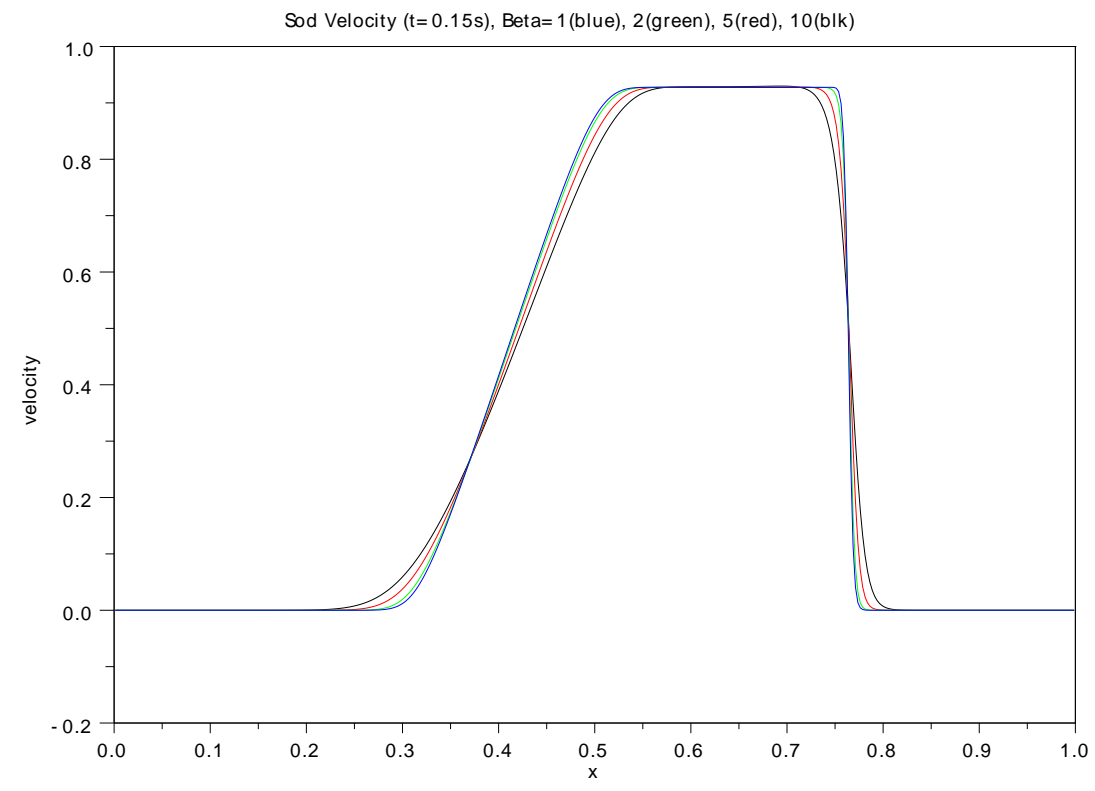

Figure 10: Effect of $\beta$ (4.21) on the simulation results for the Sod shock tube test problem at time $t=0.15$. Piecewise constant reconstruction is used for all conserved variables. The acoustic time step constraint with $\mathrm{CFL}=0.5$ is used. $N=400$. The blue curve corresponds to $\beta=1$, green $\beta=2$, red $\beta=5$, and black $\beta=10$. 

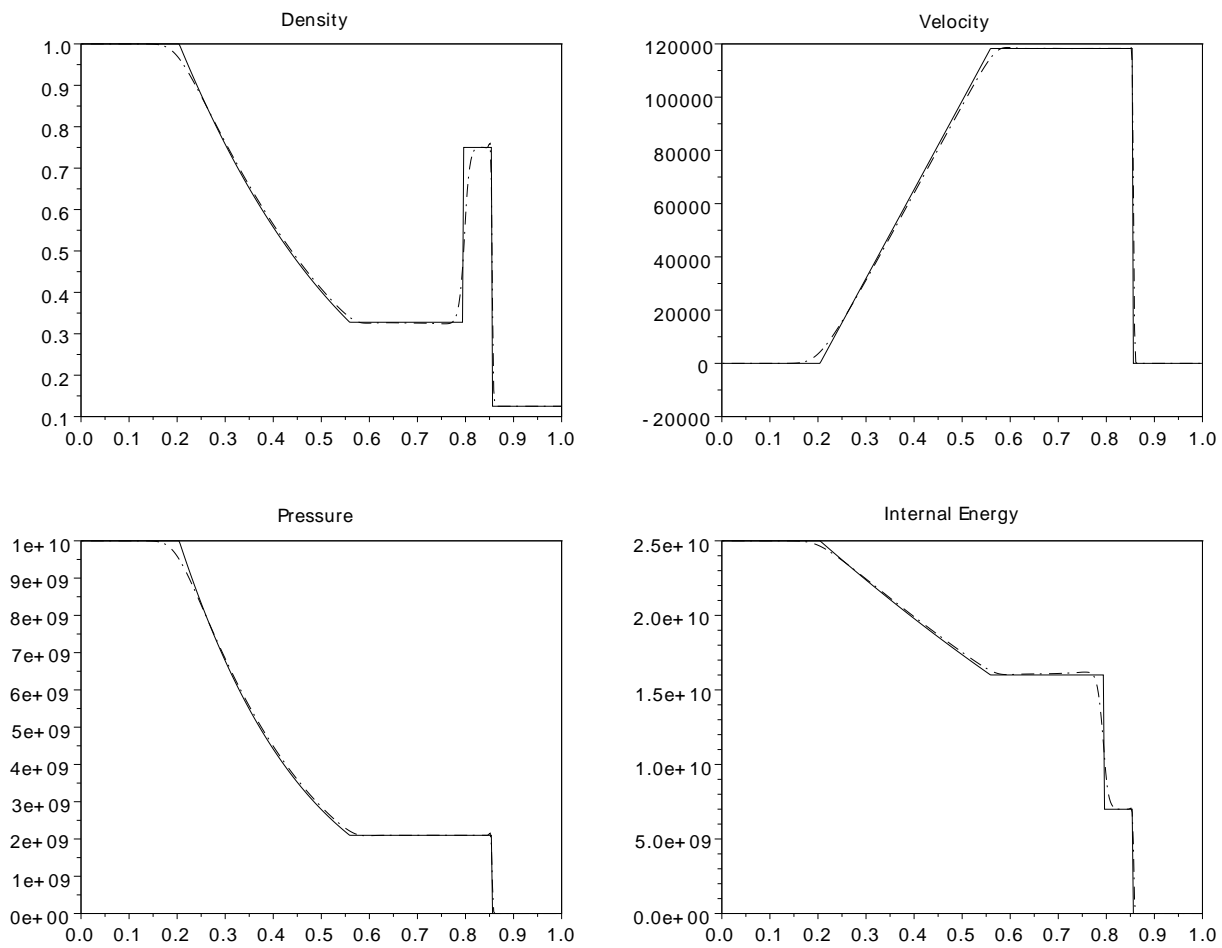

Figure 11: Strong Shock Tube at time $t=2.5 \mathrm{E}-6$. The solid line indicates the analytical solution to the Riemann problem. The dotted line is the reference solution for $\mathrm{N}=400$ cells. A linear MINMOD slope reconstruction for all conserved variables is used. 


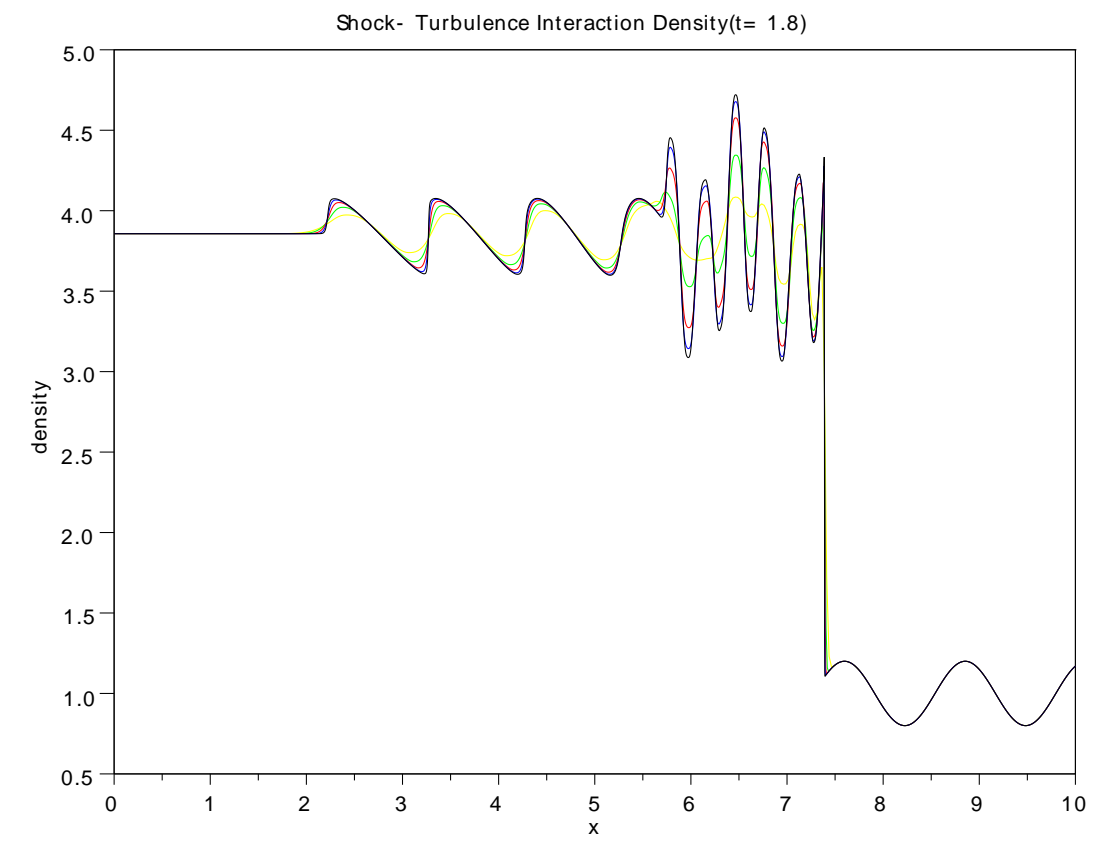

Figure 12: Shock-Turbulence interaction at time $\mathrm{t}=1.8$. Results for five different grid resolutions are compared: $N=400$ (yellow), $N=800$ (green), $N=1600$ (red), $N=3200$ (blue), and $N=6400$ (black). A linear MINMOD slope reconstruction for all conserved variables is used. 


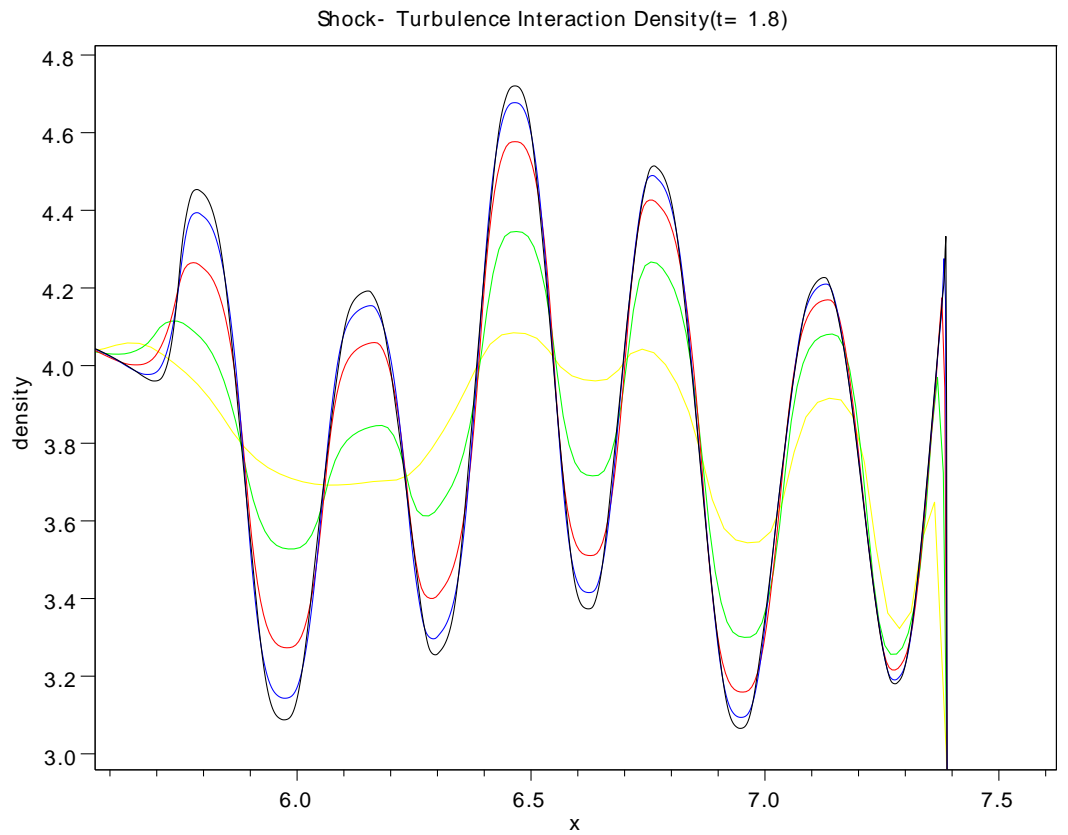

Figure 13: Shock-Turbulence interaction at time $t=1.8$. Zoomed in view just downstream of the shock wave. Results for five different grid resolutions are compared: $N=400$ (yellow), $N=800$ (green), $N=1600$ (red), $N=3200$ (blue), and $N=6400$ (black). A linear MINMOD slope reconstruction for all conserved variables is used. 


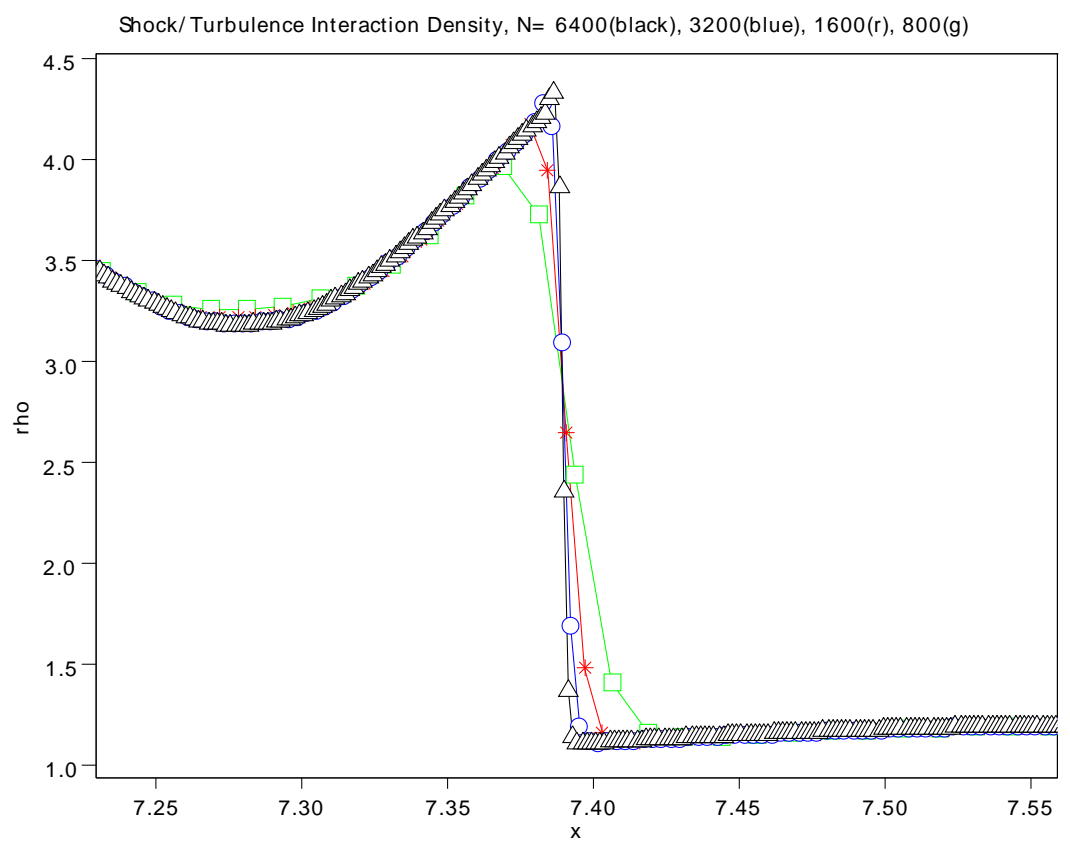

Figure 14: Shock-Turbulence interaction at time $\mathrm{t}=1.8$. Zoomed in view at the shock wave. Results for four different grid resolutions are compared: $N=800$ (green), $N=1600$ (red), $N=3200$ (blue), and $N=6400$ (black). A linear MINMOD slope reconstruction for all conserved variables is used. 


\subsection{Face Velocity Fixed Point Iterations}

The error and total variation (6.13) associated with using multiple advection+ pressure correction sweeps in order to predict an accurate, time advanced, face velocity is displayed in Table 4. Results are shown for 400 cells, with error taken in density.

$$
T V=\sum_{i=1}^{N}\left|\rho_{i}-\rho_{i-1}\right|
$$

Results for L1 error and Total Variation for the Sod and Strong Shock tube tests, shown in Table 4, indicate that the fixed point iteration has converged after 4 iterations. However, the relative difference in L1 error between using 5 fixed point iterations and 2 fixed point iterations is less than $3 \%$. Because the cost of performing additional fixed point iterations is large (for each sweep, all materials are transported and the pressure equation must be solved), and the change in the overall solution is small, only two fixed point iterations are used for all other results presented in this work. Figures $15-16$ show the convergence of the solution under the fixed point iteration, with results for 2 and 3 iterations are visually indistinguishable.

\begin{tabular}{|c|c|c|c|c|}
\hline & \multicolumn{2}{|c|}{ Sod } & \multicolumn{2}{c|}{ Strong } \\
\hline Sweeps & L1 Error & TV & L1 Error & TV \\
\hline 1 & $3.874 \mathrm{e}-03$ & 0.914 & $6.917 \mathrm{e}-03$ & 1.807 \\
\hline 2 & $4.406 \mathrm{e}-03$ & 0.882 & $7.889 \mathrm{e}-03$ & 1.750 \\
\hline 3 & $4.406 \mathrm{e}-03$ & 0.882 & $7.921 \mathrm{e}-03$ & 1.748 \\
\hline 4 & $4.407 \mathrm{e}-03$ & 0.882 & $7.924 \mathrm{e}-03$ & 1.748 \\
\hline 5 & $4.407 \mathrm{e}-03$ & 0.882 & $7.924 \mathrm{e}-03$ & 1.748 \\
\hline
\end{tabular}

Table 4: Errors and Total Variation for Sod and Strong Shock Tube problems, taken at final times $\mathrm{t}=0.15,2.5 \mathrm{E}-6$ respectively. 

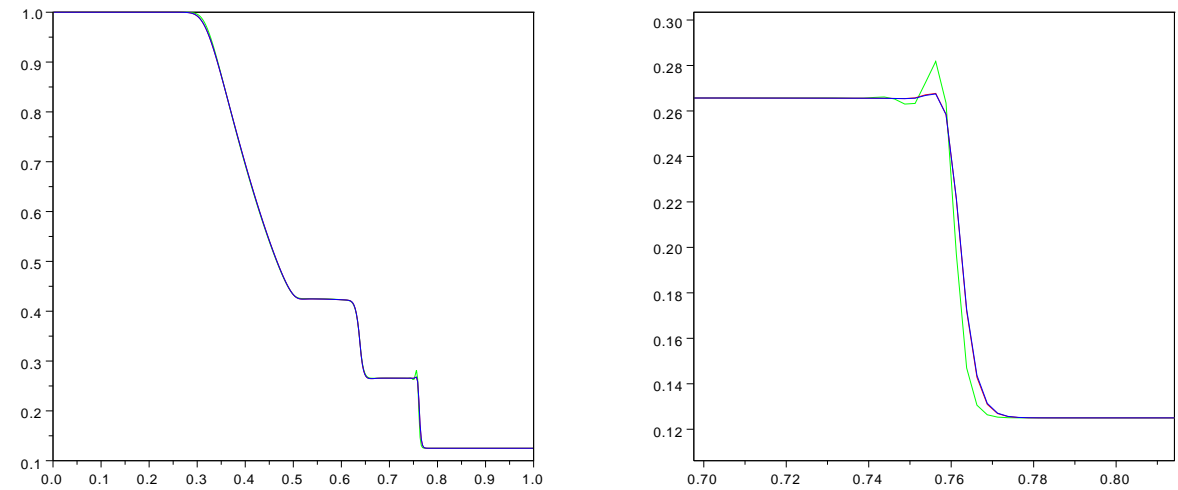

Figure 15: Sod Shock Tube at time $t=0.15$ using various fixed-point iteration sweeps. Green denotes one sweep, red denotes two sweeps, and blue denotes three sweeps. The right figure is zoomed to show overshoot at the shock front. A linear MINMOD slope reconstruction for all conserved variables is used.
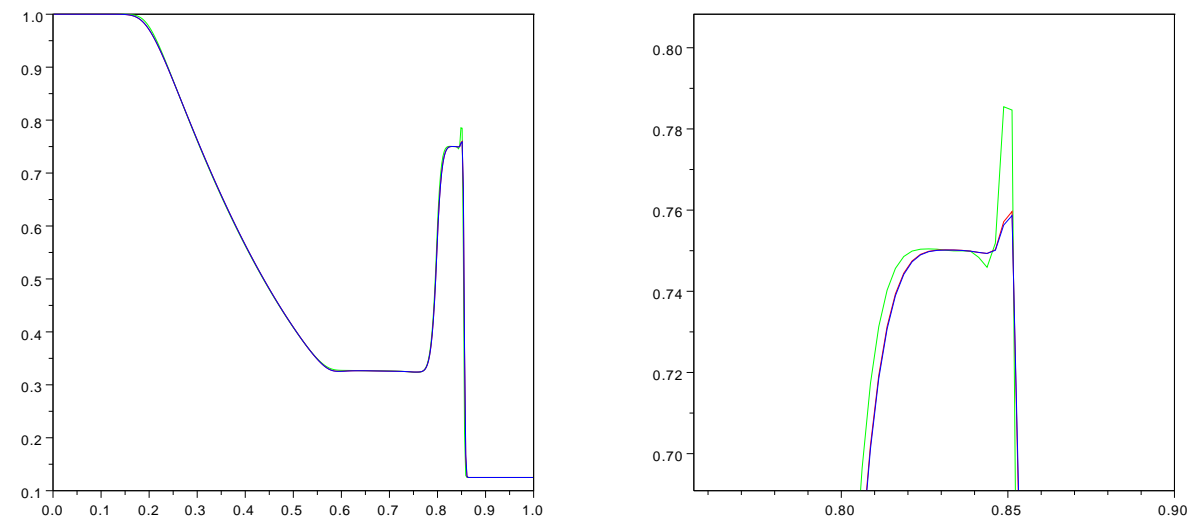

Figure 16: Strong Shock Tube at time $t=2.5 \mathrm{E}-6$ using various fixed-point iteration sweeps. Green denotes one sweep, red denotes two sweeps, and blue denotes three sweeps. The right figure is zoomed to show overshoot at the shock front. A linear MINMOD slope reconstruction for all conserved variables is used. 


\subsection{Oscillating Water Column}

A one-dimensional tube with reflecting boundaries at the left and right ends is considered. A column of inviscid water occupies the middle of the tube, with inviscid air on either end (Fig. 17). At time $t=0$, water begins moving from left to right with velocity one, compressing the gas to the right and expanding the gas to the left. This causes a difference in pressure across the system, which leads the material to decelerate, stop, and then accelerate moving from right to left. The equation of state (6.14) is used to govern both materials, with the appropriate coefficients taken for air and water.

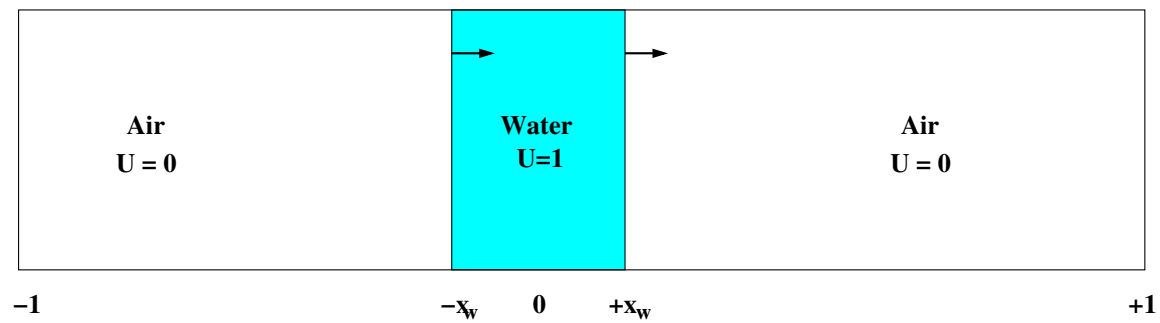

Figure 17: Initial configuration of the oscillating water column problem. Movement of the water compresses the air, causing flow to slow down and reverse direction.

$$
\frac{p+B p_{\text {ref }}}{(1+B) p_{\text {ref }}}=\left(\frac{\rho}{\rho_{\text {ref }}}\right)^{\gamma}
$$

As in Koren, et. al. [35] and Kadioglu, et. al. [32], the coefficients are taken as $B_{w}=3000, B_{a}=0, \rho_{\text {ref }, w}=1, \rho_{\text {ref }, a}=0.001, \gamma_{w}=7, \gamma_{a}=1.4, p_{\text {ref }, w}=$ $p_{r e f, a}=1$, and $x_{w}=0.1$ where the subscripts "w" and "a" correspond to water and air respectively. Initially, $p=1 \mathrm{in}$ both the air and the water. To be consistent with Kadioglu et al, the acoustic time step (6.1) with $C F L=3$ is used. At the very first time step, the velocity in the gas cells directly adjacent to the interface is initialized to one as well. A non-conservative momentum update is applied at cells adjacent to the walls. The update

$$
\begin{gathered}
u_{0}^{n+1}=\frac{\rho_{0, R}\left|\Omega_{0, R}\right| u_{0}^{a}}{\rho_{0, R}\left|\Omega_{0, R}\right|+\rho_{0, L}\left|\Omega_{0, L}\right|} \\
u_{N-1}^{n+1}=\frac{\rho_{N-1, L}\left|\Omega_{N-1, L}\right| u_{N-1}^{a}}{\rho_{N-1, R}\left|\Omega_{N-1, R}\right|+\rho_{N-1, L}\left|\Omega_{N-1, L}\right|}
\end{gathered}
$$


is used at the walls in cells $i=0, N-1$ rather than (4.19). Updating velocity at the wall using (4.19) can result in spurious ringing. Mass is conserved in both water and air to order $\mathcal{O}\left(10^{-12}\right)$. The maximum fluctuation in the volume of the water column is $0.10 \%$.

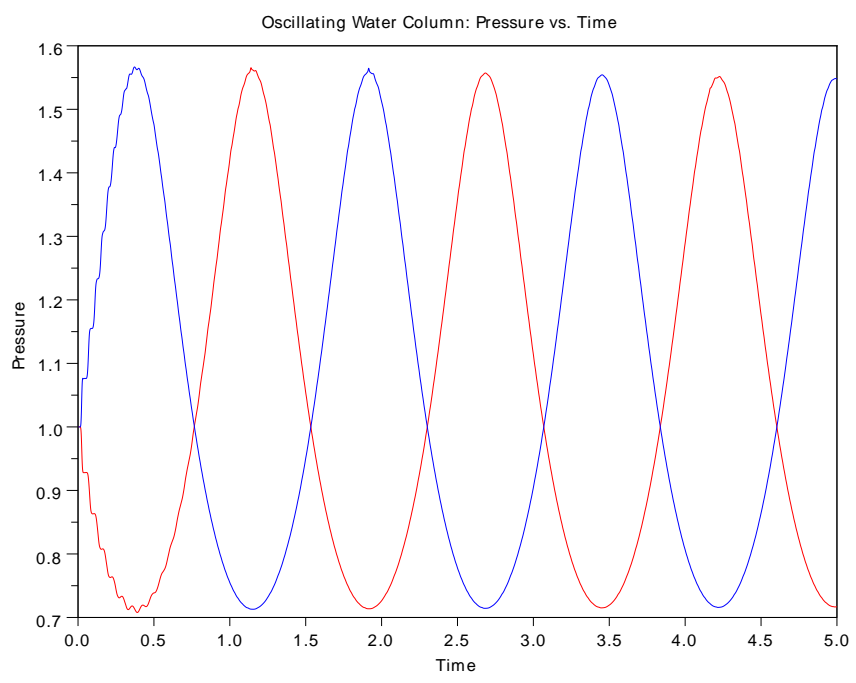

Figure 18: Pressure at the ends of the oscillating water column. The blue line indicates pressure at the right end, red indicates pressure at the left end. A linear MINMOD slope reconstruction for all conserved variables is used.

Results are shown in Figure 18, for resolution $N=160$. Note that the amplitude and frequency of oscillation agree with the results shown in Koren and Kadioglu. This result is novel, in that it does not make the assumption of spatially uniform density in the gas region, or that the gas is adiabatic. Even though both materials are taken to be fully compressible, the total fluctuation of the volume of the water column is less than $\Delta x / 50$. Additionally, there is no mass loss for either material, up to machine precision.

\subsection{D JWL Shock Tube}

A tube contains an inviscid gas governed by the Jones-Wilkins-Lee (JWL) equation of state (6.18) and inviscid water, taken to be governed by the Tait equation of state (6.17), as in Wardlaw [55]. The Tait equation of state makes 
the assumption that water is adiabatic. A lower bound is added to (6.17) in order to account for cavitation in the liquid. If density falls below the cutoff value $\rho_{c}$, then the sound speed is computed using the cutoff density.

$$
p= \begin{cases}B\left(\left(\frac{\rho}{\rho_{0}}\right)^{\gamma}-1\right)+A, & \text { if } \rho>\rho_{c} \\ p_{c}, & \text { otherwise }\end{cases}
$$

$A=1.0 \times 10^{6} \mathrm{~d} / \mathrm{cm}^{2}, B=3.31 \times 10^{6} \mathrm{~d} / \mathrm{cm}^{2}, \gamma=7.15, \rho_{0}=1.0 \mathrm{~g} / \mathrm{cm}^{3}$, $\rho_{c}=1.0-4.225 \times 10^{-5} \mathrm{~g} / \mathrm{cm}^{3}$, and $p_{c}=220.2726 \mathrm{~d} / \mathrm{cm}^{2}$.

The Jones-Wilkins-Lee (JWL) equation of state (6.18) is a model of explosive gas, relating pressure to density and energy.

$$
\begin{gathered}
p=A\left(1-\frac{\omega \rho}{R_{1} \rho_{0}}\right) \exp \left(-R_{1} \frac{\rho_{0}}{\rho}\right)+B\left(1-\frac{\omega \rho}{R_{2} \rho_{0}}\right) \exp \left(-R_{2} \frac{\rho_{0}}{\rho}\right)+\omega \rho e_{\text {int }} \\
\quad \begin{array}{l}
\text { (6.18) } \\
\omega=5.484 \times 10^{12} \mathrm{~d} / \mathrm{cm}^{2}, B=0.09375 \times 10^{12} \mathrm{~d} / \mathrm{cm}^{2}, R_{1}=4.94, R_{2}=1.21,
\end{array} \\
\omega=0.28, \text { and } \rho_{0}=1.63 .
\end{gathered}
$$

The initial conditions in the JWL gas and water are given by (6.19) and (6.20) respectively. The simulation is stopped before the flow interacts with the boundary. Units are in CGS. The acoustic time step (6.1) with $C F L=1 / 2$ was used.

$$
\begin{gathered}
\operatorname{JWL}(x \leq 200): \rho=1.63, e_{i n t}=4.2814 \times 10^{10}, u=0, p=7.81 \times 10^{10}(6 . \\
\operatorname{Water}(x>200): \rho=1.0, e_{i n t}=\mathrm{N} / \mathrm{A}, u=0, p=1.00 \times 10^{6}
\end{gathered}
$$

Results are shown at resolution $N=400$ for comparison with Wardlaw [55]. A slight overshoot at the shock front can be seen. Mass in the liquid and gas regions is conserved to $\mathcal{O}\left(10^{-12}\right)$. A comparison can be made to the shock capturing method of Fedkiw, et. al [18], which eliminates the overshoot at the shock front by extrapolating material to a "ghost cell." 

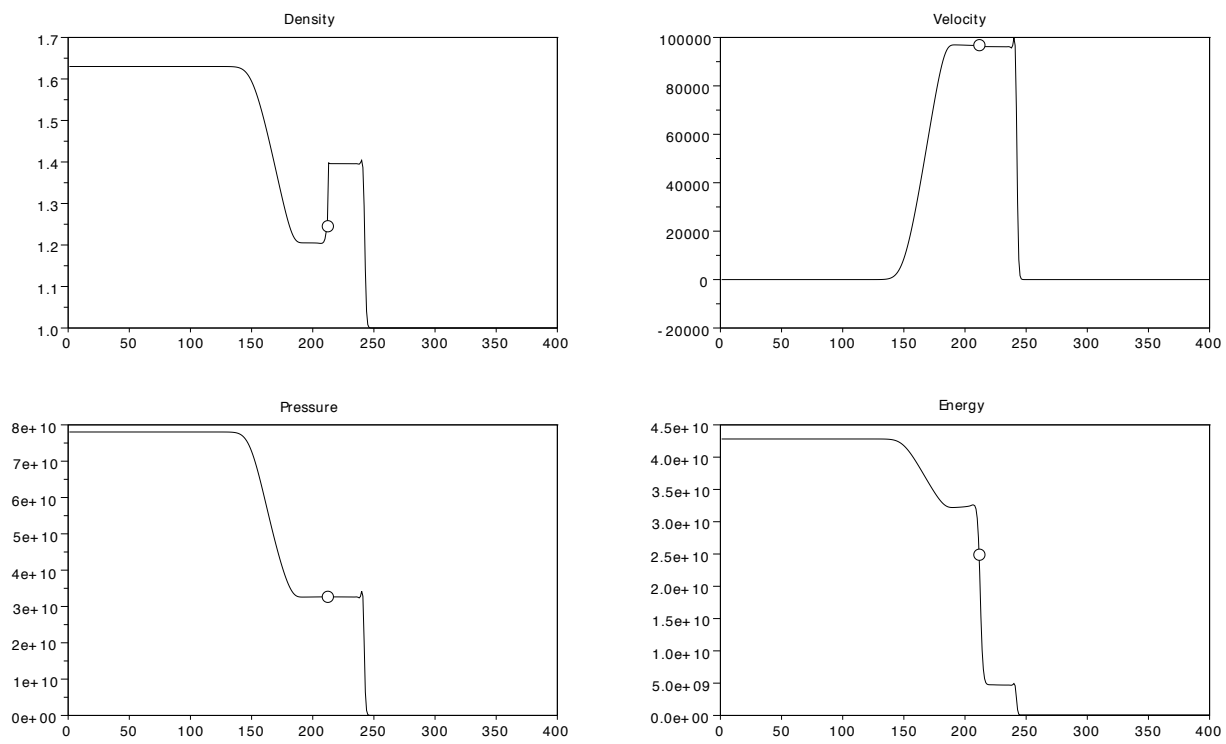

Figure 19: Results for the JWL-Tait shock tube at time $t=0.125$. The material interface is shown as a circle. A linear MINMOD slope reconstruction for all conserved variables is used.

\subsection{Spherical Explosion Shock}

An underwater blast is simulated by initializing a stationary inviscid JWL bubble (6.18) surrounded by inviscid liquid taken to follow the Tait equation of state (6.17), as in Wardlaw [55]. The problem is cast in 1D spherical coordinates. A bubble of JWL gas with radius $16 \mathrm{~cm}$. is initialized at the center of the spherical mesh. Initial conditions for the JWL bubble (6.19) and the surrounding liquid (6.20) are taken the same as the JWL shock tube. The acoustic time step (6.1) with $C F L=1 / 2$ was used.

The pressure difference at the interface results in a right-moving shock, left-moving rarefaction, and right-moving contact discontinuity. Pressure drops in the bubble, until a critical stage is reached, when the difference in pressure between the interface and the center of the bubble causes a leftgoing shock to form. The shock reflects off of the internal wall and reshocks the interface. Euler's equations are cast in spherical coordinates, as in [33]. 


\subsubsection{Spherical Lagrangian Characteristic Tracing}

The ODE that governs characteristic backtracing (3.16) is modified for spher-

ical coordinates. Let $\alpha$ denote the foot of a characteristic, $u_{f}$ denote a face velocity, and $r_{f}$ denote radius at the face of a cell. Then, the departure region for a cell is defined by the solution to (6.21) at time $t=\Delta t$.

$$
\left\{\begin{array}{l}
\frac{d \alpha(t)}{d t}=-\frac{u_{f} r_{f}^{2}}{\alpha^{2}} \\
\alpha(0)=r_{f}
\end{array}\right.
$$

A linear mapping is applied to map points in the departure region $\Omega_{D}$ to points in the target cell, as in Section 1. The coefficient $\mathcal{C}_{r}$, the spherical analogue of $\mathcal{C}(3.3)$, is computed as

$$
\begin{gathered}
\mathcal{C}_{r}=\frac{\Delta r}{\alpha_{i+1 / 2}-\alpha_{i-1 / 2}} . \\
f(r)=\mathcal{C}_{r}\left(r-\alpha_{i-1 / 2}\right)+r_{i-1 / 2}
\end{gathered}
$$

A discretely divergence-free face velocity in spherical coordinates does imply that $\mathcal{C}=1$, i.e. that $\left|\Omega_{D}\right|=|\Omega|$, so a constant field is preserved under a divergence-free face velocity in a single material. This does not hold for multiple materials. Because the mapping from the departure region to the target region is linear, contributions from individual cell may undergo expansion or compression, but the errors cancel in computing the cell-averaged value. If an interface cuts the departure region, the individual liquid and gas regions will feel these effects separately, and the errors will not cancel. Hence, a divergence-free velocity will result in slight compression/expansion at the interface. See Fig. 20. A version of (6.21) can be formulated, with an associated mapping, that has the same departure region and exactly preserves a constant field under a divergence-free face velocity.

$$
\frac{d \alpha(r, t)}{d t}=-\frac{A r+B}{\alpha^{2}}
$$

Mapping points from the departure region to the target cell then requires solving for the roots of a depressed cubic for each point. In 2D, a similar technique in cylindrical coordinates maps a polygonal domain in a departure region to a curved figure in the target region, which introduces errors with the volume and moment computations of the Moment of Fluid method. 

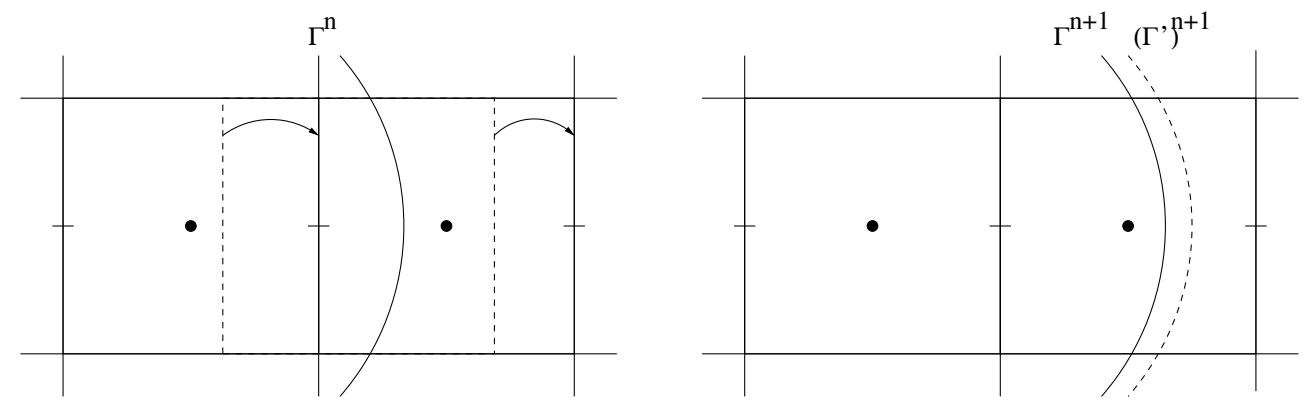

Figure 20: Left: Departure region (dotted box) with interface $\Gamma^{n}$ is mapped to the target cell under a divergence-free face velocity. Right: Reconstructed interface $\Gamma^{n+1}$ according to linear mapping (solid curve), and true interface $\left(\Gamma^{\prime}\right)^{n+1}$ (dotted curve) mapped with the analytic, divergence-free velocity.

Numerical tests for the spherical explosion shock demonstrated very similar behavior in interface location and shock speed between the two methods. As such, the linearity preserving mapping (6.23) is used for problems in spherical coordinates.

\subsubsection{Spherical Explosion Shock in 1D Spherical coordinates}

A comparison is made to the benchmark results in Wardlaw [55], with the simulation terminated at time 5.0E-4 seconds. The domain is taken to be of size $256 \mathrm{~cm}$, so that the external boundary does not interfere with the simulation. The internal boundary condition is taken to be a wall, discretized as in (6.15). State variables at the final time are shown in Fig. 21, with the interface denoted by an open circle. Resolution is $\mathrm{N}=512$ cells.

\begin{tabular}{|c|c|c|c|}
\hline Finest $\Delta x$ & Shock Peak & Bubble Position & Number of Time Steps \\
\hline $1 / 2$ & $1.6446 \mathrm{E}+9$ & 41.067 & 522 \\
\hline $1 / 4$ & $1.7550 \mathrm{E}+9$ & 41.139 & 1054 \\
\hline $1 / 8$ & $1.8279 \mathrm{E}+9$ & 41.181 & 2123 \\
\hline
\end{tabular}

Table 5: Data for the Spherical Explosion Shock in 1D Spherical coordinates (Sec. 6.5), with piecewise constant reconstruction for all conserved quantities, two backward sweeps.

Convergence of the solution at the shock in the gas and liquid regions is shown in Figures 22 - 23. Results are shown for density with resolution 

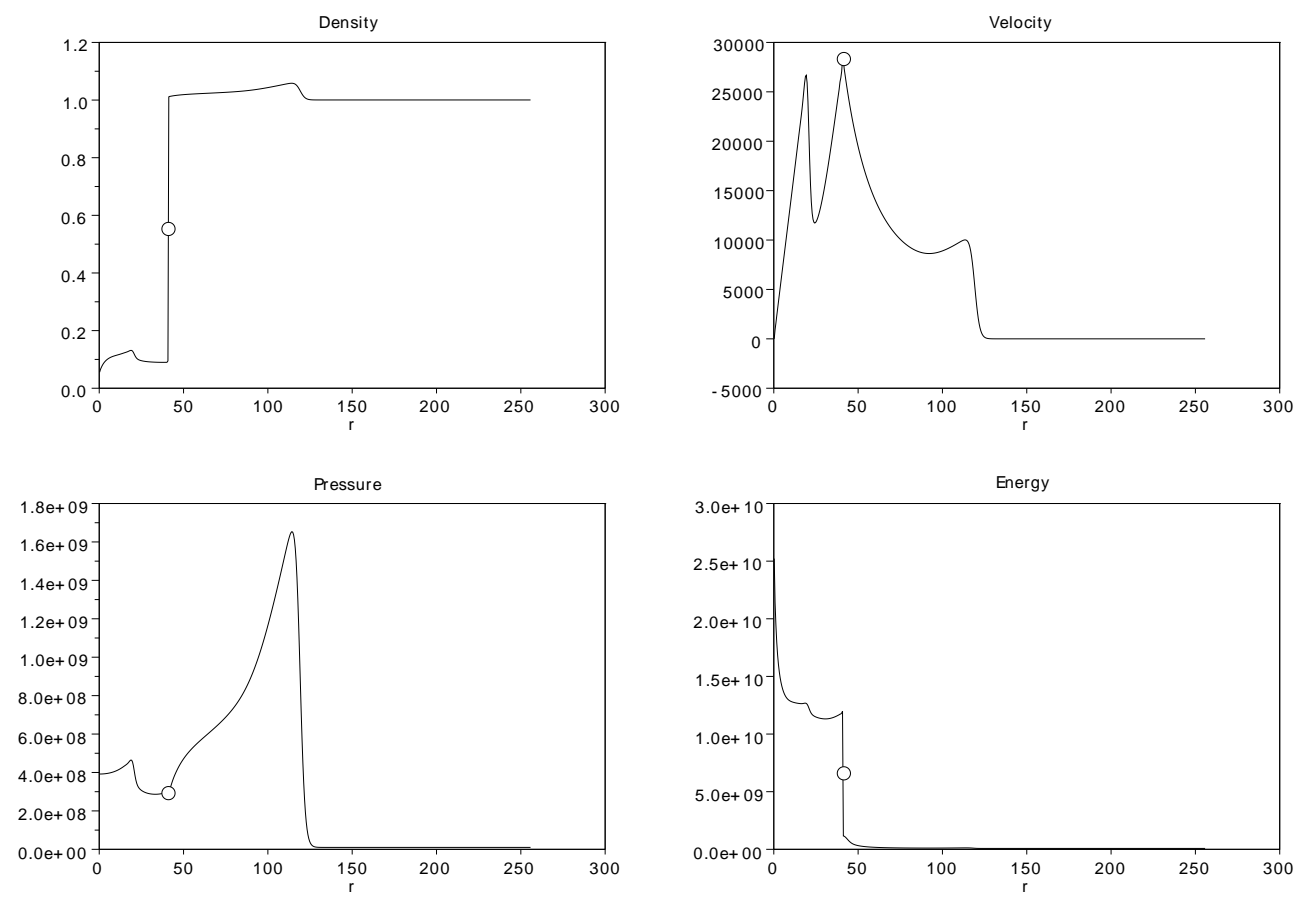

Figure 21: Results for the JWL-Tait spherical explosion shock at time $t=$ $5.0 E-4$ sec. The open circle in each image denotes the location of the material interface. Resolution is $\mathrm{N}=512$ cells. A linear MINMOD slope reconstruction for all conserved variables is used.

\begin{tabular}{|c|c|c|c|}
\hline Finest $\Delta x$ & Shock Peak & Bubble Position & Number of Time Steps \\
\hline $1 / 2$ & $1.6535 \mathrm{e}+9$ & 41.086 & 573 \\
\hline $1 / 4$ & $1.7611 \mathrm{e}+9$ & 41.150 & 1106 \\
\hline $1 / 8$ & $1.8328 \mathrm{e}+9$ & 41.187 & 2177 \\
\hline
\end{tabular}

Table 6: Data for the Spherical Explosion Shock in 1D Spherical coordinates (Sec. 6.5), with linear MINMOD reconstruction for all conserved quantities, two backward sweeps.

varying from $\mathrm{N}=256$ to $\mathrm{N}=4096$. First order convergence is observed in the magnitude and location of the shock from Table 5. The results are consistent with the expected results for a shock capturing method. Our results do not exhibit spurious oscillations at the shock front.

Our results are novel in that we have developed a conservative, shock- 


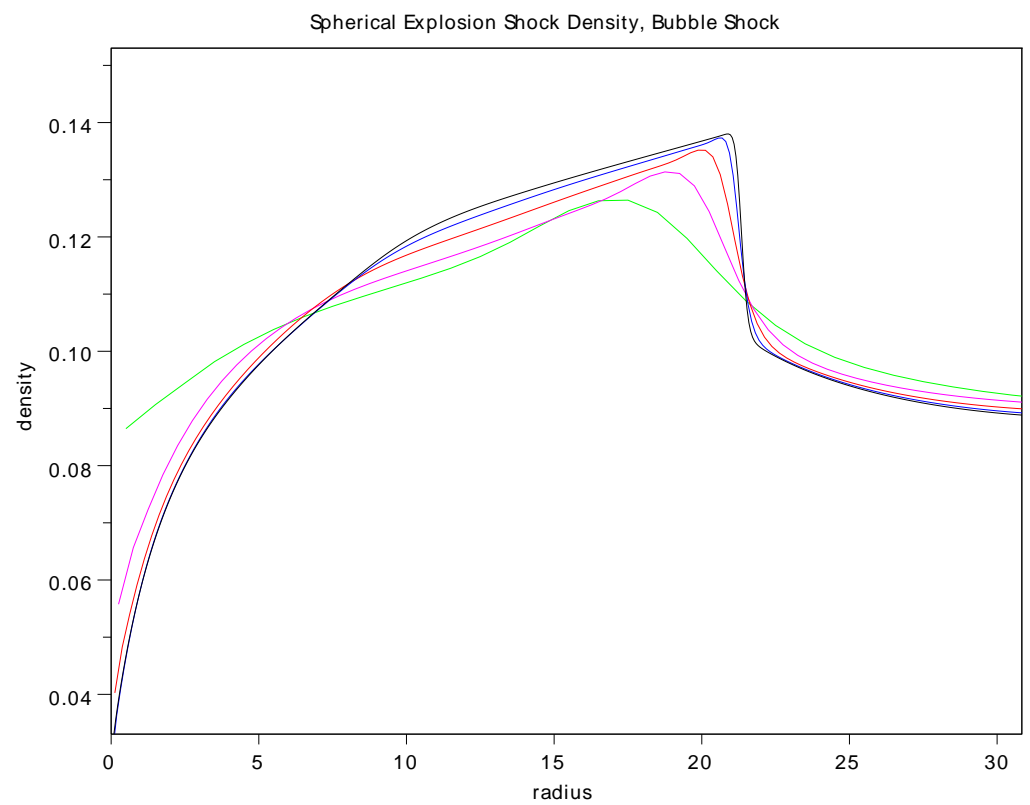

Figure 22: Convergence of density at the shock in the JWL bubble region for the Spherical Explosion Shock at time $t=5.0 E-4$ sec. The colors denote the following resolution: Green $\mathrm{N}=256$, Purple $\mathrm{N}=512$, Red $\mathrm{N}=1024$, Blue $\mathrm{N}=2048$, Black $\mathrm{N}=4096$.

capturing method on a fixed grid to simulate the underwater blast. Unlike Kadioglu [32], the JWL gas is not assumed to be spatially uniform in density and adiabatic. The grid is not deformed with the material interface or fit to the shock, as in Wardlaw [54]. Agreement in location of the material interface, shock location, and magnitude of pressure at the shock front is observed between our results and Wardlaw's benchmark results. Scaling is performed in the computation of characteristics (6.21) and in integration over control volumes to reduce the severity of round-off errors. The relative mass error is $\mathcal{O}\left(10^{-13}\right)$ in the JWL gas and $\mathcal{O}\left(10^{-10}\right)$ in the water. The discrepancy is attributed to round-off error, in that the gas occupies the interior of the domain, where control volumes are much smaller than those toward the edge of the mesh.

Remarks: 


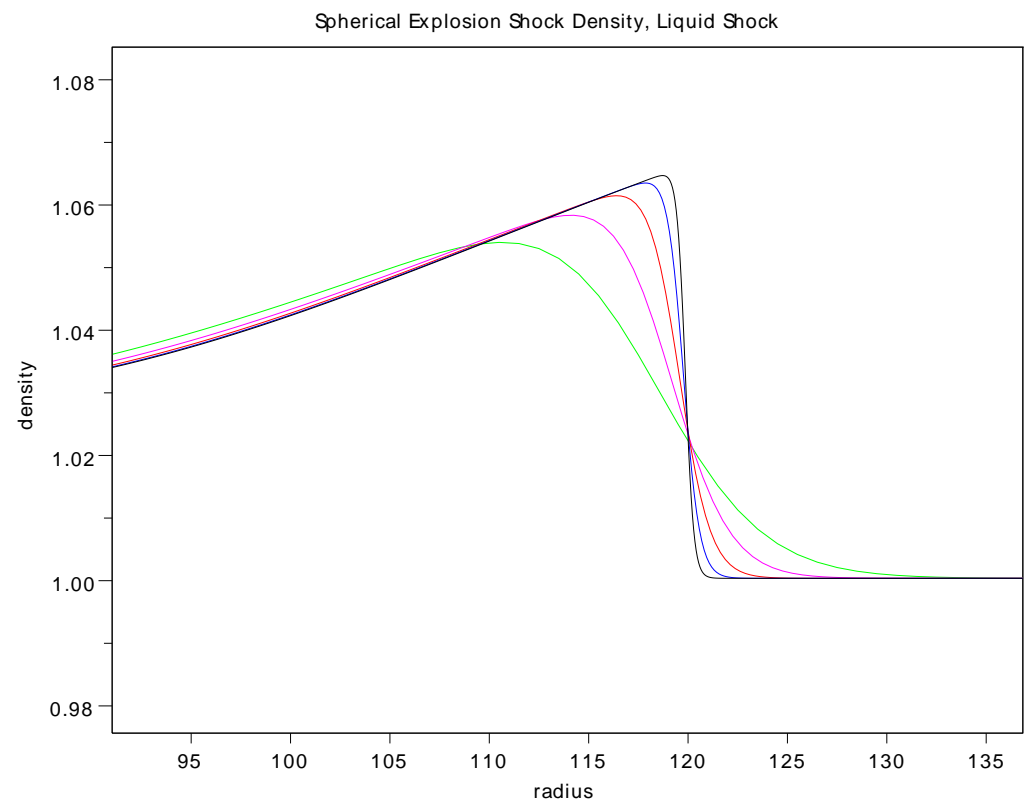

Figure 23: Convergence of density at the shock in the Tait liquid region for the Spherical Explosion Shock at time $t=5.0 E-4 \mathrm{sec}$. The colors denote the following resolution: Green $\mathrm{N}=256$, Purple $\mathrm{N}=512$, Red $\mathrm{N}=1024$, Blue $\mathrm{N}=2048$, Black $\mathrm{N}=4096$.

- Approximate first order convergence in bubble position and magnitude of the pressure shock is observed from Table 5 .

- In Figures 24 and 25 we compare the shock resolution of our algorithm when first order slope reconstruction is used versus MINMOD slope reconstruction (3.10). Since we do not see much of a difference in the results, we prescribe the reconstructed slopes to always be zero for our $2 \mathrm{D}$ and $3 \mathrm{D}$ simulations. 


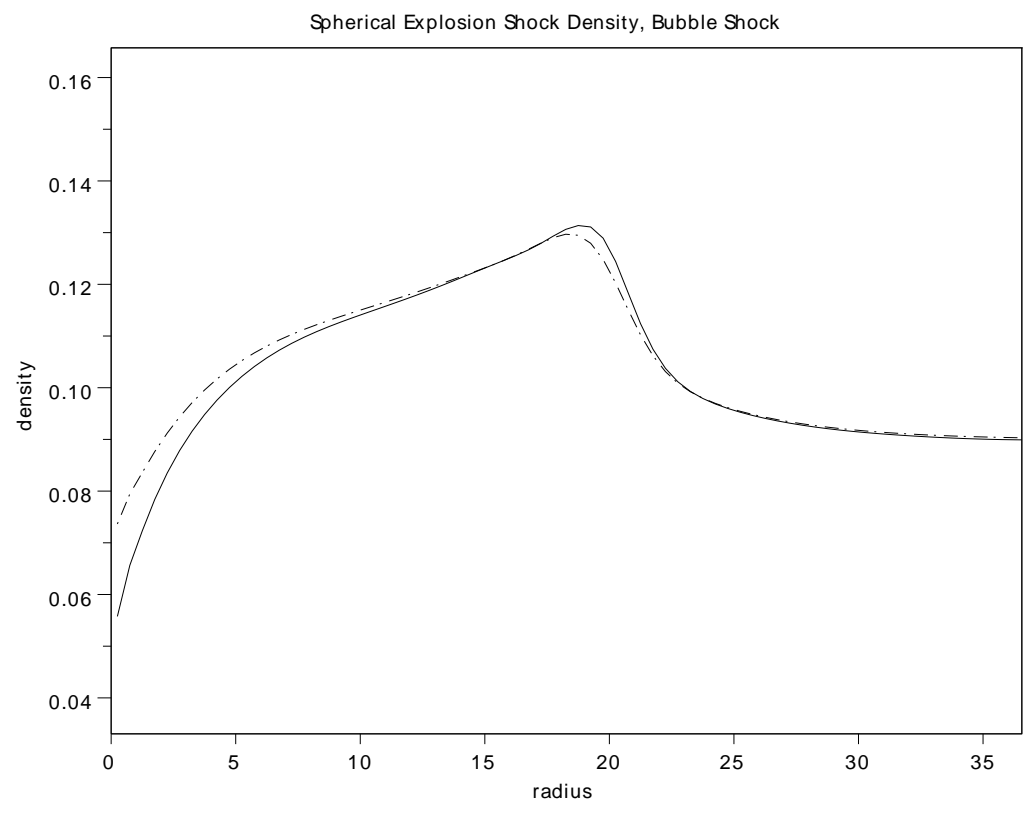

Figure 24: Underwater Spherical Explosion Shock test problem: a comparison between the MINMOD slope reconstruction (solid black line) and piecewise constant reconstruction (dotted line) for capturing the shock wave in the JWL gas region at time $t=5.0 E-4 \mathrm{sec}$. The resolution is $N=512$. 


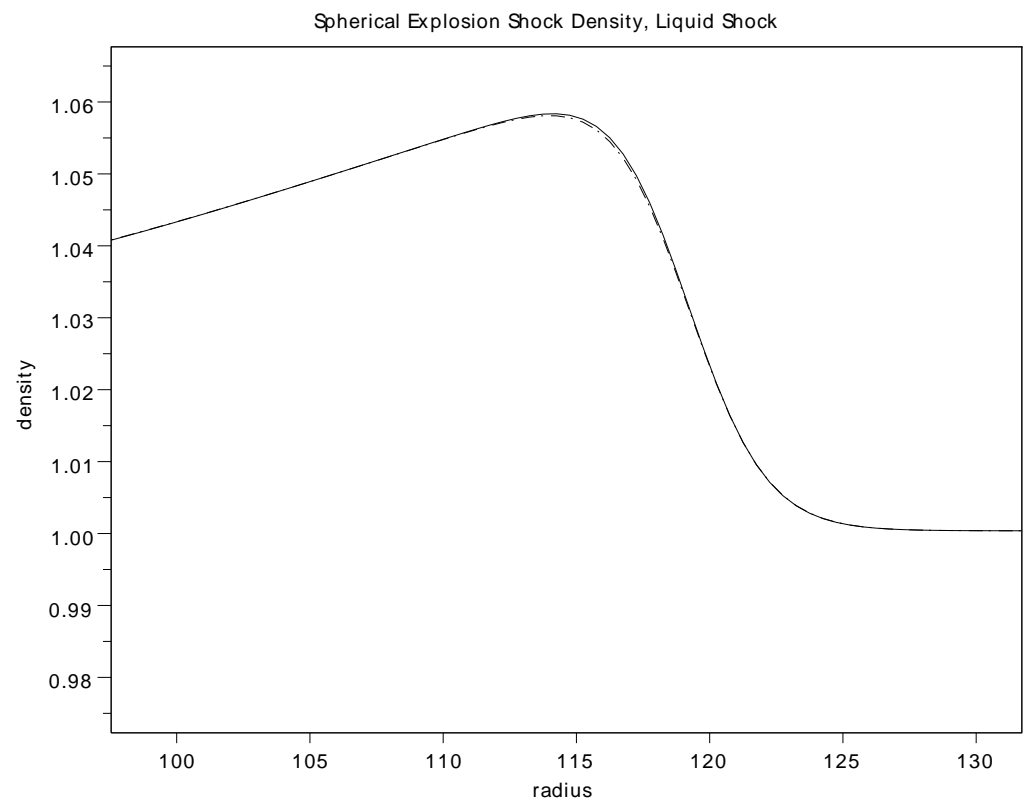

Figure 25: Underwater Spherical Explosion Shock test problem: a comparison between the MINMOD slope reconstruction (solid black line) and piecewise constant reconstruction (dotted line) for capturing the shock wave in the Tait liquid region at time $t=5.0 E-4 \mathrm{sec}$. The resolution is $N=512$. 


\subsection{Spherical Explosion Shock in 3D axisymmetric co- ordinates (RZ)}

The initial conditions in cylindrical coordinates for the JWL explosive material (6.19) and for the surrounding liquid (6.20) are identical to the initial conditions in sections (6.4) and (6.5). Reconstruction of density, momentum, and energy in each cell is taken to be first order (i.e. piecewise constant). An Eulerian-Implicit/Lagrangian-Explicit, directionally-split advection scheme is used, with two advection+pressure correction sweeps per time step. Adaptive Mesh Refinement (AMR) is used to refine the grid; the number of AMR levels used are reported in Table 7. Cells are tagged for AMR when one of the following conditions are met: (1) pressure exceeds 10 times the hydrostatic pressure, (2) $0.1<F^{m}<0.9,(3) F^{m}-1 / 2$ changes sign across adjoining cells.

\begin{tabular}{|c|c|c|}
\hline Base Grid & Levels of AMR & Finest $\Delta x$ \\
\hline $32 \times 32$ & 4 & $1 / 2$ \\
\hline $32 \times 32$ & 5 & $1 / 4$ \\
\hline $64 \times 64$ & 5 & $1 / 8$ \\
\hline
\end{tabular}

Table 7: Mesh resolution for the Spherical Explosion Shock in 3D axisymmetric (RZ) coordinates (Sec. 6.6).

Data for shock peak, interface location, and the number of time steps is shown in Table 8. Comparison can be made to results in 1D spherical coordinates (Table 5), using a piecewise-constant reconstruction for all quantities. Good agreement is seen in both peak pressure shock value and bubble position between the 1D spherical and 2D cylindrical tests. Pressure distribution for the Spherical Explosion shock is shown in Fig. 26. A cross-section of pressure along axes $\theta=0, \pi / 4, \pi / 2$ is shown for the coarsest mesh system $\left(\Delta x^{\text {fine }}=1 / 2\right)$ in Figure 27 which illustrates that our method preserves radial symmetry for this problem. The cross-section plots for the two successive finer mesh systems are practically indistinguishable from each other. 


\begin{tabular}{|c|c|c|c|}
\hline Finest $\Delta x$ & Shock Peak & Bubble Position & Number of Time Steps \\
\hline $1 / 2$ & $1.637 \mathrm{E}+9$ & 41.137 & 573 \\
\hline $1 / 4$ & $1.746 \mathrm{E}+9$ & 41.203 & 1105 \\
\hline $1 / 8$ & $1.818 \mathrm{E}+9$ & 41.235 & 2174 \\
\hline
\end{tabular}

Table 8: Data for the Spherical Explosion Shock in 3D axisymmetric (RZ) coordinates (Sec. 6.6), with first order reconstruction, EI-LE advection. Compare with Table 5.

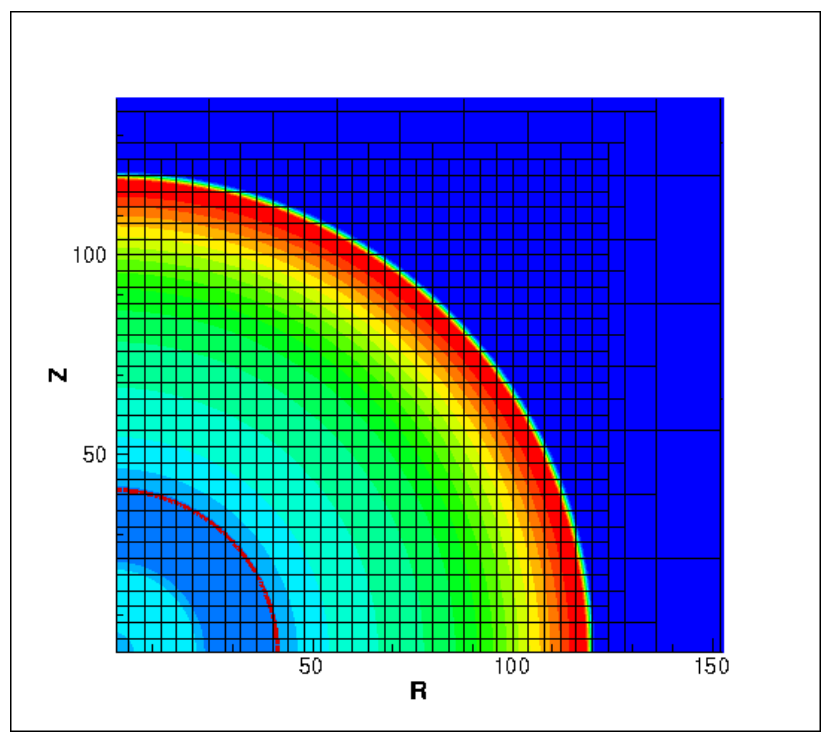

Figure 26: Pressure for the Spherical Explosion Shock at time $t=0.5 \mathrm{~ms}$ in 3D axisymmetric (RZ) coordinates on a fine mesh. Base resolution is $64 \times 64$ with 5 levels of AMR. 


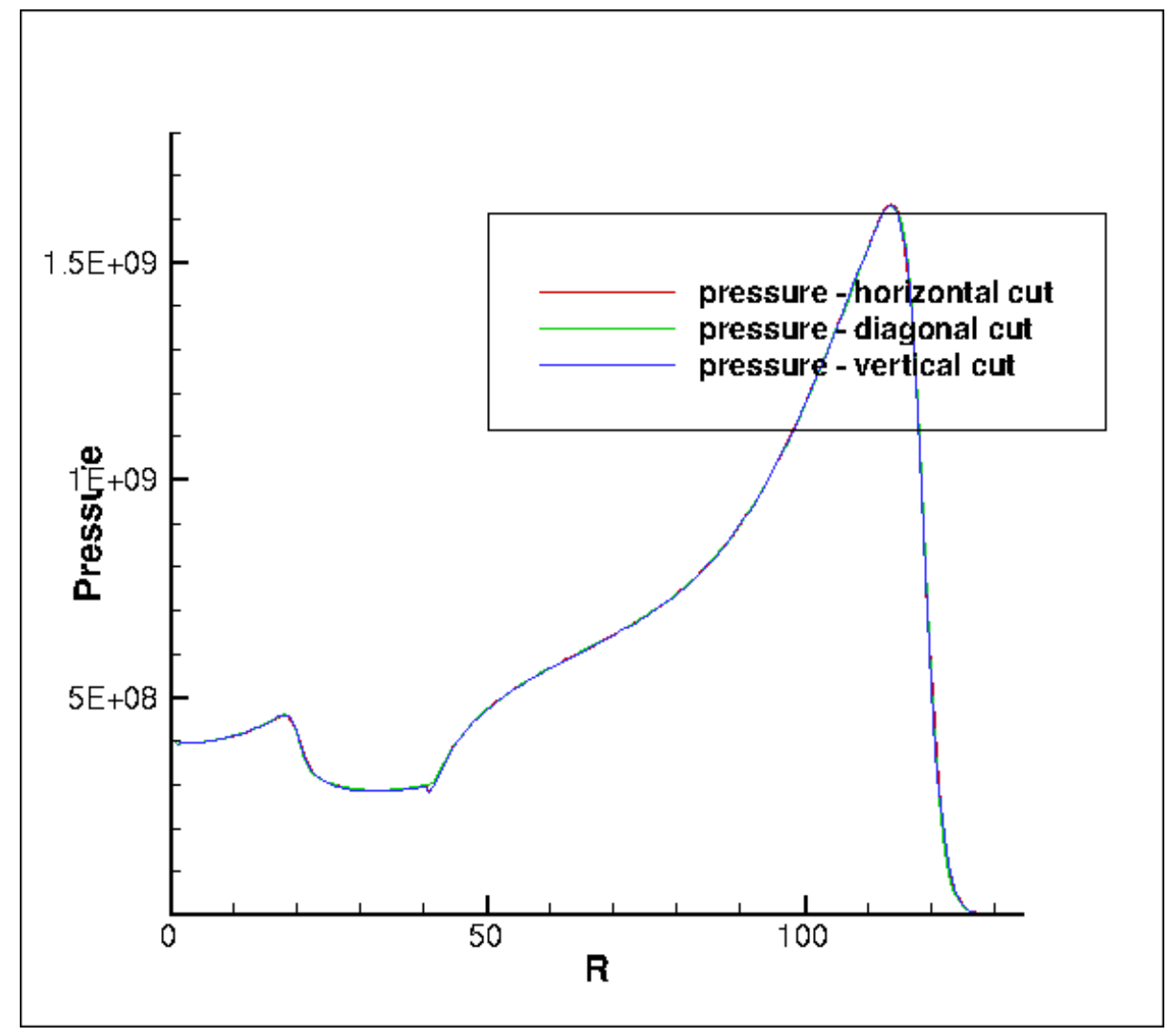

Figure 27: Pressure cross-sections for the Spherical Explosion Shock at time $t=0.5 \mathrm{~ms}$ in $3 \mathrm{D}$ axisymmetric (RZ) coordinates on a coarse mesh. Crosssections are taken at $\theta=0, \pi / 4, \pi / 2$. Base resolution is $32 \times 32$ with 4 levels of AMR.

\subsection{Bubble Jetting}

A bubble of gas governed by the JWL equation of state (6.18) is initialized below a fixed plate submerged in water which is governed by the Tait equation of state (6.17). The coefficients for the JWL equation of state in this test problem are different from those used for the spherical underwater explosion test problems; here the JWL coefficients are:

$A=3.71 E+12, \quad B=0.03231 E+12, \omega=0.3, \quad R_{1}=4.15, \quad R_{2}=0.95, \quad \rho_{0}=1.63 \mathrm{~g} / \mathrm{cm}^{3}$. 
The initial configuration for the explosive and flat plate is illustrated in Figure 28. The initial conditions in the JWL bubble are (6.25):

JWL gas: $\rho=1.63, e_{\text {int }}=4.2945 E+10, \boldsymbol{u}=0, \quad p=8.3837 E+10(6.25)$ The center of the explosive bubble is initialized at a depth of $98.5 \mathrm{~m}$, with radius $1.36 \mathrm{~cm}$. The plate is located $13 \mathrm{~cm}$ above the center of the explosive bubble, with radius $88.9 \mathrm{~cm}$ and thickness $2.54 \mathrm{~cm}$. Static water density,

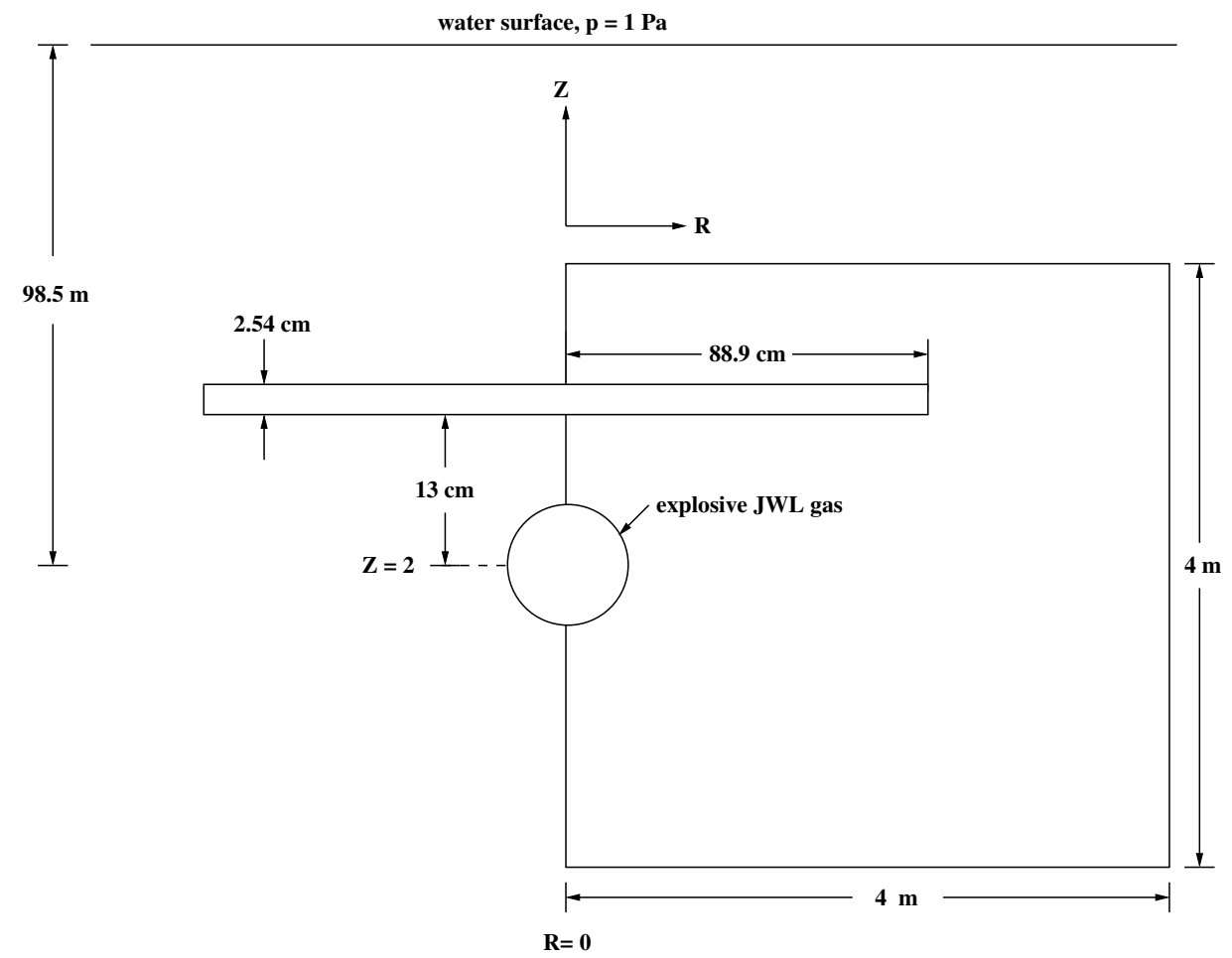

Figure 28: Dimensions and set-up for underwater explosion problem in the vicinity of a fixed flat plate. The computational grid is an adaptive 3D axisymmetric (RZ) grid.

$\rho^{\text {static }}$, is initialized to vary linearly with depth, with $\rho^{\text {static }}=1$ at the free surface and $\rho^{\text {static }}=1.003908$ at the bottom of the domain. Acceleration due to gravity, $g$, is taken to exactly counteract the pressure gradient (6.26), such that the system without the explosive bubble would be stationary. The acceleration due to gravity at cell faces is calculated according to (6.27) for 
the pressure solve. Note that this implies $g$ is not identically $981 \mathrm{~cm} / \mathrm{s}^{2}$, but that the liquid medium is in equilibrium.

$$
\begin{gathered}
g=\frac{\nabla p}{\rho^{\text {static }}} \\
g_{k+1 / 2}=\frac{p\left(\rho_{k+1}^{\text {static }}\right)-p\left(\rho_{k}^{\text {static }}\right)}{\Delta z\left(\frac{\rho_{k+1}^{\text {static }}+\rho_{k}^{\text {static }}}{2}\right)}
\end{gathered}
$$

Water density is specified as a function of depth (6.28). Here $\rho_{d}=1.003908$ is the water density at the depth of the explosive bubble; $h=200 \mathrm{~m}$ is the distance between the "sea floor" and the free surface; and $k$ is the distance from the floor to the center of the bubble.

$$
\rho(y)=1+\left(\rho_{d}-1\right)(h+k-y) / h
$$

The problem is cast in 3D axisymmetric coordinates (RZ). An EI-LE, directionally-split advection scheme is used, with two advection+pressure correction sweeps per time step. Adaptive Mesh Refinement (AMR) is used to refine the grid according to Table 9. Cells are tagged for AMR at the plate, at the bubble-water interface, and when pressure exceeds 10 times the hydrostatic pressure. The domain is taken to be $400 \times 400 \mathrm{~cm}^{2}$, centered in height at the center of the bubble. The plate is aligned to the mesh. The simulation is terminated at $t=15 \mathrm{~ms}$.

Results for pressure and interface location are shown at six different times in Figure 29. The shock wave is shown interacting with the plate, reflecting, and interacting with the bubble. This figure also shows the JWL explosive bubble region growing and then collapsing at the plate. Due to the limited mobility of the liquid inbetween the plate and the bubble, a jet of liquid penetrates the JWL bubble from the opposite side causing the bubble to transform into a toroidal shape. Figures 30 - 31 show the evolution of the bubble's effective radius (6.29) over time for small and large time steps, respectively. The small time stepping scheme (6.1) uses the stringent acoustic time step constraint with $C F L=1 / 2$. The large time stepping scheme uses the standard, acoustic time step formulation until time $t=0.67 \mathrm{~ms}$, before which the initial shock wave is interacting with the plate. From time $t=0.67-15 \mathrm{~ms}$, the much more lenient advective time step constraint (6.2) with $C F L=1 / 4$ is taken. Both Figures 30 (small time stepping scheme) and 31 (large time stepping scheme) demonstrate convergence under grid 
refinement and demonstrate that the results using the large time-stepping scheme converge to the same results when using the small time-stepping scheme.

The large time stepping scheme results in similar bubble behavior, while reducing runtime by nearly a factor of five. Runtimes for the small time stepping procedure at various mesh resolutions are shown in Table 10. Runtimes for the large time stepping procedure are shown in Table 11.

$$
r_{e f f}=\left(\frac{3 V}{4 \pi}\right)^{1 / 3}
$$

\begin{tabular}{|c|c|}
\hline Base Grid & Levels of AMR \\
\hline $128 \times 128$ & 2 \\
\hline $128 \times 128$ & 3 \\
\hline $128 \times 128$ & 4 \\
\hline
\end{tabular}

Table 9: Mesh resolution for the Bubble Jetting problem in 3D axisymmetric (RZ) coordinates (Sec. 6.7).

\begin{tabular}{|c|c|c|}
\hline AMR Levels & Iterations & Runtime (sec) \\
\hline 2 & 6215 & 39684 \\
\hline 3 & 12410 & 134138 \\
\hline 4 & 25347 & 448426 \\
\hline
\end{tabular}

Table 10: Iterations and runtimes for the Bubble Jetting problem with small time stepping (6.1).

\begin{tabular}{|c|c|c|}
\hline AMR Levels & Iterations & Runtime (sec) \\
\hline 2 & 723 & 7884 \\
\hline 3 & 1542 & 25551 \\
\hline 4 & 3423 & 95343 \\
\hline
\end{tabular}

Table 11: Iterations and runtimes for the Bubble Jetting problem with large time stepping for $t>0.67 m s$ (6.2).

Additionally, agreement in maximum bubble radius and collapse time is seen between the converged results for the small and large $\Delta t$. This indicates 
that the semi-implicit pressure formulation allows for a larger time step while retaining accuracy. Volume $V$ is taken as the volume of JWL gas from the Moment of Fluid interface reconstruction.

As stated in [55], there is no reliable experimental benchmark for verification of this problem, due to deflection and motion in the plate during the experiment. Results can be compared to $[32,55]$ with qualitative agreement. Maximum effective radius is approximately $18 \mathrm{~cm}$, with collapse occurring at approximately $t=13.5 \mathrm{~ms}$. These results are novel in that mass is conserved in both materials, and spatial variation of density and pressure is allowed in the JWL gas. [32] makes a constant density/pressure assumption in the gas phase. The semi-implicit pressure formulation allows for much larger time steps than taken in [55], drastically reducing runtime while obtaining qualitatively similar interface dynamics. 

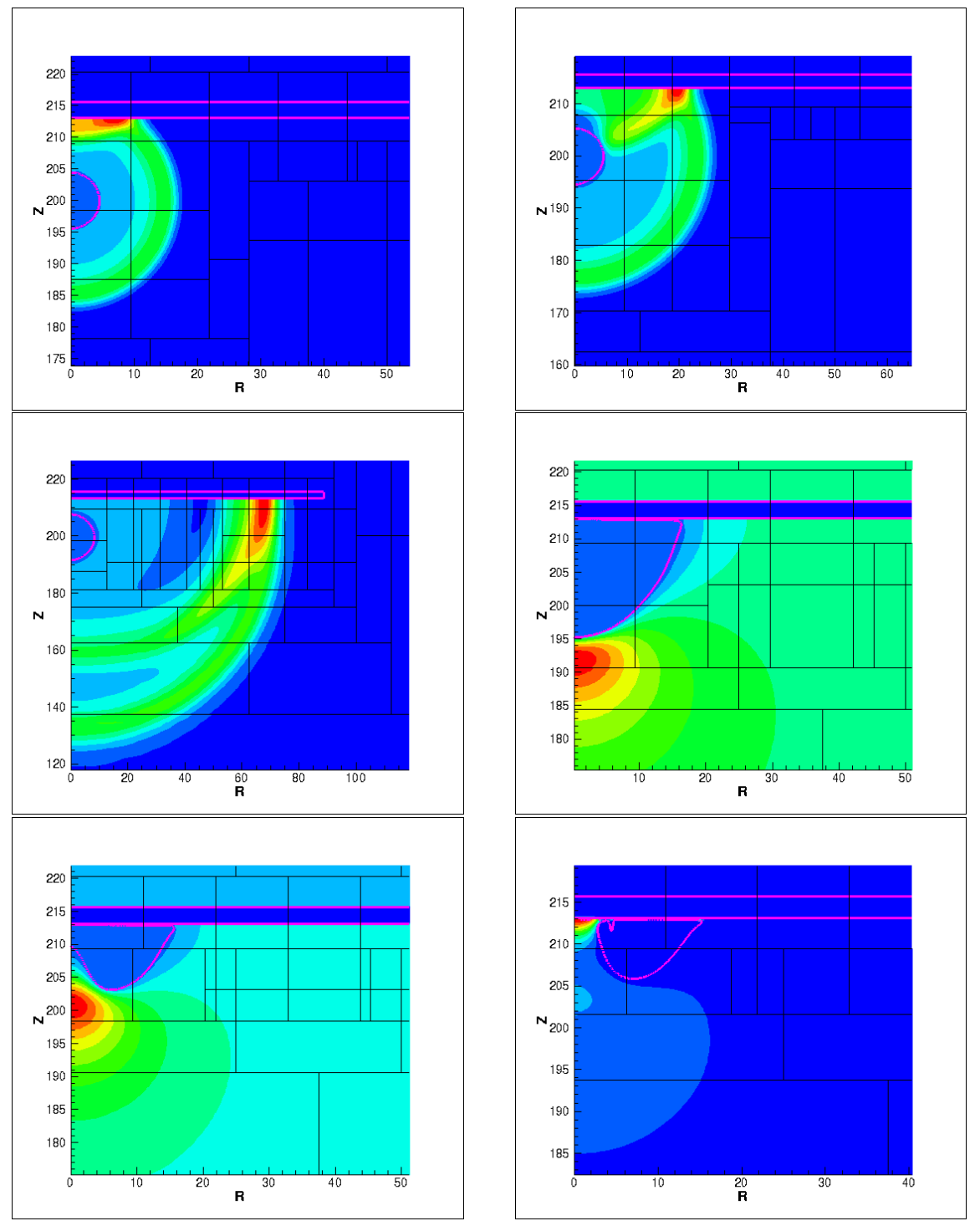

Figure 29: Pressure contours for the simulation of an underwater explosion near a fixed flat plate at times (left to right, top to bottom) $t=0.08 \mathrm{~ms}$, $t=0.14 \mathrm{~ms}, t=0.43 \mathrm{~ms}, t=11.7 \mathrm{~ms}, t=12.4 \mathrm{~ms}$, and $t=12.8 \mathrm{~ms}$. An initial shock wave reflects from the plate and interacts with the bubble. At later times the bubble expands and collapses against the plate, transforming the bubble into a toroidal shape. The bubble interface and plate are shown in pink. An adaptive, 3D axisymmetric (RZ) grid is used. The base grid size is $128 \times 128$ and the effective fine grid resolution is $2048 \times 2048$. 


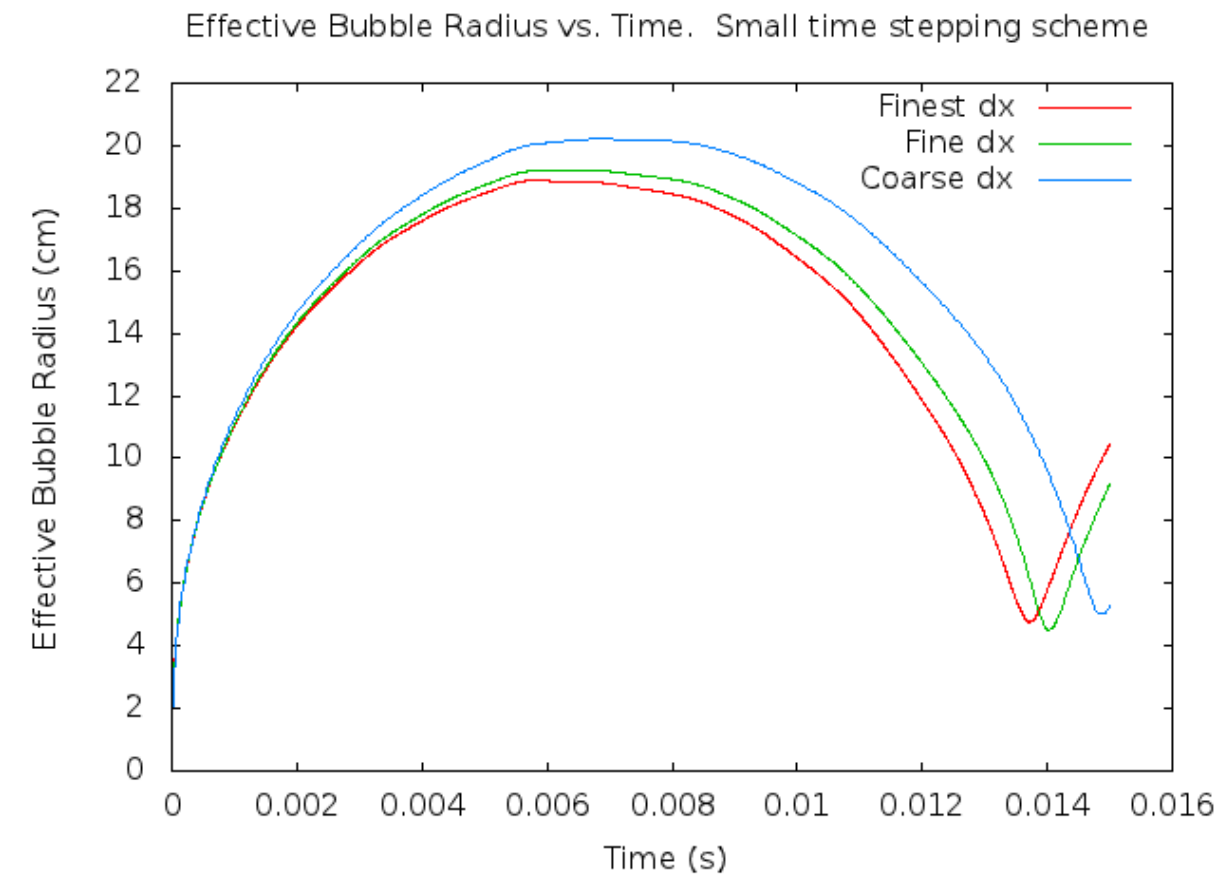

Figure 30: Effective bubble radius for the bubble jetting problem, with the coarsest mesh resolution in blue, finer mesh resolution in green, and finest in red, from Table 9. Uses small time stepping scheme 


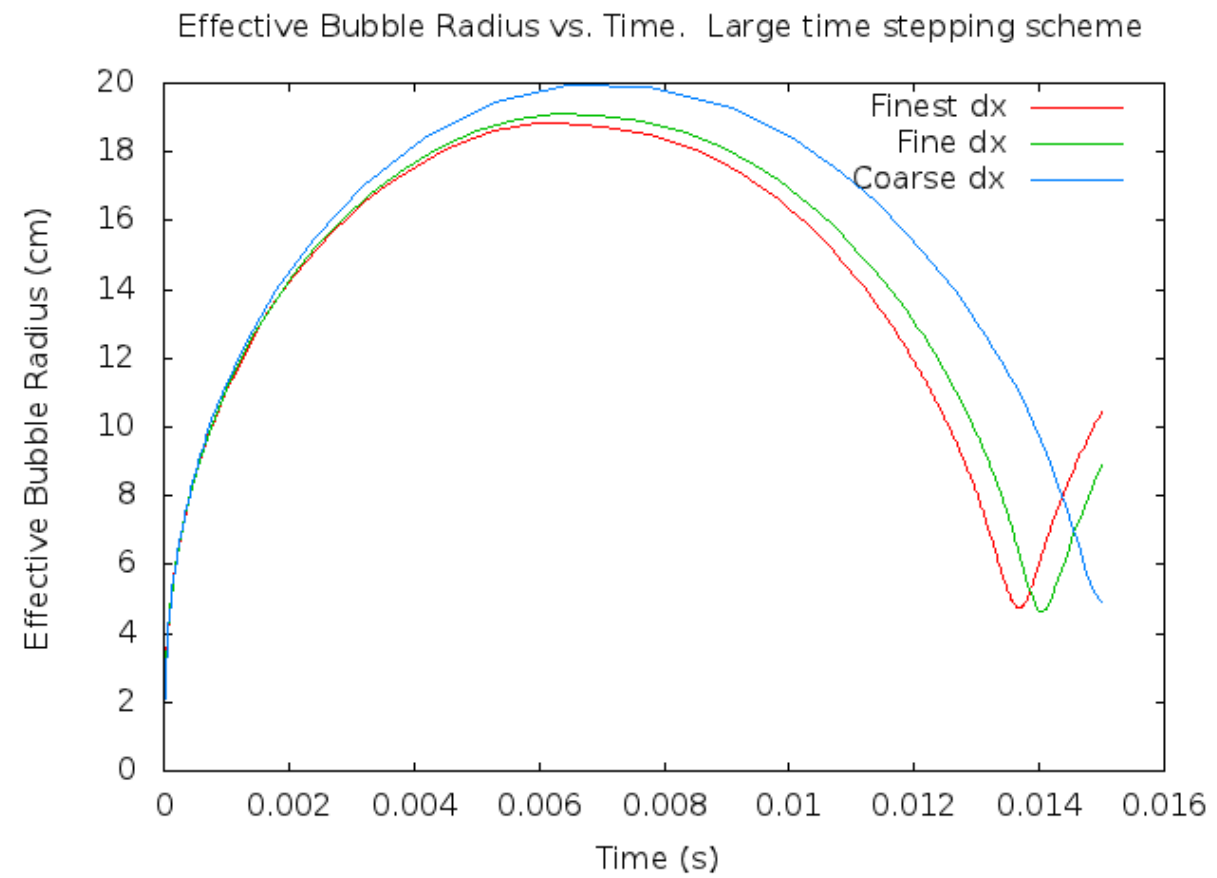

Figure 31: Effective bubble radius for the bubble jetting problem, with the coarsest mesh resolution in blue, finer mesh resolution in green, and finest in red, from Table 9. Uses large time stepping scheme. 


\subsection{Axisymmetric Gas bubble rising in liquid}

We compare results predicted from our 3D axisymmetric (RZ) compressible multiphase flow algorithm with experimental results reported by Hnat and Buckmaster[26] (bubble A page 189) for a gas bubble rising in liquid. The density ratio is $\rho_{l} / \rho_{g}=714$ and the viscosity ratio is $\mu_{l} / \mu_{g}=6667$. We nondimensionalize by the bubble diameter $D=12.15 \mathrm{~mm}$ and the experimentally observed bubble rise speed, $U=21.5 \mathrm{~cm} / \mathrm{s}$. The experimental physical properties are $\mu_{L}=1.18 P, \sigma=32.2 d y n / \mathrm{cm}$, and $\rho_{L}=0.8755 \mathrm{~g} / \mathrm{cm}^{3}$. The Reynolds number becomes $R e=\rho D U / \mu=19.4$, the Weber number becomes $W e=\rho D U^{2} / \sigma=15.27$ and the Froude number is $\mathrm{Fr}=U^{2} /(g D)=0.388$.

[52] simulated this problem assuming both the liquid and gas were incompressible fluids. [1] simulated this problem assuming the liquid was incompressible and the gas was compressible obeying a simplified adiabatic equation of state. For our test we assume both the gas and liquid are compressible materials. The liquid is taken to obey the Tait equation of state (6.17). We remark that $\rho$ corresponds to the dimensionless density in (6.17) and we assume that the dimensionless values for $A$ and $B$ in (6.17) correspond to the dimensional values too. The gas is taken to obey the ideal gas equation of state (6.6).

The computational domain is set to $0<r<8$ and $0<z<24$. The computational grid is a dynamic block structured adaptive grid; the coarsest level is a $128 \times 384$ grid, and there are two levels of refinement making the effective fine grid resolution $512 \times 1536$. We prescribe reflecting boundary conditions at $r=0$ and we prescribe outflow boundary conditions at the other three boundaries. The pressure is prescribed to be the liquid hydrostatic pressure and the velocity satisfies the free boundary condition $\nabla \boldsymbol{u} \cdot \boldsymbol{n}=0$ at outflow boundaries. The dimensionless liquid density is initialized similarly as in the bubble jetting problem from section 6.7. The initial liquid density is given a linear profile in which $\rho_{\text {liquid }}=1$ at $z=24$ and $\rho_{\text {liquid }}=1.0000000026120688$ at $z=0$. The density at $z=0$ was determined so that $p\left(\rho_{\text {liquid }}(z=0)\right)=$ $10^{6}+24 / F r$. The bubble density and internal energy are initially uniform in the bubble and satisfy $p\left(\rho_{\text {gas }}, e_{\text {gas }}\right)=p\left(\rho_{\text {liquid }}\left(z=z_{\text {bubble }}\right)\right)$ where $z_{\text {bubble }}=1.0$ is the initial vertical position of the bubble.

The gravity force is determined in the same manner as for the bubble jetting problem (6.27). At $r=0$ we prescribe symmetric boundary conditions and at $r=8$ we prescribe that $p=p\left(\rho_{\text {liquid }}(z)\right)$ where $\rho_{\text {liquid }}(z)$ is the initial liquid density. 
The initial bubble is a sphere centered at $(0,1.0)$ with radius 0.5 . Results at dimensionless time $t=20.0$ computed using our compressible moment of fluid algorithm are shown in Figure 32. Here we have juxtaposed the computed bubble shape with the experimental bubble shape. In Figure 33, we plot the computed bubble position versus time together with the linear least squares best fit for $4 \leq t \leq 20$ : $y=1.020 t+0.846$. The two curves almost completely overlap. The expected dimensionless rise speed is 1 and the computed dimensionless rise speed fluctuates very little about 1.02. The mass of the bubble fluctuates only in the 6th significant digit. The volume of the bubble fluctuates by at most $0.6 \%$. The fact that the mass of the bubble fluctuates very little is expected because the main source of error for the bubble mass is due to the truncation of the volume fraction to zero or one according to (3.5). The small fluctuation in volume indicates that our algorithm maintains material discontinuities as sharp and is further evidence of the asymptotically preserving property of our algorithm. The time step that we used for this test was

$$
\Delta t=C F L \frac{\Delta x}{u_{\text {tension }}}
$$

where $C F L=1 / 2$ and $u_{\text {tension }}=2.46 . u_{\text {tension }}$ is the characteristic speed of capillary waves associated with $\Delta x$. If we had determined $\Delta t$ using the acoustic time step constraint associated with the liquid, then $\Delta t$ would have been about 5 orders of magnitude smaller. The acoustic time step constraint associated with the gas would have been about 4 orders of magnitude smaller. 


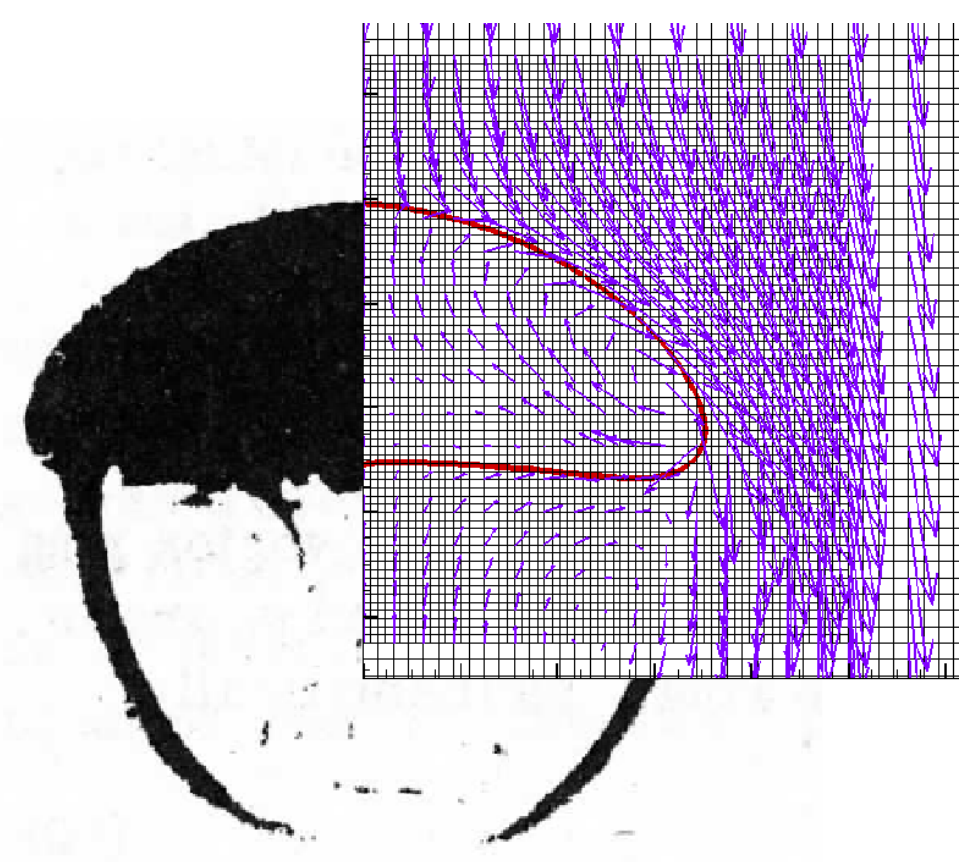

Figure 32: Simulation of a compressible gas bubble (dimensionless time $t=20$ ) rising in a compressible liquid using our compressible multimaterial moment of fluid method. The effective fine grid resolution is $512 \times 1536$. The density ratio is $1: 714$. The physical properties, initial bubble dimensions, and bubble on the left, correspond to cap bubble A in the paper by Hnat and Buckmaster[26]. 1.02 is subtracted from the vertical velocity in the illustration. 


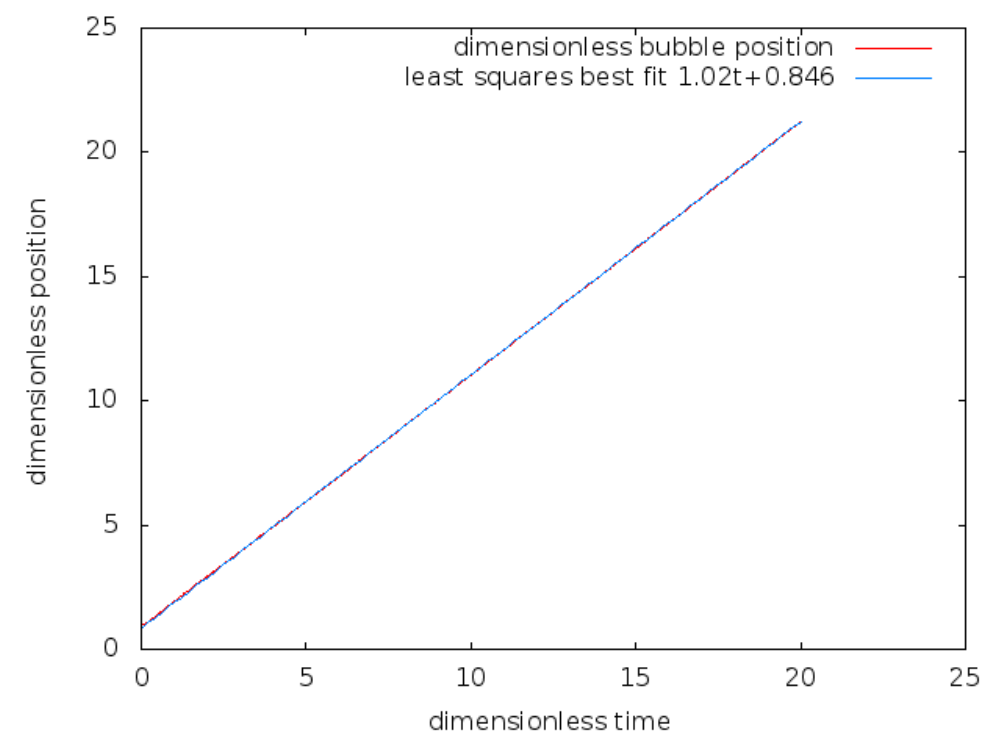

Figure 33: Plot of dimensionless bubble position versus dimensionless time together with the least squares best fit $4 \leq t \leq 20: y=1.020 t+0.846$. The simulation domain is $8 \times 24$. The expected slope is 1.0. The effective fine grid resolution is $512 \times 1536$. The density ratio is $1: 714$. The physical properties and bubble dimensions correspond to cap bubble A in the paper by Hnat and Buckmaster[26]. 


\subsection{Laminar Jet in a Crossflow}

A laminar liquid jet issues from a circular nozzle into a domain with significant gas crossflow. The momentum exchange from the gas to the liquid causes an angular deflection of the jet, while surface instabilities eventually determine its breakup into drops. This problem is cast in 3D Cartesian coordinates. The domain $\Omega$ is defined as in (6.30), with units given in $\mathrm{cm}$.

$$
\Omega=(x, y, z) \in[0,2] \times[0,1] \times[0,2]
$$

The liquid nozzle is located at $\vec{x}=(0.6,0,0) \mathrm{cm}$ with radius $r=0.024 \mathrm{~cm}$. The liquid inflow velocity is $210 \mathrm{~cm} / \mathrm{s}$. Gas inflow at $x=0$ has velocity $2060 \mathrm{~cm} / \mathrm{s}$. Outflow conditions are applied at $x=2, y=1$, and $z=2$. A reflecting wall is specified at $y=0$.

The simulation is run as both compressible and incompressible flow. In the compressible simulation, a directionally split alternating EI-LE method (6.31) similar to the methods described in $[45,7]$ is used. Eulerian Implicit (EI) refers to backward tracing advection which maps material from a departure region. Lagrangian Explicit (LE) refers to forward tracing advection, which maps material to a target region. The nomenclature $X(E I)$ refers to a step of backward traced advection in the $\mathrm{x}$-direction. The liquid is taken to be governed by the Tait equation of state (6.17), and the gas is governed by the ideal gas equation of state (6.6). The time step for this test problem is determined using the advective time step constraint (6.2) with $C F L=0.25$.

$$
X(E I)-Y(L E)-Z(E I)-Z(L E)-Y(E I)-X(L E)
$$

For the incompressible simulation, the directionally split method of Weymouth and Yue [57] is used (6.32), in which material properties are integrated along backwards traced characteristics in each cardinal direction. The advective time step constraint (6.2) with $C F L=1 / 6$ determines $\Delta t$ for the incompressible simulation.

$$
X(E I)-Y(E I)-Z(E I)
$$

The surface tension coefficient is $\sigma=24$ dyne $/ \mathrm{cm}$, the viscosity of liquid and gas are $\mu_{L}=0.0099 \mathrm{~g} /(\mathrm{cms})$ and $\mu_{G}=0.0002 \mathrm{~g} /(\mathrm{cms})$ respectively, the base density of liquid and gas are $\rho_{L}=0.77 \mathrm{~g} / \mathrm{cm}^{3}$ and $\rho_{G}=0.0012 \mathrm{~g} / \mathrm{cm}^{3}$ respectively, and the initial temperature is $290.36 \mathrm{~K}$. 
Data presented in Figure 34 represents the time maximal height, $f(x)$,

$$
f(x)=\max _{0.012 \leq t \leq 0.022} h(x, y=0, t),
$$

of the interface profile along the $y=0$ plane. The function $h(x, y, t)$ represents the highest extent of liquid in the $z$ direction at point $(x, y)$ at time $t$.

Figure 34 shows the maximal height of the incompressible and compressible simulations together with the experimental data from Brown and McDonell [10]. Close agreement is seen between both simulations and the experimental data. Figures 35 and 36 show the $1 / 2$ contour of the volume fraction function for incompressible and compressible flow simulations at representative times. Both methods capture the deflection of the liquid jet and breakup into droplets. Since the height of the liquid jet as a function of time behaves sporadically far downstream of the jet nozzle, we were only able to select comparison times that were in close proximity to each other while also illustrating the representative structure of the deflected jet as it broke up downstream of the nozzle. 


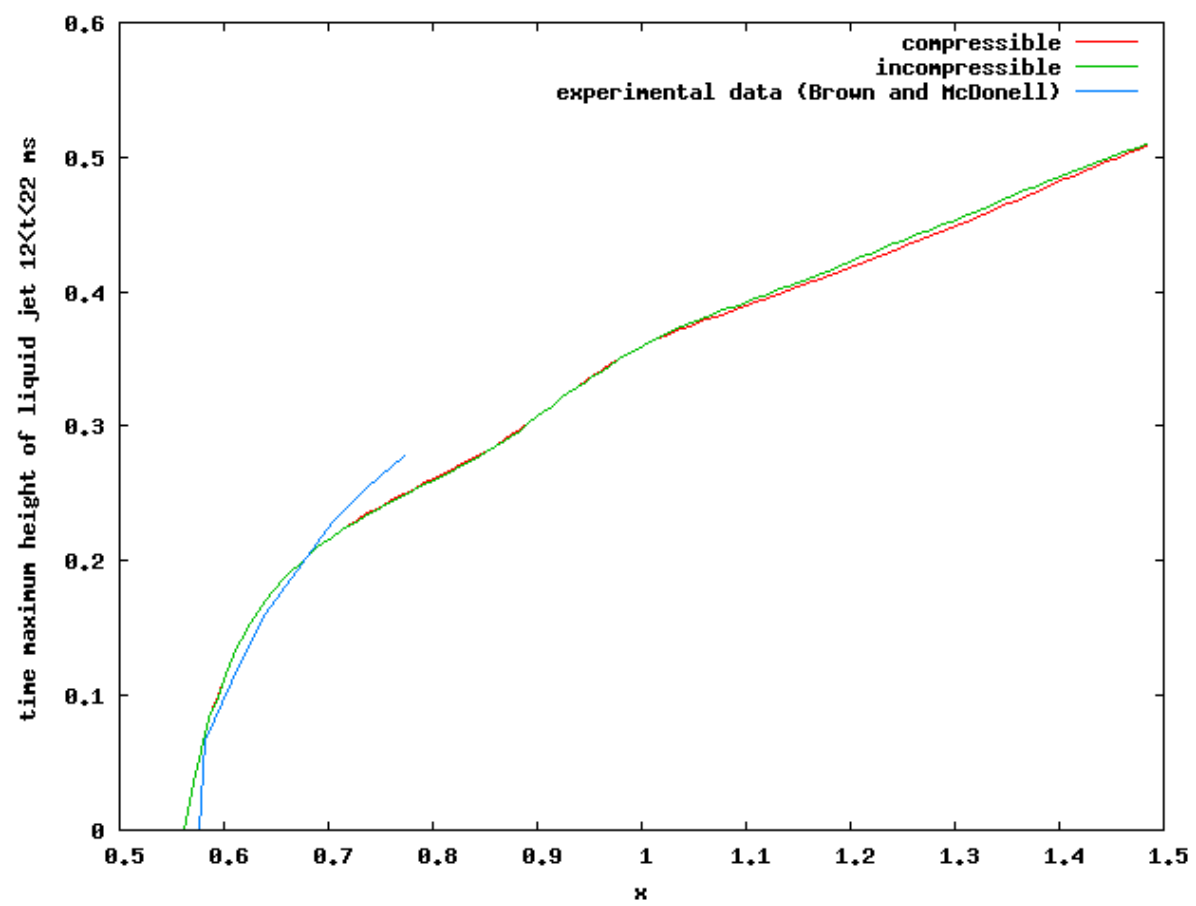

Figure 34: Time maximum height for the liquid jet versus the streamwise coordinate $x(y=0)$ in the laminar jet in a crossflow simulation. The compressible simulation is shown in red, incompressible simulation in green, and experimental data from Brown and McDonell [10] in blue. 


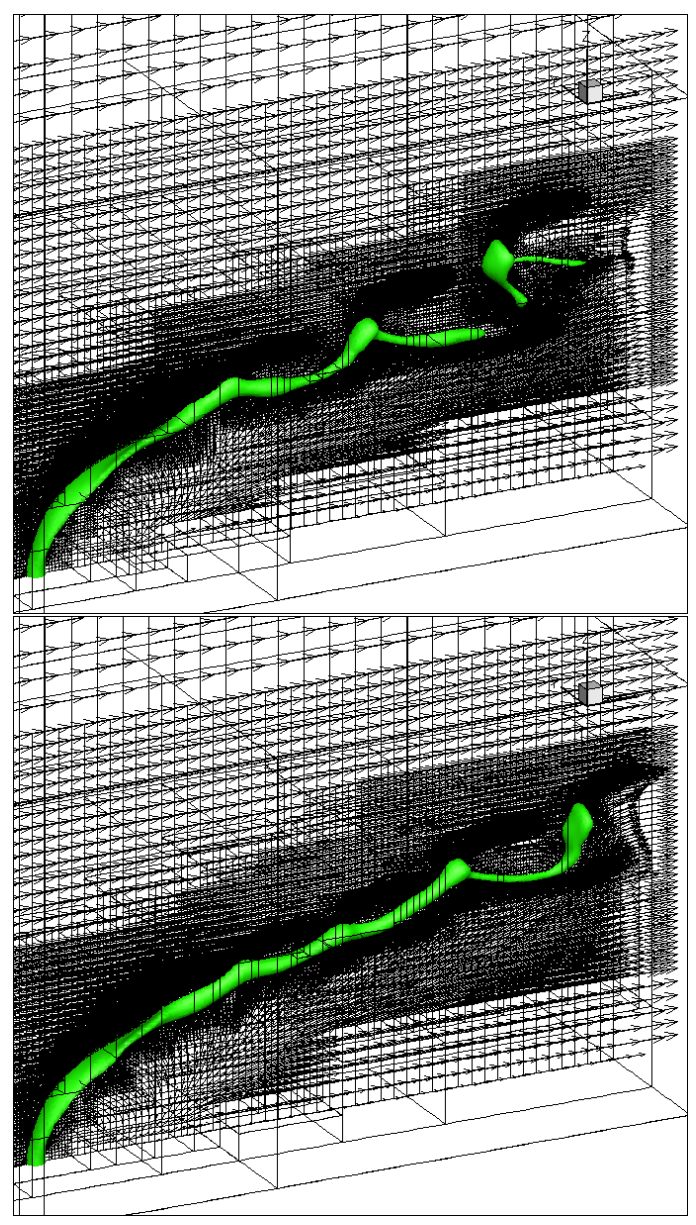

Figure 35: Simulation of liquid jet in gas cross flow. TOP: incompressible, $t=11.0 \mathrm{~ms}$; BOTTOM: compressible, $t=10.3 \mathrm{~ms}$ 


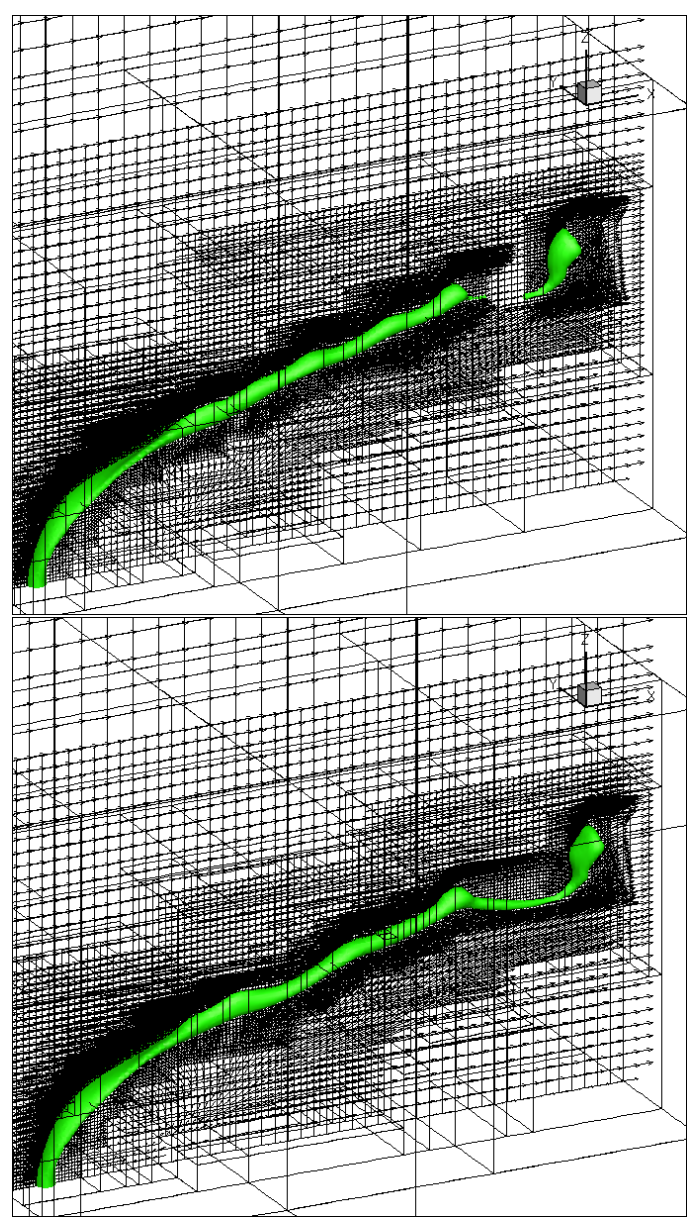

Figure 36: Simulation of liquid jet in gas cross flow. TOP: incompressible, $t=18.3 \mathrm{~ms}$; BOTTOM: compressible, $t=17.6 \mathrm{~ms}$ 


\section{Conclusions and Future Work}

A unified method for computing multiphase flows using conservative SemiLagrangian advection has been presented. The technique is applicable to both high- and low-Mach number flows. The Moment-of-Fluid method is used to reconstruct the interface, using a piecewise linear approximation in cut cells. The moment of fluid method has the ability to accurately capture change in topology and deformation of a material interface, which makes it well-suited to multiphase flow simulations. As with the semi-implicit method of $[38,21]$, no Riemann problem must be solved to capture the motion of material boundaries. Numerical experiments on standard 1D benchmark problems demonstrate that shock and interface speeds are accurately captured, as well as exhibiting less severe oscillation for problems with a large pressure jump across the interface. This method is robust enough to accurately capture the dynamics of a non-trivial two fluid simulation (e.g. oscillating water column). The method can be applied to materials with an exotic equation of state (e.g. JWL gas), with results that agree with ALE methods that explicitly track shock fronts and deform the underlying grid. Comparison of accuracy of the method in 2D cylindrical coordinates to 1D spherical coordinates is made, with agreement seen in shock magnitude and interface velocity. Preservation of symmetry is observed for the Spherical Explosion shock in 2D. We have demonstrated the asymptotically preserving property of our method by comparing our compressible multimaterial algorithm to the incompressible analogue for the problems of a 3D laminar jet in a cross-flow and the rise of a 3D axisymmetric gas bubble in liquid. Our method conserves mass of each material to machine accuracy. We find that this property, in the incompressible limit, automatically leads to volume conservation of each material.

The two key components of our algorithm, (1) Cell Integrated SemiLagrangian (CISL) advection for material moments, volume fractions, mass, momentum and energy, and (2) semi-implicit pressure correction, are both generalizable to unstructured grids. For ship-wave problems for example[27, 53], it is preferable to have a high aspect ratio grid that is body-fitted to the ship, whereas the deforming liquid and gas regions are allowed to cut the grid. Additionally, techniques to capture contact line dynamics and resolve sub-gridcell filaments are being developed in conjunction with the CISL scheme presented. 


\section{References}

[1] M. Aanjaneya, S. Patkar, and R. Fedkiw. A mass tracking formulation for bubbles in incompressible flow. J. Comput. Phys., 2013.

[2] H. Ahn and M. Shashkov. Multi-material interface reconstruction on generalized polyhedral meshes. J. Comput. Phys., 226(2):2096-2132, 2007.

[3] H. Ahn and M. Shashkov. Adaptive moment-of-fluid method. J. Comput. Phys., 228(8):2792-2821, 2009.

[4] M. Arienti and M. Soteriou. Time-resolved proper orthogonal decomposition of liquid jet dynamics. Phys. Fluids, 21, 2009.

[5] M. Arienti and M. Sussman. An embedded level set method for sharpinterface multiphase simulations of diesel injectors. International Journal of Multiphase Flow, 59:1-14, 2014.

[6] K. Arun, S. Noelle, M. Lukacova, and C.-D. Munz. An asymptotic preserving all mach number scheme for the euler equations of gas dynamics. Technical Report Bericht Nr. 348, RWTH Aachen University, Germany, October 2012.

[7] E. Aulisa, S. Manservisi, R. Scardovelli, and S. Zaleski. Interface reconstruction with least-squares fit and split advection in three-dimensional cartesian geometry. Journal of Computational Physics, 225(2):2301 2319, 2007.

[8] D. J. Benson. Volume of fluid interface reconstruction methods for multimaterial problems. App. Mech. Rev., 55:151-165, 2006.

[9] W. Bo, X. Liu, J. Glimm, and X. Li. A robust front tracking method: Verification and application to simulation of the primary breakup of a liquid jet. SIAM J. Sci. Comput., 33:1505-1524, 2011.

[10] C. T. Brown and V. G. McDonell. Near field behavior of a liquid jet in a crossflow. In Proceedings of the 19th Annual Conference on Liquid Atomization and Spray Systems, ILASS, May 2006. Toronto, Canada. 
[11] C.-H. Chang, X. Deng, and T. G. Theofanous. Direct numerical simulation of interfacial instabilities: A consistent, conservative, all-speed, sharp-interface method. J. Comput. Phys., 242:946-990, 2013.

[12] J. Cheng and C.-W. Shu. Positivity-preserving lagrangian scheme for multi-material compressible flow. Journal of Computational Physics, 257, Part A(0):143 - 168, 2014.

[13] A. J. Chorin. Numerical solution of the Navier-Stokes equations. Math. Comput., 22:745-762, October 1968.

[14] P. Degond, H. Liu, D. Savelief, and M.-H. Vignal. Numerical approximation of the euler-poisson-boltzmann model in the quasineutral limit. J. Sci. Comput., 51:59-86, 2012.

[15] A. Duffy, A. Kuhnle, and M. Sussman. An improved variable density pressure projection solver for adaptive meshes. unpublished. http://www.math.fsu.edu/sussman/MGAMR.pdf, 2012.

[16] V. Dyadechko and M. Shashkov. Reconstruction of multi-material interfaces from moment data. J. Comput. Phys., 227:5361-5384, 2008.

[17] C. Farhat, J.-F. Gerbeau, and A. Rallu. FIVER: A Finite Volume Method Based on Exact Two-Phase Riemann Problems and Sparse Grids for Multi-Material Flows with Large Density Jumps. J. Comput. Phys., 231:6360-6379, 2012.

[18] R. P. Fedkiw, T. Aslam, B. Merriman, and S. Osher. A non-oscillatory eulerian approach to interfaces in multimaterial flows (the ghost fluid method). J. Comput. Phys., 152:457-492, 1999.

[19] S. Galera, J. Breil, and P. Maire. A 2d unstructured multi-material cellcentered arbitrary lagrangian-eulerian (ccale) scheme using mof interface reconstruction. Comp. Fluids, 46:237-244, 2011.

[20] S. Galera, P. Maire, and J. Breil. A two-dimensional unstructured cellcentered multi-material ale scheme using vof interface reconstruction. $J$. Comput. Phys., 229:5755-5787, 2010.

[21] J. Gretarsson and R. Fedkiw. Fully conservative leak-proof treatment of thin solid structures immersed in compressible fluids. J. Comput. Phys., 245:160-204, 2013. 
[22] J. T. Gretarsson, N. Kwatra, and R. Fedkiw. Numerically stable fluidstructure interactions between compressible flow and solid structures. $J$. Comput. Phys., 230:3062-3084, 2011.

[23] F. Harlow and A. Amsden. Numerical calculation of almost incompressible flow. J. Comput. Phys., 3(1):80-93, 1968.

[24] A. Harten, J. M. Hyman, and P. D. Lax. On finite-difference approximations and entropy conditions for shocks. Communications on Pure and Applied Mathematics, XXIX:297-322, 1976.

[25] R. N. Hill and M. Shashkov. The symmetric moment-of-fluid interface reconstruction algorithm. J. Comput. Phys., 249:180-184, 2013.

[26] J. Hnat and J. Buckmaster. Spherical cap bubbles and skirt formation. Physics of Fluids, 19 (2):182-194, 1976.

[27] J. Huang, P. M. Carrica, and F. Stern. Coupled ghost fluid/two-phase level set method for curvilinear body-fitted grids. International Journal for Numerical Methods in Fluids, 55(9):867-897, 2007.

[28] S. Ii and F.Xiao. Cip/multi-moment finite volume method for euler equations: A semi-lagrangian characteristic formulation. J. Comp. Phys., 222:849-871, 2007.

[29] M. Jemison, E. Loch, M. Sussman, M. Shashkov, M. Arienti, M. Ohta, and Y. Wang. A coupled level set-moment of fluid method for incompressible two-phase flows. J. Sci. Comput., 54(2-3):454-491, Feb. 2013.

[30] E. Johnsen and T. Colonius. Shock-induced collapse of a gas bubble in shockwave lithotripsy. J. Acoust. Soc. Am., 124:2011-2020, 2008.

[31] S. Kadioglu and M. Sussman. Adaptive solution techniques for simulating underwater explosions and implosions. Journal of Computational Physics, 227:2083-2104, 2008.

[32] S. Kadioglu, M. Sussman, S. Osher, J. Wright, and M. Kang. A second order primitive preconditioner for solving all speed multi-phase flows. Journal of Computational Physics, 209(2):477-503, 2005.

[33] S. Y. Kadioglu. All speed multi-phase flow solvers. PhD Thesis, Florida State University, 2005. 
[34] M. Kang, R. Fedkiw, and X.-D. Liu. A boundary condition capturing method for multiphase incompressible flow. J. Sci. Comput., 15:323-360, 2000 .

[35] B. Koren, M. R. Lewis, E. H. van Brummelen, and B. van Leer. Riemann-problem and level-set approaches for homentropic two-fluid flow computations. J. Comput. Phys., 181:654-674, 2002.

[36] N. Kwatra, J. Su, J. T. Grétarsson, and R. Fedkiw. A method for avoiding the acoustic time step restriction in compressible flow. J. Comput. Phys., 228:4146-4161, June 2009.

[37] P. D. Lax. Hyperbolic systems of conservation laws and the mathematical theory of shock waves. In SIAM Regional Conference Series in Applied Mathematics, 1973.

[38] Lentine, J. Gretarsson, and R. Fedkiw. An unconditionally stable fully conservative semi-lagrangian method. J. Comp. Pyhs., 230(8):2857$2879,2011$.

[39] T. G. Liu, B. C. Khoo, and C. W. Wang. The ghost fluid method for compressible gas-water simulation. J. Comput. Phys., 204(1):193-221, Mar. 2005.

[40] R. C. Martineau and R. A. Berry. The pressure-corrected ice finite element method for compressible flows on unstructured meshes. J. Comput. Phys., 198:659-685, 2004.

[41] R. Nourgaliev, T. Dinh, M.-S. Liou, and T. Theofanous. The characteristics-based matching method (cbm) for high-speed fluid-fluid flows. ASME Journal of Fluids Engineering, 126:586-604, 2004.

[42] A. Phillipp, M. Delius, C. Scheffczyk, A. Vogel, and W. Lauterborn. Interaction of lithotripter-generated shock waves with air bubbles. $J$. Acoust. Soc. Am., 93:2496-2509, 1993.

[43] T. Ressguier, L. Signor, A. Dragon, and G. Roy. Dynamic fragmentation of laser shock-melted tin: experiment and modelling. International Journal of Fracture, 163(1-2):109-119, 2010. 
[44] R. Saurel and R. Abgrall. A simple method for compressible multifluid flows. SIAM J. Sci. Comput., 21:1115-1145, 1999.

[45] R. Scardovelli and S. Zaleski. Interface reconstruction with least-square fit and split eulerian-lagrangian advection. Int. J. Numer. Meth. Fluids, 41:251-274, 2003.

[46] S. Singh, J.-K. Choi, and G. Chahine. Optimum configuration of an expanding-contracting-nozzle for thrust enhancement by bubble injection. J. Fluids Eng., 134, 2012.

[47] M. Sussman. A parallelized, adaptive algorithm for multiphase flows in general geometries. Computers and Structures, 83:435-444, 2005.

[48] M. Sussman, A. Almgren, J. Bell, P. Colella, L. Howell, and M. Welcome. An adaptive level set approach for incompressible two-phase flows. $J$. Comput. Phys., 148:81-124, 1999.

[49] M. Sussman and M. Ohta. A stable and efficient method for treating surface tension in incompressible two-phase flow. SIAM J. Sci. Comput., 31(4):2447-2471, 2009.

[50] M. Sussman and E. Puckett. A coupled level set and volume of fluid method for computing 3D and axisymmetric incompressible two-phase flows. J. Comp. Phys., 162:301-337, 2000.

[51] T. Theofanous. Aerobreakup of newtonian and viscoelastic liquids. Annual Review of Fluid Mechanics, 43(1):661-690, 2011.

[52] Y. Wang, S. Simakhina, and M. Sussman. A hybrid level set-volume constraint method for incompressible two-phase flow. Journal of Computational Physics, 231(19):6438 - 6471, 2012.

[53] Z. Wang, J. Yang, and F. Stern. A new volume-of-fluid method with a constructed distance function on general structured grids. Journal of Computational Physics, 231(9):3703 - 3722, 2012.

[54] A. Wardlaw and H. Mair. Spherical solutions of an underwater explosion bubble. Shock and Vibration, 5:89-102, 1998.

[55] A. Wardlaw, Jr. Underwater explosion test cases. Technical Report IHTR 2069, ADB238684, Office of Naval Research, 1998. 
[56] P. Wesseling, D. van der Heul, and C. Vuik. Unified methods for computing compressible and incompressible flows. In Proceedings of the European Congress on computational methods in applied sciences and engineering, pages 1-23, Barcelona, Spain, 2000.

[57] G. Weymouth and D. K.-P. Yue. Conservative volume-of-fluid method for free-surface simulations on cartesian-grids. Journal of Computational Physics, 229(8):2853 - 2865, 2010.

[58] F. Xiao. Unified formulation for compressible and incompressible flows by using multi-integrated moments i: one-dimensional inviscid compressible flow. J. Comput. Phys., 195:629-654, 2004.

[59] F. Xiao, R. Akoh, and S. Ii. Unified formulation for compressible and incompressible flows by using multi-integrated moments ii: Multidimensional version for compressible and incompressible flows. J. Comput. Phys., 213:31-56, 2006.

[60] T. Yabe, Y. Ogata, K. Takizawa, T. Kawai, A. Segawa, and K. Sakurai. The next generation cip as a conservative semi-lagrangian solver for solid, liquid and gas. J. Comp. App. Math., 149:267-277, 2002.

[61] T. Yabe, F. Xiao, and T. Utsumi. The constrained interpolation profile method for multiphase analysis. Journal of Computational Physics, 169(2):556 - 593, 2001. 